\title{
Computational Modeling of High Temperature Gas-Grain Chemistry in Interstellar Medium (ISM)
}

\author{
Sean W. Schulte \\ Tucson, AZ
}

B.S. Chemistry, University of Arizona, 2014

A Thesis presented to the Graduate Faculty

of the University of Virginia in Candidacy for the Degree of

Master of Science.

Department of Chemistry

University of Virginia

December, 2017

\section{Committee:}

Dr. Eric Herbst (Advisor)

Dr. Robin T. Garrod 


\section{Table of Contents:}

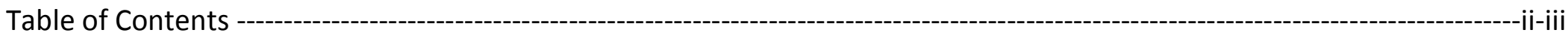

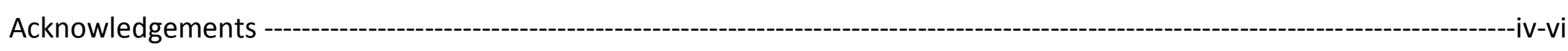

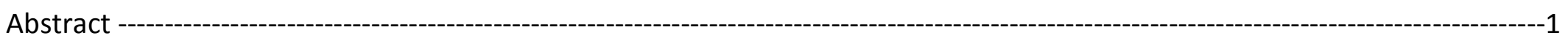

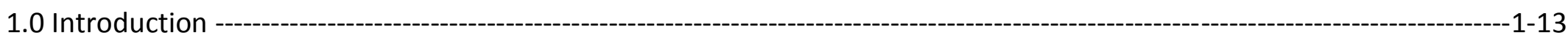

1.1 Chemistry on Bare Grains ---

1.2 Chemisorption and Physisorption ---

1.3 Reaction Mechanisms ---

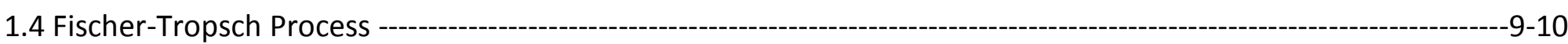

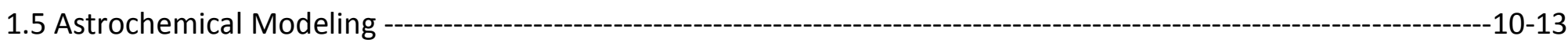

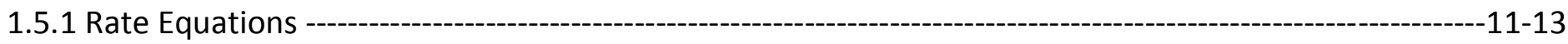

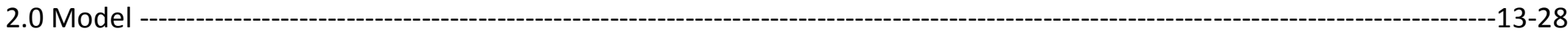

2.1 Gas-Phase Chemistry -

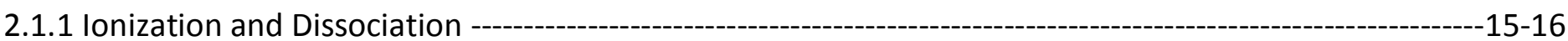

2.1.2 Treatment of UV and X-ray Photons ---

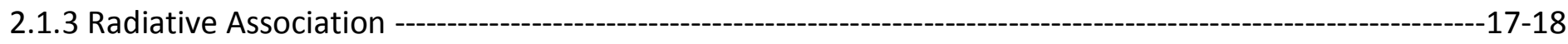

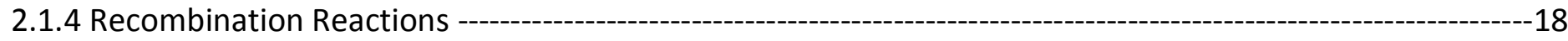

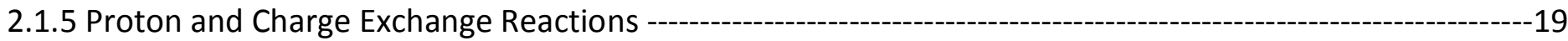

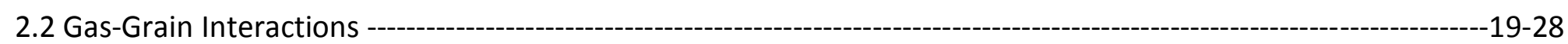

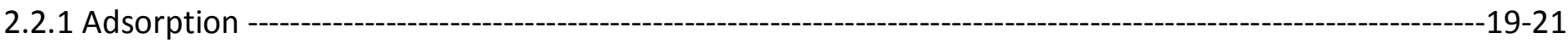




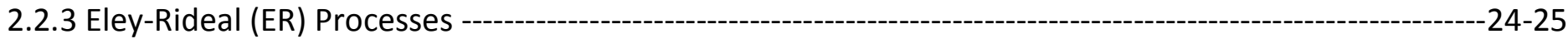

2.2.4 Dissociation Processes ---

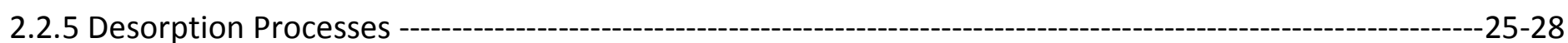

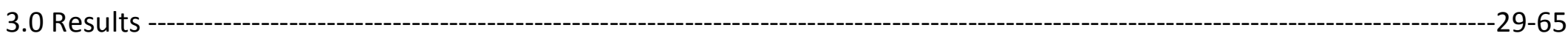

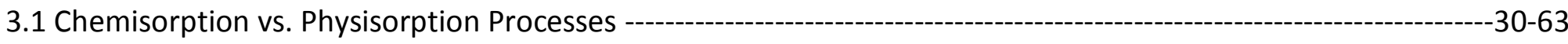

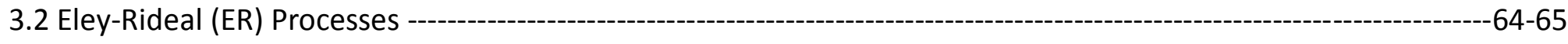

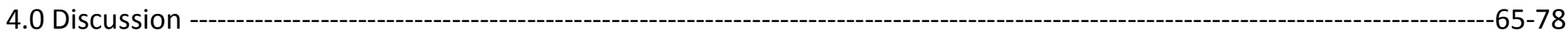

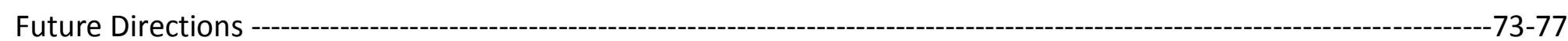

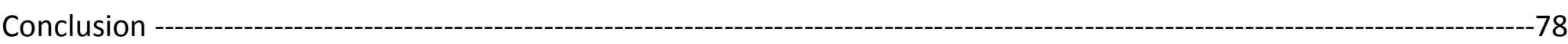

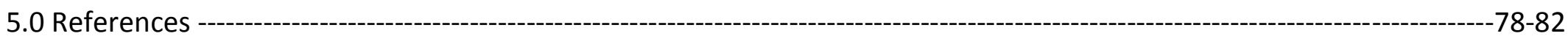

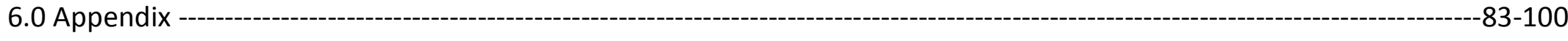

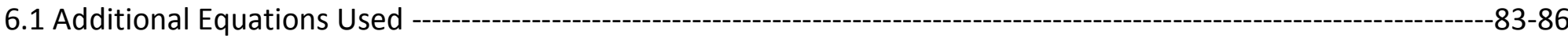

6.2 Additional Figures and Tables - 


\section{Acknowledgements:}

Throughout my education there's a lot of people who have helped me flourish. Not everyone to the same degree but hopefully I won't leave anyone out. To start I'd like to thank my advisor, Dr. Eric Herbst, for helping me throughout this process and doing his best to answer any questions I had throughout this journey. Without his vast knowledge of astronomy, chemistry and physics and his willingness to impart that knowledge I don't believe any of this would have been possible. I'd also like to thank my group members, both past and present, for all their support; Chris Shingledecker, Andrew Burkhardt, Dominique Maffuci, Ilsa Cooke, Roman Le Gal, Ugo Hincelin, Kinsuk Acharyya, and Ci Xue. I'd especially like to thank Chris Shingledecker who I shared an office with. He went out of his way to help me develop a better understanding of both astrochemistry and programming, and much like my advisor none of this would have been possible without his guidance. I'd also like to recognize Dr. Maxime Ruaud for all of his help. At the beginning of my research I was having difficulties getting my program to run, I went out to NASA Ames to visit Maxime for two weeks and he helped me to get the program running. I'll be forever grateful for Maxime's help. l'd like to thank my committee for all their guidance; Dr. Rob Garrod, Dr. Ian Harrison and Dr. Kateri Dubay. My committee is now composed of only Dr. Garrod and Dr. Herbst, when I decided to get an M.S. instead of a Ph.D. I reduced the size of my committee accordingly. Dr. Harrison was esp ecially helpful when it came to advising and Dr. Garrod has been quite helpful with many research related questions. I'd also like to thank 
the faculty and staff at University of Virginia (UVA) in general for being so cordial to me throughout my education, especially with regards to my disability.

As an undergraduate there were quite a few events that led me to graduate school at UVA. I'd be remiss if I didn't thank my undergraduate research advisor, Dr. Lucy Ziurys at The University of Arizona (U of A). It was in her research group that I got introduced to astrochemistry which set me on the path to UVA. I'd like to thank her group for all their support, especially Julie Anderson who I worked with on my project as an undergraduate. Julie was actually the one who recommended I apply at UVA and was very helpful with my undergraduate research project in which I explored the Deuterium to Hydrogen ratio in IRC+10216. I'd also like to thank the faculty and staff at $U$ of $A$ for being so helpful throughout my undergraduate career and being so willing to help me understand not only course material but help me plan for my future.

However, my experience in Dr. Ziurys group wasn't where I got my introduction to undergraduate research. In the summer of 2012, I was accepted to the program, Research Experience for Undergraduates (REU) at The University of Central Florida (UCF). I worked on modifying the surface of phase change nano-particles (nano-PCM's) in order to increase the heat transfer properties of nano-fluids. Although I decided to go in a different direction with my research I'd like to thank my advisor at UCF, Dr. Su, for all his help on my project. I'd also like to thank the graduate student I worked with on the project, Dr. Chaoming Wang. Chaoming was very 
helpful in showing me some lab fundamentals necessary to explore the melting point of nano-particles. He showed me how to use a Differential Scanning Calorimeter (DSC) and an LCR and also guided me in my literature searches.

Lastly, l'd like to thank my friends and family for all their support throughout my endeavors. Particularly my brothers, Kevin and Patrick Schulte and my niece Annabelle Schulte. My parents, Wim and Kathy Schulte, were as supportive as anyone could ask for and none of this would have been possible without their help. 


\begin{abstract}
:
We present a modified version of the Nautilus three-phase model in which high temperature gas-grain processes have been considered. 316 reactions have been added and 18 new species for a total of 11,800 reactions and 734 species. The modifications were necessary to account for the new gas-grain processes added where chemistry is dominated by species bound in chemisorption sites. A new thermal dissociation process was added which is believed to be the dominant dissociation process at high temperatures. In this model the mantle is considered chemically inactive since the grain is believed to be relatively bare in these temperature regimes. We focused on studying carbonaceous grains and assume the grain has catalytic properties similar to a graphite surface. Gas-grain formation routes were responsible for the gas phase abundances of methane, molecular hydrogen and the hydroxyl radical (from 200-400 K). A new dissociative adsorption mechanism is introduced for molecular hydrogen which provides an efficient destruction route at $\mathrm{T} \geq 500 \mathrm{~K}$ which results in a decrease in fractional abundance of up to two orders of magnitude when compared with the previous model. This reduction in molecular hydrogen results in the abundance of water being reduced when compared with previous models. An overall enhancement of atomic oxygen in the gas phase by almost an order of magnitude from $\mathrm{T} \geq 500 \mathrm{~K}$ is observed and alkanes are therefor destroyed more efficiently, especially at high temperatures.
\end{abstract}

Key Words: Astrochemistry, chemisorption, Fischer-Tropsch Process, heterogenous catalysis, carbonaceous grains.

\title{
1.0 Introduction:
}

Close to 200 molecules have been detected in the interstellar medium (ISM) to date. ${ }^{1}$ This is mostly through the application of rotational spectroscopy in the millimeter and submillimeter regime, although dust particles and molecules that lack a dipole moment can be probed by vibrational spectroscopy in the IR. Rotational spectroscopy can be done from high altitude observatories like Kitt Peak that are capable of looking in the $\mathrm{mm}$ regime. Space observatories or airborne observatories can look in the IR because they avoid atmospheric interferences. ${ }^{2}$ Early observations dealt with rather small molecules like the $\mathrm{OH}$ radical. In recent years 
complex organic molecules (COM's) have been detected. COM's are defined as molecules containing 6 or more atoms. Their precise formation mechanism is of great importance in astronomy and in a lot of cases is believed to take place on the grain surface. To help gain a better understanding of interstellar chemistry observational abundances can be augmented with theoretical studies as well as laboratory studies.

High temperature gas-grain chemistry is of great importance in gaining a more complete understanding of interstellar chemistry.$^{3-4}$ Although the effect of gas-surface reactions has been studied in industry and academia, to our knowledge it hasn't been explored in any great detail in astrophysical environments ${ }^{4}$ We will attempt to theoretically model high temperature-gas grain chemistry using a rate equation approach where chemisorption sites will be considered. High temperature grain chemistry is believed to be important in star forming regions like protoplanetary disk and hot cores. ${ }^{5}$ Gas phase abundances will eventually be compared to observational abundances in appropriate astrophysical environments. The rest of this thesis is organized as follows: Section one provides a brief background of astrochemistry and some important chemistry background that's pertinent to this thesis. In section two we describe the chemical model used and give a background of both gas phase chemistry and gas-grain chemistry. In section three we present the results for a molecular cloud run under standard conditions. In this section we compare the new high temperature gas-grain code with the Nautilus-three Phase Model presented in detail by Ruaud et al and look at the major effects of the new chemisorption processes. ${ }^{6}$ In section four we summarize the results and try to identify any shortcomings in this approach. 


\subsection{Chemistry on Bare Grains:}

ISM is composed of roughly $1 \%$ dust by mass. There are three main types of dust grains; silicate, carbonaceous and olivine grains. ${ }^{7}$ Dust grains are believed to have important catalytic properties that may aid in production of organic compounds in ISM, and may hold the answers to some unexplained phenomena in astrophysical environments..$^{4,-9}$ The formation of molecular hydrogen in interstellar medium was a long standing problem in astronomy. Formation was not believed to take place in the gas phase because there are no allowed transitions to the ground electronic state. Salpeter et al. found the most efficient route to be recombination of $\mathrm{H}$ atoms on the grain surface. ${ }^{10}$ This catalytic process gives high abundances of hydrogen in a time period two orders of magnitude shorter than the lifetime of the galaxy. ${ }^{11}$ Since these early studies of gas-grain chemistry the field has been expanded to explore the formation of many other organic compounds, particularly at low temperatures. ${ }^{12-15}$ 


\subsection{Chemisorption and Physisorption:}

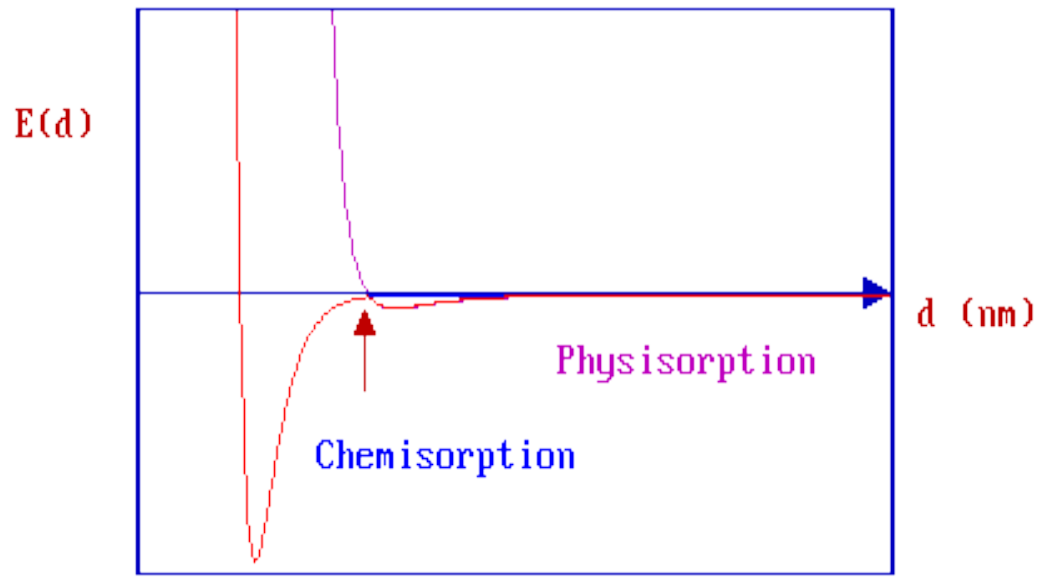

Figure 1.1: Chemisorption vs. physisorption potential energy (PE) curves. ${ }^{16}$

In the case of pure physisorption, the only attraction between the adsorbing species and the surface are weak van der Waals forces. When chemical bonding can occur the potential energy (PE) curve is dominated by a much deeper chemisorption well which results in much higher binding energies for the adsorbate ( $1 \mathrm{eV}$ vs $.1 \mathrm{eV})$. In Figure 1.1 above is a chemisorption well and on the right, is a physisorption well. When species accrete onto a surface at low temperatures they typically do so in a physisorption site. However, if the species possesses a little more thermal energy it can cross over into a 
chemisorption site. Often times a gas phase species will get trapped in a mobile pre-cursor state and will scan the surface to find a binding site. These states are dependent on the surface temperature and are short lived with a typical lifetime of 50 $\mu$ s. There's two distinct types of adsorption; molecular adsorption, where the adsorbing molecule stays intact during adsorption, and dissociative adsorption processes, where bonds break upon adsorption. ${ }^{17}$ In Figure 1.1 the arrow is pointing to what's known as the crossover point. If a diatomic comes into contact with the surface at the crossover point spontaneous dissociation will occur (dissociative adsorption). ${ }^{17-18}$ For this to occur the chemisorption site needs to be at a lower energy then the physisorption site and the barrier needs to lie below the energy of the incoming molecule. In this case dissociative adsorption will occur upon almost every collision. Figure 1.1 is representative of a diatomic molecule that would dissociatively adsorb upon contact with the surface, but the potential energy diagram may look different for different species and surfaces. At very high temperatures, species can accrete directly into a chemisorption site. A distinction can be made between the two radically different types of adsorption: chemisorption, where there is a direct chemical bond between the adsorbate and surface, and physisorption, where there is only weak van der waals forces holding the adsorbate to the surface. However, on a more fundamental level when an adsorbate is chemisorbed electrons are shared between the surface and adsorbate and as a result the electronic structure is significantly perturbed with respect to its gas phase equivalent.

Making this distinction is important because a strong or weak bond alone isn't enough to distinguish between chemisorption and physisorption. A species can be adsorbed in a shallow chemisorption well or conversely in a deep physisorption well. In 
this sense whether something is chemisorbed or physisorbed can be a matter of degree and this is an important distinction to make in the present work. ${ }^{17}$

Physisorption processes have already been treated at low temperatures, where atoms and molecules don't have sufficient energy to cross into a chemisorption well, so we'll be looking at high temperature grain reactions where chemisorption is believed to dominate. ${ }^{1,3,19}$ We'll be looking at gas phase temperatures of up to $800 \mathrm{~K}$, and grain temperatures equal to gas temperatures. This is expected to have implications on the way the chemistry is treated in each step as well as increased binding energies with respect to the physisorption values. ${ }^{3}$ We're expecting a change in fractional abundances that, when augmented with both observational data and our knowledge of astrophysical environments, will offer insight into the importance of these new processes. 


\subsection{Reaction Mechanisms:}

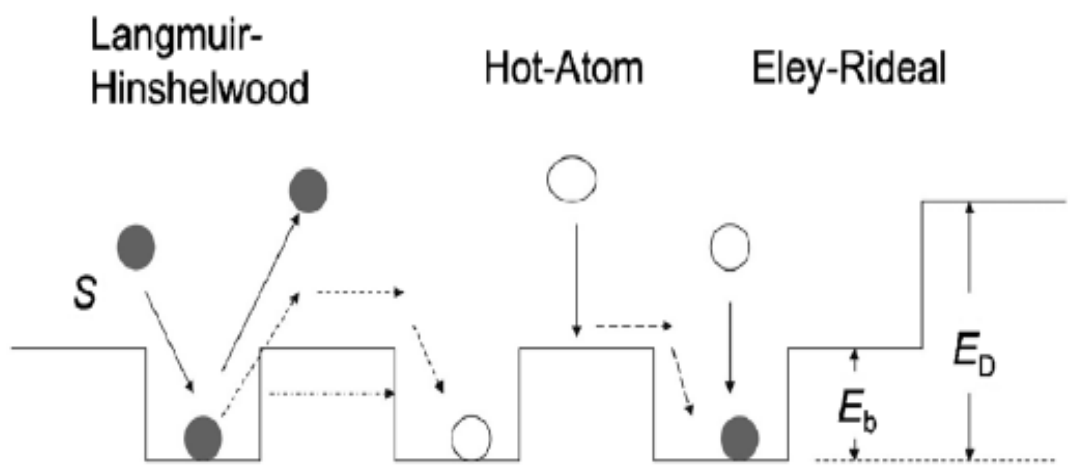

Figure 1.2: Three general types of surface reactions considered in surface science, Langmuir-Hinshelwood, Eley-Rideal and Hot-Atom mechanisms. ${ }^{1} \mathrm{~S}$ refers to the sticking coefficient, $\mathrm{E}_{\mathrm{h}}$ refers to the diffusion barrier and $\mathrm{E}_{\mathrm{D}}$ refers to the binding energy.

There are three general types of surface reactions: Langmuir-Hinshelwood (LH) mechanisms, Eley-Rideal (ER) mechanism and hot atom mechanisms (Figure 1.2 above). ${ }^{17}$ In a LH reaction mechanism species ' $A$ ' and ' $B$ ' thermally accrete onto the surface of the grain and then scan the surface to find a partner to react with. This is done classically (through hopping) or quantum mechanically (by tunneling under a barrier). ${ }^{20}$ Products can then desorb, either thermally or non-thermally.

In an ER reaction mechanism species ' $A$ ' accretes onto a grain surface and then reacts with an incoming ' $B$ ' species from the gas phase. Species ' $A$ ' is considered to be static on the grain in this mechanism. In this mechanism since the gas phase species ' $B$ ' reacts with an adsorbed species ' $A$ ', this reaction can be important in extreme conditions. One being when there's 
a high flux, and thus a high probability of ' $B$ ' finding ' $A$ ', the other when there's high surface coverage and a sufficient abundance of accreted material on the surface. ${ }^{21}$ In the current model we're using a diffusion to desorption barrier ratio of .2 in accordance with Gomer et al. ${ }^{22}$

In a hot atom mechanism species ' $A$ ' is accreted on the surface of the grain and species ' $\mathrm{B}$ ' collides with the grain. Species

' $B$ ' doesn't thermalize with the surface and moves some distance before reacting with an adsorbate. We focus on the LH and ER process in this model and don't consider contributions from the hot atom mechanism for the time being. 


\subsection{Fischer-Tropsch Process:}
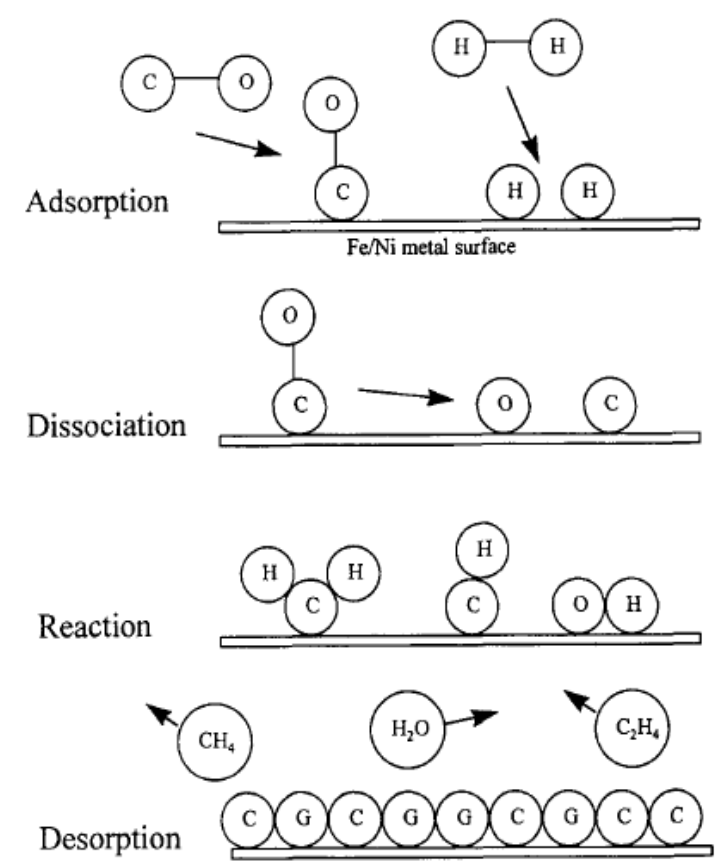

Figure 1.3: ${ }^{3}$ The reaction mechanism for Fe/Ni catalyzed conversion of $\mathrm{CO}$ to $\mathrm{CH}_{4}$. Follows a Langmuir-Hinshelwood reaction mechanism.

The first set of reactions considered is the Fischer-Tropsch process (Figure 1.3 above). Fischer-Tropsch catalysis is the family of surface reactions in which carbon monoxide $(\mathrm{CO})$ and molecular hydrogen $\left(\mathrm{H}_{2}\right)$ are converted to alkanes and alkenes using a catalyst. ${ }^{3,23}$ This catalyst is typically a transition metal such as iron or nickel but here the dust grain will act as 
the catalyst. ${ }^{4,8-9}$ This process is believed to be the most efficient way to convert CO into other forms of alkanes under high temperature regimes in astrophysical environments. ${ }^{3}$ Previous studies by Nuth et al. have found that when the surface of the grain becomes poisoned the surface acts as a better catalyst then the bare grain itself. Under normal circumstances decreasing the surface area of a catalyst will decrease the reaction rate but what they have found is by building up macromolecular grain coating the surface area of the catalyst essentially increases rather than being rendered unreactive. These sorts of complexities aren't taken into consideration in the current work. Although this process is presented as a rather simplistic Fischer-Tropsch process analogous to what's seen in industry in reality in these environment it's more of surfacemediated reactions since there is additional processes taking place. ${ }^{9}$ It's been suggested the Fischer-Tropsch process may help in not only explaining the organic make up of primitive meteorites, but may also imply protostellar nebulae may be rich in organic material.

\subsection{Astrochemical Modelling:}

One of the challenges in the field of astrochemistry is to try and understand the gas phase abundances of species observed in ISM. To attempt to resolve the chemical composition of astrophysical environments the synthetic mechanisms leading to products is studied. This is done by first constructing chemical models, which utilize large networks of chemical and physical processes to attempt to explain these abundances. Both experimental and theoretical work can be used to help 
constrain how these physical processes are treated. Of particular importance is how the rate coefficients are treated which help describe how these processes proceed. Model results are then compared with observational results to attempt to verify whether results are valid. ${ }^{24}$ Modelling the chemistry in astrophysical environments is normally done using one of the following techniques: 1) the rate equation approach, 2) the master equation approach, 3) kinetic Monte Carlo method. ${ }^{20-21,25-}$

${ }^{26}$ Each has its own advantages but in this research a rate equation approach will be utilized. Although this approach has the disadvantage of being less accurate then the master equation approach or Monte Carlo method it's computationally less expensive and is easier to couple with a network of gas phase and grain-surface reactions. The Monto Carlo method and master equation approach are better suited to handle smaller grains where stochastic fluctuations become important and small deviations in the grain surface begin to have a more significant impact on the chemistry. There is also something called a "modified rates approach" that is easier to couple with the rate equation approach and may help with dealing with smaller grains. ${ }^{13}$ This also won't be considered at this point.

\subsubsection{Rate Equations:}

The simplest model to implement and the one that is the focus of this research is the rate equation approach. In this method, each reaction in the network is assigned a rate coefficient which is usually temperature dependent. For a gas phase 
reaction between two neutral species or an ion and a neutral, the rate coefficient will follow a modified Arrhenius rate law as follows: ${ }^{24,27}$

(1.1) $\mathrm{k}(\mathrm{T})=\alpha *\left(\frac{\mathrm{T}}{300}\right)^{\beta} \exp \left(-\frac{\gamma}{\mathrm{T}}\right)$

$\alpha$ is the pre-exponential factor and has a slight temperature dependence, $\beta$ is the temperature dependent term and $\gamma$ is the activation barrier in Kelvin. These parameters are generally determined experimentally and sometimes it's necessary to extrapolate values outside the set temperature range, which leads to some uncertainty in determining the rate constant. ${ }^{24,28}$

We'll be using the chemical model Nautilus which has over 11,000 reactions and over 700 species. We've added 316 reactions and 18 species (see tables in appendix). The reactions are coupled to each other using the following two ordinary differential equations (ODE's) in the two-phase model: ${ }^{20}$

(1.2) $\frac{\mathrm{dn}_{\mathrm{i}}}{\mathrm{dt}}=\sum_{\mathrm{l}, \mathrm{m}} \mathrm{k}_{\mathrm{lm}} \mathrm{n}_{\mathrm{l}} \mathrm{n}_{\mathrm{m}}-\mathrm{n}_{\mathrm{i}} \sum_{\mathrm{i}=1} \mathrm{k}_{\mathrm{il}} \mathrm{n}_{\mathrm{l}}+\mathrm{k}_{\mathrm{i}}^{\mathrm{des}} \mathrm{n}_{\mathrm{i}}^{\mathrm{s}}-\mathrm{k}_{\mathrm{i}}^{\mathrm{acc}} \mathrm{n}_{\mathrm{i}}$

(1.3) $\frac{\mathrm{dn}_{\mathrm{i}}^{\mathrm{s}}}{\mathrm{dt}}=\sum_{\mathrm{l}, \mathrm{m}} \mathrm{k}_{\mathrm{lm}}^{\mathrm{s}} \mathrm{n}_{\mathrm{l}}^{\mathrm{s}} \mathrm{n}_{\mathrm{m}}^{\mathrm{s}}-\mathrm{n}_{\mathrm{i}}^{\mathrm{s}} \sum_{\mathrm{i}=1} \mathrm{k}_{\mathrm{il}}^{\mathrm{s}} \mathrm{n}_{\mathrm{l}}^{\mathrm{s}}-\mathrm{k}_{\mathrm{i}}^{\mathrm{des}} \mathrm{n}_{\mathrm{i}}^{\mathrm{s}}+\mathrm{k}_{\mathrm{i}}^{\mathrm{acc}} \mathrm{n}_{\mathrm{i}}$ 
These ODE's follow the formation and destruction pathways of each particular reaction. Here $\mathrm{k}_{\operatorname{lm}}^{\mathrm{s}}$ and $\mathrm{k}_{\mathrm{lm}}$ are, respectively, the surface and gas phase reaction rate constants, $\mathrm{k}_{\mathrm{i}}^{\text {acc }}$ and $\mathrm{k}_{\mathrm{i}}^{\mathrm{des}}$ are the accretion and desorption rate constants, and $\mathrm{n}_{\mathrm{i}}$ and $\mathrm{n}_{\mathrm{i}}^{\mathrm{s}}$ are the gas phase and surface concentration of species i. Nautilus uses DLSODES (Double Precision Livermore Solver for Ordinary Differential Equations with general sparse Jacobian matrix) solver to handle pseudo-time dependent chemical studies. ${ }^{29}$

\subsection{Model:}

The modifications made to the code were necessary to account for differences in how the adsorbate binds to the surface at high temperatures. ${ }^{17}$ Since we're dealing with chemisorption, where the adsorbate binds to the surface through a chemical bond, the chemistry will be slightly different then when ones dealing with physisorption. This is because for chemisorption the electronic structure of the adsorbate is significantly perturbed with respect to its gas phase equivalent. The modifications to the code will be elaborated on in the sections that follow, a more complete explanation of the gas-grain chemistry can be found in outside literature. ${ }^{6,20-21,27,29-30}$

\subsection{Gas-Phase Chemistry:}

Exothermic ion-neutral reactions involving non-polar neutral species typically follow the temperature independent Langevin rate coefficient $\mathrm{k}_{\mathrm{L}}$, as follows: ${ }^{24}$ 
(2.1) $\mathrm{k}_{\mathrm{L}}=2 \pi \mathrm{e} \sqrt{\frac{\alpha_{\mathrm{pol}}}{\mu}}$

where $\mathrm{e}$ is the electronic charge, $\alpha_{\mathrm{pol}}$ is the dipole polarizability, and $\mu$ is the reduced mass of reactants. The Langevin rate is how the rate coefficient of many ion-molecule reactions is modeled.

If the neutral is polar the situation is more complex. In this case the potential is anisotropic and depends on both the distance between reactants and the orientation of the permanent dipole, the long-range force is no longer central in nature.

${ }^{24}$ For the wide temperature range used here a method adopted from the trajectory scaling model of Su \& Chesnavich is used..$^{31}$ This approach is also described by Herbst \& Woon and is based on the following unitless parameter $\mathrm{x}:{ }^{32}$

(2.2) $x=\frac{\mu_{\mathrm{D}}}{\sqrt{2 \alpha_{\mathrm{pol}} \mathrm{k}_{\mathrm{B}} \mathrm{T}}}$

where $\mu_{\mathrm{D}}$ is the dipole moment of the reactant and $\mathrm{k}_{\mathrm{B}}$ is the Boltzmann constant. This approach also involves the following equations:

(2.3) $\frac{\mathrm{k}}{\mathrm{k}_{\mathrm{L}}}=.4767 \mathrm{x}+.6200(\mathrm{x} \geq 2)$

$(2.4) \frac{\mathrm{k}}{\mathrm{k}_{\mathrm{L}}}=\frac{(\mathrm{x}+.5090)^{2}}{10.526}+.9754(\mathrm{x}<2)$ 
As $\mathrm{x}$ approaches 0 , the rate coefficient approaches the Langevin rate. As $\mathrm{x}$ approaches infinity, the rate coefficient approaches a simple $\mathrm{T}^{-\frac{1}{2}}$ dependence. A complete list of polarizabilities and dipole moments needed for this approach has been calculated and reported by Herbst \& Woon. ${ }^{32}$ Equations 2.3 and 2.4 are total rate coefficients. If there's more than one production channel, then the rate coefficient has to be multiplied by the branching ratio. In Nautilus these equations appear as follows: ${ }^{33}$

$(2.5) \mathrm{k}=\alpha \beta\left(.62+.4767 \gamma\left(\frac{300}{\mathrm{~T}}\right)^{\frac{1}{2}}\right)$

(2.6) $\mathrm{k}=\alpha \beta\left(1+. .0967 \gamma\left(\frac{300}{\mathrm{~T}}\right)^{\frac{1}{2}}+\frac{\gamma^{2}}{10.526} * \frac{300}{\mathrm{~T}}\right)$

Here $\alpha, \beta$ and $\gamma$ are different then how they were defined earlier for the modified Arrhenius rate equation. $\alpha$ represent the branching ratio of the reaction. $\beta$ is the Langevin rate (2.1) and $\gamma$ represents the value of $x$ at $300 \mathrm{~K}$.

\subsubsection{Ionization and Dissociation:}

Ionization and dissociation rates can take place from either direct impact with cosmic ray (CR) particles, secondary UV photons induced by cosmic ray/ $\mathrm{H}_{2}$ interaction or interstellar FUV photons. ${ }^{29} 5$ 
Dissociation was treated in the following manner for cosmic ray ionization and dissociation: ${ }^{29}$

(2.7) $\mathrm{k}_{\mathrm{CR}}=\alpha \zeta_{\mathrm{CR}}$

$\alpha$ is the trial frequency, $\zeta_{\mathrm{CR}}$ is the cosmic ray ionization rate and was assumed to be $1.3 * 10^{-17} \mathrm{~s}^{-1}$.

(2.8) $\mathrm{k}_{\mathrm{FUV}}=\alpha * \exp \left(-\gamma * \mathrm{~A}_{\mathrm{V}}\right) * \chi$

Equation 2.8 treats the rate coefficient for photodissociation and ionization from interstellar FUV photons. $A_{v}$ is visual extinction, $\chi$ is unattenuated FUV flux, and $\exp \left(-\gamma * A_{V}\right)$ takes into account continuum attenuation from the dust. A more in-depth discussion of gas-phase reactions can be found in outside literature such as Wakelam et al. 2010. ${ }^{24,34}$

\subsubsection{Treatment of UV and X-ray Photons:}

The standard interstellar radiation field (ISRF) was used $(\chi=1.0)$. The self-shielding and cross-shielding involving the photodissociation of $\mathrm{H}_{2}$ are approximated using the method of Lee \& Herbst. ${ }^{35}$ This method is valid for enhancement of the radiation field, $\chi$, of up to 1000 . For $\mathrm{CO}$ self-shielding and cross-shielding the method of Visser et al. was adopted and coupled to the network. ${ }^{36}$

These regions can also be subjected to high fluxes of X-rays. X-rays can either ionize atoms directly and produce photo-electrons, or the photo-electrons can cause secondary ionization. ${ }^{34}$ In the current model the effect of X-rays is taken into account by varying the cosmic ray ionization rate. 


\subsubsection{Radiative Association:}

Radiative association reactions can occur between an ion and neutral or neutral and neutral, as follows: ${ }^{24}$

(2.9a) $\mathrm{A}^{+}+\mathrm{B} \rightarrow \mathrm{AB}^{+}+\mathrm{hv}$

(29b) $A+B \rightarrow A B+h v$

In either case, an activated complex forms first which can either lose sufficient energy by emitting a photon or redissociate from a sufficient amount of internal energy:

$(2.10 \mathrm{a}) \mathrm{A}^{+}+\mathrm{B} \rightarrow[\mathrm{AB}]^{+} \rightarrow \mathrm{AB}^{+}+\mathrm{hv}$

$(2.10 \mathrm{~b}) \mathrm{A}^{+}+\mathrm{B} \rightarrow[\mathrm{AB}]^{+} \rightarrow \mathrm{A}^{+}+\mathrm{B}$

To estimate the rate for radiative association the competition between emission and re-dissociation is considered:

(2.11) $\mathrm{k}_{\mathrm{RA}}=\mathrm{k}_{\mathrm{ass}}\left[\frac{\mathrm{k}_{\mathrm{rad}}}{\mathrm{k}_{\mathrm{rad}}+\mathrm{k}_{\mathrm{diss}}}\right]$

where $\mathrm{k}_{\mathrm{ass}}$ is the second-order rate coefficient for the formation of the complex, $\mathrm{k}_{\mathrm{rad}}$ is the first-order rate coefficient for the radiative stabilization of the complex and $\mathrm{k}_{\mathrm{diss}}$ is the first-order rate coefficient for re-dissociation of the complex.

Dissociation is more efficient for smaller systems; more atoms leads to more vibrations in the complex in which energy can 
be distributed which leads to slower re-dissociation. In interstellar environments radiative association is only a competitive process when the complex is large (>10 atoms) or one of the reactants is the relatively abundant molecular hydrogen, in such cases $\mathrm{k}_{\mathrm{diss}} \leq \mathrm{k}_{\mathrm{rad}}$ and radiative association occurs at the collisional rate.

\subsubsection{Recombination Reactions:}

There are two important types of recombination reactions in interstellar chemistry, radiative recombination (2.12a) and dissociative recombination $(2.12 \mathrm{~b}$ and $2.12 \mathrm{c}) \mathrm{:}^{24}$

$(2.12 \mathrm{a}) \mathrm{A}^{+}+\mathrm{e}^{-} \rightarrow \mathrm{A}+\mathrm{hv}$

(2.12b) $\mathrm{ABC}^{+} \rightarrow \mathrm{A}+\mathrm{B}+\mathrm{C}$

(2.12c) $\mathrm{ABC}^{+} \rightarrow \mathrm{AB}+\mathrm{C}$, etc

Radiative recombination reactions are typically important for an atomic ion recombining with an electron. Much like radiative association, in a radiative recombination reaction the product is stabilized by emission of a photon. The total rate constant for radiative recombination is typically $10^{-12}-10^{-13} \mathrm{~cm}^{3} \mathrm{~s}^{-1}$ with a $\mathrm{T}^{-1 / 2}$ temperature dependence. In dissociative recombination reactions a molecular ion recombines with a free electron and the energy released is dispersed through dissociation into one or more fragments. 


\subsubsection{Proton and Charge Exchange Reactions:}

Proton transfer reactions are important in many astrophysical environments. ${ }^{34}$ The exothermicities of the reaction place a limit on whether the reaction is likely to happen. At room temperature and above $\mathrm{H}_{2} \mathrm{O}, \mathrm{HCN}$ and $\mathrm{NH}_{3}$ are produced in greater abundance then in low temperature environments. Consider the following example:

(2.13a) $\mathrm{H}_{3} \mathrm{O}^{+}+\mathrm{HCN} \leftrightarrows \mathrm{HCNH}^{+}+\mathrm{H}_{2} \mathrm{O}$

This reaction is exothermic in the forward direction with an enthalpy change of $21.9 \mathrm{~kJ} \mathrm{~mol}^{-1} .{ }^{34}$ The backward reaction is slower but occurs at high temperatures like $800 \mathrm{~K}$. Charge transfer reactions are important in increasing the abundance of metallic ions. Unlike many other ion-molecule reactions the rate of charge transfer reactions doesn't go at the Langevin rate, their rate coefficient is typically modelled by a modified Arrhenius rate equation (1.1). A generic charge transfer reaction is shown here:

$(2.13 b) \mathrm{A}^{+}+\mathrm{B} \rightarrow \mathrm{B}^{+}+\mathrm{A}$

\subsection{Gas-Grain Interactions:} 2.2.1 Adsorption:

For adsorption, diffusion and thermal desorption the rate coefficients were computed following the work of Hasegawa et al. The rate for when a gas phase species adsorbs onto a grain is given as follows: ${ }^{20}$ 
(2.14) $\mathrm{R}_{\mathrm{ads}}(\mathrm{i})=\mathrm{S}(\theta) * \sigma_{\mathrm{d}} *\langle\mathrm{v}(\mathrm{i})\rangle * \mathrm{n}(\mathrm{i}) * \mathrm{n}_{\mathrm{d}}$

$\sigma_{d}$ is the cross section of the grain, $\langle v(i)\rangle$ is the thermal velocity of species $i, n(i)$ is the density of species $i, n_{d}$ is the number density of grains and $S(\theta)$ is the sticking coefficient, which is treated as a function of both temperature (both gas phase and grain) and surface coverage of the grain. Previous models of Nautilus have treated sticking in an over simplistic manner where neutral species were assigned a value of 1 and charged species a value of 0 . At high temperatures, the sticking coefficient being poorly constrained is even more problematic and will lead to errors. A value of 0 is still assigned to charged species but the sticking coefficient was treated as follows for gas phase temperatures above $100 \mathrm{~K}$, following the work of Hollenbach et al. ${ }^{37}$

(2.15) $\mathrm{S}(0)=\left[1+.04\left(\mathrm{~T}_{\text {gas }}+\mathrm{T}_{\text {grain }}\right)^{.5}+2 * 10^{-3} \mathrm{~T}_{\text {gas }}+8 * 10^{-6} \mathrm{~T}_{\text {gas }}^{2}\right]^{-1}$

For physisorption coverage is less important with regards to adsorption due to long range coupling and the adsorbate not needing a free site to adsorb to a surface. However, for chemisorption the adsorbate needs a free site to bind to due to electron sharing between the surface and adsorbate and short range interactions, if it hits a filled site the species is assumed to scatter. ${ }^{18}$ Equation 2.16 below was implemented to account for coverage dependence of the sticking coefficient:

(2.16) $\mathrm{S}(\theta)=\mathrm{S}(0) * \mathrm{P}_{\text {bare }}^{\mathrm{n}}(\theta)$ 
$\mathrm{S}(0)$ is defined above in equation 2.15 and here is the sticking coefficient at 0 monolayer coverage. The variance in the sticking probability as a function of both angle and energy isn't considered in this model and as such is still a very simplistic way to handle the sticking probability. $\mathrm{P}_{\text {bare }}^{\mathrm{n}}(\theta)$ is the probability that an incoming molecule hits a bare site and is defined as follows:

(2.17) $P_{\text {bare }}^{\mathrm{n}}(\theta)=(1-\theta)^{\mathrm{n}}$

$\theta$ is coverage and in this model, approaches 1 monolayer. ' $n$ ' is the number of sites needed to hold the adsorbate, for dissociative adsorption it's 2 and for molecular adsorption it's 1.

$\langle v(i)\rangle$ is the thermal velocity of species ' $i$ ' in the gas phase and is needed for the accretion reaction (equation 2.14 above): ${ }^{27}$

(2.18) $\left.\left.\langle\mathrm{v}(\mathrm{i})\rangle=\left(\sqrt{\left(8 \mathrm{k}_{\mathrm{B}} \mathrm{T} /\left(\pi \mu(\mathrm{i}) \mathrm{m}_{\mathrm{p}}\right.\right.}\right)\right)\right)$

where $T$ is the gas temperature, $k_{B}$ is Boltzmann's constant, $m_{p}$ is the protons mass and $\mu(i)$ is the reduced mass of the molecule 'i'. 


\subsubsection{Reaction/Diffusion:}

Most reactions considered here behave according to the Langmuir-Hinshelwood reaction mechanism where each reactant is pre-adsorbed to the surface of the grain before encountering another species to react with. ${ }^{17}$ In order to react the adsorbed species require mobility and the time scale to migrate from one site to another is modelled as follows: ${ }^{27,29}$

(2.19) $t_{\text {hop }}=v_{0}^{-1} \exp \left(-\frac{E_{\text {Diff }}}{T_{D}}\right)$

where $\mathrm{E}_{\mathrm{Diff}}$ is the diffusion barrier and $\mathrm{v}_{0}$ is the characteristic frequency. We adopt a value of $\mathrm{E}_{\mathrm{diff}}=.2 * \mathrm{E}_{\mathrm{des}}$, where $\mathrm{E}_{\mathrm{des}}$ is the desorption energy. This is in accordance with a review paper on diffusion by Gomer et al. ${ }^{22}$ For the characteristic frequency we used the harmonic oscillator relation: ${ }^{20}$

$(2.20) \mathrm{v}_{0}=\sqrt{2 * \frac{\mathrm{N}_{\mathrm{s}} \mathrm{E}_{\text {Des }}(\mathrm{i})}{\pi^{2} \mathrm{~m}(\mathrm{i})}}$

where $\mathrm{N}_{\mathrm{s}}$ is the number of surface sites on a $.1 \mu \mathrm{m}$ radius grain (assumed to be $10^{6}$ sites) and $\mathrm{m}(\mathrm{i})$ is the mass of the adsorbed particle.

The diffusion time required for an absorbed species to sweep over a number of sites equivalent to the grain surface is modeled as follows: 
(2.21) $t_{\text {diff }=} \mathrm{N}_{\mathrm{s}} \mathrm{t}_{\text {hop }}$

The diffusion rate, $R_{\text {diff, }}$, is defined as the inverse of $t_{\text {diff }}$. This is to define the surface reaction rate, $R_{i j}\left(\mathrm{~cm}^{-3} \mathrm{~s}^{-1}\right)$, occurring between a surface species $\mathrm{i}$ and $\mathrm{j}$ due to classical diffusion as follows:

(2.22) $R_{i j}=\kappa_{i j}\left(R_{d i f f, i}+R_{d i f f, j}\right) N_{i} N_{j} n_{d}$

where $N_{i}$ and $N_{j}$ are average number of species $i$ and $j$ on the grain, $n_{d}$ is the density of the grain and was set to $3 \mathrm{~g} \mathrm{~cm}^{-3}$. $\kappa_{\mathrm{ij}}$ is the probability for the reaction to occur and was treated as follows: ${ }^{38}$

(2.23) $\kappa_{\mathrm{ij}}=\frac{\alpha * \exp \left(-\frac{\mathrm{E}_{\mathrm{A}}}{\mathrm{T}_{\mathrm{D}}}\right)}{\alpha * \exp \left(-\frac{\mathrm{E}_{\mathrm{A}}}{\mathrm{T}_{\mathrm{D}}}\right)+\mathrm{t}_{\text {hop }}^{-1}(\mathrm{i})+\mathrm{t}_{\text {hop }}^{-1}(\mathrm{j})}$

where $\mathrm{E}_{\mathrm{A}}$ is activation energy. Equation 2.23 takes into consideration the competition between reaction and diffusion. An atom adsorbed on a grain surface can visit several sites before being released into the gas phase or recombining when it encounters another atom.

For light reactants like $\mathrm{H}$ and $\mathrm{H}_{2}$, tunneling effects can become important. In some cases it can be faster than the classical reaction, in which case tunneling replaces $\alpha * \exp \left(-\frac{E_{A}}{T_{D}}\right)$ in equation 2.23. Tunneling was treated in the following manner using a square potential: ${ }^{20}$ 
(2.24) $\mathrm{t}_{\mathrm{q}}=\mathrm{v}_{0}^{-1} \exp \left(\left(\frac{2 \mathrm{a}}{\hbar}\right) \sqrt{\mathrm{E}_{\mathrm{A}}}\right)$

where $\mathrm{v}_{0}$ is given by the harmonic oscillator approximation, ' $\mathrm{a}$ ' is the barrier thickness and was assumed to be $1 \AA$, and $\mathrm{E}_{\mathrm{A}}$ is activation energy. In the case of chemisorption processes, activation energies were taken from literature by assuming a carbonaceous grain behaves similar to a graphite surface. An Evans-Polanyi relationship can also be used to estimate activation energy and when a literature value wasn't available this approach was adopted (see appendix).

\subsubsection{Eley-Rideal (ER) Processes:}

Eley-Rideal (ER) processes were also considered, where an incident species ' $i$ ' reacts with an adsorbed species ' $j$ ' on the grain. The rate is treated as follows: ${ }^{21}$

(2.25) $\mathrm{R}_{\mathrm{ij}}=\mathrm{S}(0) * \eta_{\mathrm{j}} \sigma_{\mathrm{d}}\langle\mathrm{v}(\mathrm{i})\rangle \mathrm{n}(\mathrm{i}) \mathrm{n}_{\mathrm{d}}\left(\mathrm{cm}^{-3} \mathrm{~s}^{-1}\right)$

(2.26) $\eta_{\mathrm{j}}=\mathrm{n}_{\mathrm{s}}(\mathrm{j}) / \sum_{\mathrm{k}} \mathrm{n}_{\mathrm{s}}(\mathrm{k})$

Equation 2.26 represents the average density of the molecule on the surface. $\sigma_{d}$ is the cross section of the grain, $\langle v(i)\rangle$ is the thermal velocity of the incident species ' $i$ ', $n(i)$ its abundance and $n_{d}$ the number density of grains. $n_{s}(k)$ is the surface 
abundance of species ' $k$ '. For an ER reaction to take place the gas phase species needs to hit an adsorbate on the grain, or the species will scatter, hence equation 2.26. $\mathrm{S}(0)$ is the sticking coefficient at 0 coverage (equation 2.15).

\subsubsection{Dissociation Processes:}

Photodissociation processes were explained above in section 2.1.1. An additional thermal dissociation process has also been added to the model for chemisorption processes, as follows: ${ }^{18}$

(2.27) $\mathrm{k}_{\text {diss }}(\mathrm{i})=\mathrm{v}_{0}(\mathrm{i}) \exp \left(-\frac{\mathrm{E}_{\text {Diss }}(\mathrm{i})}{\mathrm{T}_{\text {grain }}}\right)$

where $v_{0}$ is the characteristic frequency. ${ }^{20} E_{\text {Diss }}(i)$ is the dissociation energy of species ' $i$ ', values used can be found in a table in the appendix. Dissociation energy is treated in a similar manner to activation energy, by assuming a carbonaceous grain behaves similar to a graphite surface. When a literature value isn't available dissociation energies can be estimated using the BOC-MP method (see appendix). ${ }^{17}$

\subsubsection{Desorption Processes:}

The thermal desorption rate is treated as follows using the Polanyi-Wigner equation: ${ }^{27}$

(2.28) $\mathrm{k}_{\mathrm{des}}(\mathrm{i})=\mathrm{v}_{0}(\mathrm{i}) \exp \left(-\frac{\mathrm{E}_{\text {Des }}(\mathrm{i})}{\mathrm{T}_{\text {grain }}}\right)$ 
where $\mathrm{v}_{0}$ is the characteristic frequency. ${ }^{20} \mathrm{E}_{\mathrm{Des}}(\mathrm{i})$ is the desorption energy of species ' $\mathrm{i}$ ', values used can be found in a table in the appendix. Desorption energies were taken from literature and to the best of my knowledge there doesn't appear to be any simplistic way to estimate them, just more rigorous quantum mechanical approaches like ab-initio calculation. For species that didn't have a literature value available, binding energy were treated as a free parameter under the assumption that their binding energies were in the order of magnitude of an eV, about ten times higher than the physisorption value. Some species such as homonuclear diatomics don't appear to chemisorb to a graphite surface, so the physisorption binding energy from previous models was used. ${ }^{39}$ Enthalpy of formation were estimated using Benziger's approach (see appendix). ${ }^{17}$

To treat reactive desorption the fraction ' $f$ ' of reactions resulting in desorption is calculated by considering the competition between the rate of desorption and the rate of energy lost to the grain: ${ }^{15}$

$$
\text { (2.29) } \mathrm{f}=\frac{\mathrm{aP}}{1+\mathrm{aP}}
$$

where $\mathrm{a}=v / v_{s}=1.0$ and is the ratio of surface molecule bond frequency to frequency at which energy is lost. Previous models of Nautilus use a value of .01, we use a value of 1.0 assuming reactive desorption is quite efficient at high temperatures on a bare grain in accordance with work by Dulieu et al. ${ }^{40} \mathrm{P}$ gives the probability of desorption and is based on the Rice-Rampsberger-Kessel theory and is described in more detail in Garrod 2007: 
(2.30) $P=\left[1-\frac{E_{\text {Des }}}{E_{\text {reac }}}\right]^{s-1}$

where $\mathrm{s}$ is the number of vibrational modes in the adsorbate-surface bond system and is 2 for a diatomic molecule and $3 \mathrm{~N}-5$

otherwise. $\mathrm{E}_{\mathrm{reac}}$ is the reaction exothermicity released during the reaction.

Using a value of 1.0 for ' $a$ ' in equation 2.29 is an oversimplification that will be discussed in more detail in the discussion section that follows. We chose this value because Dulieu et al. found reactive desorption to be much more efficient in producing $\mathrm{DO}_{2}$ on a bare graphite surface then on an icy mantle. ${ }^{40}$

A branching ratio was assumed where a fraction ' $f$ ' of the species desorbs into the gas phase, the energy from the reaction acts to break the surface-molecule bond. In the other case this energy is lost in lateral translation along the grain surface, and 1-f stays on the grain surface. By using a high value of 'a' in equation 2.29 this gives a branching ratio of roughly .5 for many species.

In these high temperature regimes desorption occurs mainly through the thermal desorption process or reactive desorption. However, photo-desorption is also considered. In recent years quantitative photo-desorption yields have been measured experimentally in labs. ${ }^{41-44}$ This process is quite important for icy mantles around protoplanetary disk where the temperatures are low enough for thermal desorption to be inefficient. However, in these high temperature regimes thermal desorption should be quite efficient. We still consider photo-desorption processes but rather than use the experimentally 
determined yields for icy mantles from previous work I'll be using a constant value, $\mathrm{Y}_{\mathrm{pd}}=10^{-4} \frac{\text { molecules }}{\text { photon }}$, in accordance with the work of Van Dischoeck et al. ${ }^{45}$ Photo-desorption rates are treated in the following manner:

(2.31) $\mathrm{k}_{\mathrm{des}, \mathrm{UV}}(\mathrm{i})=\frac{\mathrm{F}_{\mathrm{UV}} \mathrm{S}_{\mathrm{UV}} \exp \left(-2 \mathrm{~A}_{\mathrm{V}}\right) \mathrm{Y}_{\mathrm{pd}} \pi \mathrm{r}_{\mathrm{dust}}^{2}}{\mathrm{~N}_{\text {site }}\left[\mathrm{s}^{-1}\right]}$

(2.32) $\mathrm{k}_{\mathrm{des}, \mathrm{UV}-\mathrm{CR}}(\mathrm{i})=\frac{\mathrm{F}_{\mathrm{UV}-\mathrm{CR}} \mathrm{S}_{\mathrm{UV}-\mathrm{CR}} \exp \left(-2 \mathrm{~A}_{\mathrm{V}}\right) \mathrm{Y}_{\mathrm{pd}} \pi \mathrm{r}_{\mathrm{dust}}^{2}}{\mathrm{~N}_{\mathrm{site}}\left[\mathrm{s}^{-1}\right]}$

Equation 2.31 is how the rate for photo-desorption from standard interstellar UV-photons is calculated. Equation 2.32 is how the rate for the photo-desorption from secondary UV photons induced by cosmic rays is calculated. In these equation $\mathrm{S}$ is the scaling factor for the UV radiation field, $\mathrm{F}$ it's strength ( photons $\mathrm{cm}^{-2} \mathrm{~s}^{-1}$ ) and $\mathrm{r}_{\text {dust }}$ the grain radius. In this model we set $\mathrm{F}_{\mathrm{UV}}=1.0 * 10^{8}$ photons $\mathrm{cm}^{-2} \mathrm{~s}^{-1}$ and $\mathrm{F}_{\mathrm{UV}-\mathrm{CR}}=1.0 * 10^{4}$ photons $\mathrm{cm}^{-2} \mathrm{~s}^{-1} \cdot{ }^{44,46}$ The main difference between how photo-desorption is treated in these high temperature regimes is the scaling factor, we restrict desorption to the first monolayer of a bare grain rather than the first two monolayers for an ice mantle considered in previous models. ${ }^{6}$ This is a very inefficient process; the rate constant is $10-20$ orders of magnitude smaller than thermal desorption or reactive desorption. Photo-desorption is an indirect process which is driven by photo-absorption in sub-surface molecular layers and an energy transfer to the surface. ${ }^{47}$ Although this process has been studied on icy mantles it's importance on bare grains in these high temperature regimes isn't entirely clear. For this reason, it won't be discussed further. 


\subsection{Results:}

All of the work presented in this section was done using a modified version of the three-phase gas-grain model presented in detail by Ruaud et al. 6, 21 The third phase was turned off so mantle chemistry was not considered. The adopted gas phase network is kida.uva.2014. ${ }^{48}$ The grain network is the one originally presented in Ruaud et al. ${ }^{21}$ and was adopted from work by Garrod et al. ${ }^{15}$ To get a feeling for the fractional abundance as a function of time a pseudo-time dependent model was run at gas temperatures of up to $800 \mathrm{~K}$, grain temperatures are assumed to be equivalent to gas temperatures. The model was run under fixed and homogenous physical conditions. The fixed total hydrogen abundance was set to $2 * 10^{4} \mathrm{~cm}^{-3}$, The cosmic ray ionization rate was set to $1.3 * 10^{-17} \mathrm{~s}^{-1}$, For visual extinction a value of 10 is used, the standard interstellar radiation field was used. Initial abundances are presented in Table 3.1 below, low metal oxygen-rich abundances were used and the model is run starting from molecular hydrogen and every other species in its atomic form. Models with these physical conditions and parameters are termed "standard" ${ }^{34} 316$ reactions have been added to the network and 18 species; see Tables A1-A2 in the appendix. The final network has 734 species and over 11,800 reactions. 


\begin{tabular}{|c|c|}
\hline \multicolumn{2}{|c|}{ Table 3.1: Initial Fractional abundances with Respect to Total } \\
\hline \multicolumn{2}{|c|}{ Hydrogen: } \\
\hline Species: & Low metal value: \\
\hline $\mathrm{H}_{2}$ & .5 \\
\hline $\mathrm{He}$ & .14 \\
\hline $\mathrm{O}$ & $1.76(-4)$ \\
\hline $\mathrm{N}$ & $2.14(-5)$ \\
\hline $\mathrm{F}$ & $2.0(-8)$ \\
\hline $\mathrm{Cl}$ & $3.0(-9)$ \\
\hline $\mathrm{C}^{+}$ & $7.3(-5)$ \\
\hline $\mathrm{Fe}^{+}$ & $3.0(-9)$ \\
\hline $\mathrm{Mg}^{+}$ & $3.0(-9)$ \\
\hline $\mathrm{Na}^{+}$ & $3.0(-9)$ \\
\hline $\mathrm{P}^{+}$ & $3.0(-9)$ \\
\hline $\mathrm{S}^{+}$ & $2.0(-8)$ \\
\hline $\mathrm{Si}^{+}$ & $3.0(-9)$ \\
\hline
\end{tabular}

\subsection{Chemisorption vs. Physisorption Processes:}

In physisorption the adsorbate bonds to the grain surface through weak van-der-waals forces which are caused by mutually induced dipole moments in the electronic structure of the adsorbate and surface. ${ }^{18}$ These forces are very weak (binding energies of roughly $.1 \mathrm{eV}$ ) and dominate the chemistry in low temperature regimes. They are also responsible for adsorption of noble gases and other closed shell species $\left(\mathrm{CH}_{4}\right.$ or $\left.\mathrm{H}_{2}\right)$ even at higher temperatures. The surface must be kept at low enough temperatures (below liquid $\mathrm{N}_{2}$ temperature) to build up appreciable amounts of surface species. 
Furthermore, the electronic structure of the surface doesn't seem to play a significant role which means multilayer adsorption can occur.

The forces responsible for chemisorption are based soley on quantum mechanical interactions between the adatom and surface (overlap of respective wave functions). This leads to higher binding energies then is typically observed for physisorbed species, with binding energies in the order of magnitude of an eV.

In a series of papers from 2002-2005 Cazaux et al. looked at the formation of molecular hydrogen when taking into account the presence of both physisorbed and chemisorbed sites on the grain surface. ${ }^{49-51}$ What they found was two distinct regimes of $\mathrm{H}_{2}$ formation which reflect 2 different binding sites. At low temperatures ( $\mathrm{T} \leq 100 \mathrm{~K}$ ), physisorbed $\mathrm{H}$ species will sweep the surface until it finds another $\mathrm{H}$ species to recombined with. At very low temperatures ( $\mathrm{T} \leq 20 \mathrm{~K})$, this happens between two physisorbed hydrogen atoms. ${ }^{11,52}$ Between $20-100 \mathrm{~K}$ the physisorbed hydrogen atom sweeps the surface until it recombines with a chemisorbed hydrogen atom. ${ }^{49}$ At temperatures above $100 \mathrm{~K}$ recombination occurs between two chemisorbed species. In this model I only consider a few interaction between physisorbed sites and chemisorbed sites and assume species accrete directly into a chemisorption site. Several species won't chemisorb to the grain surface and I use their physisorbed binding energies $\left(\mathrm{BH}_{2}, \mathrm{BC}_{2}, \mathrm{BO}_{2}, \mathrm{BH}_{2} \mathrm{O}\right)$, in those cases the interaction between the physisorbed site and chemisorbed site is considered and those species are assumed to accrete into physisorbed sites. 
Diffusion between two chemisorbed sites or two physisorbed sites occurs horizontally, in our case we're using a diffusion to desorption barrier of .2. However, when considering diffusion between both a physisorbed site and chemisorbed site there's also a vertical component that should be considered, we're not considering that yet since only have a few of these reactions are in the network. ${ }^{25}$ Since we're not yet considering the interaction between chemisorbed and physisorbed binding sites in any great detail the focus is on temperatures above $200 \mathrm{~K}$, where chemisorption processes should dominate. However, clearly this is problematic since accretion directly into a chemisorption site does have a barrier (.2 eV for $\mathrm{H}$ for instance). ${ }^{25}$ This process is likely to take place at higher temperatures, but at $200 \mathrm{~K}$ the physisorption sites are likely to be entered first at which point diffusion to a chemisorption site will occur. This over simplification will be discussed in more detail in the discussion section. 


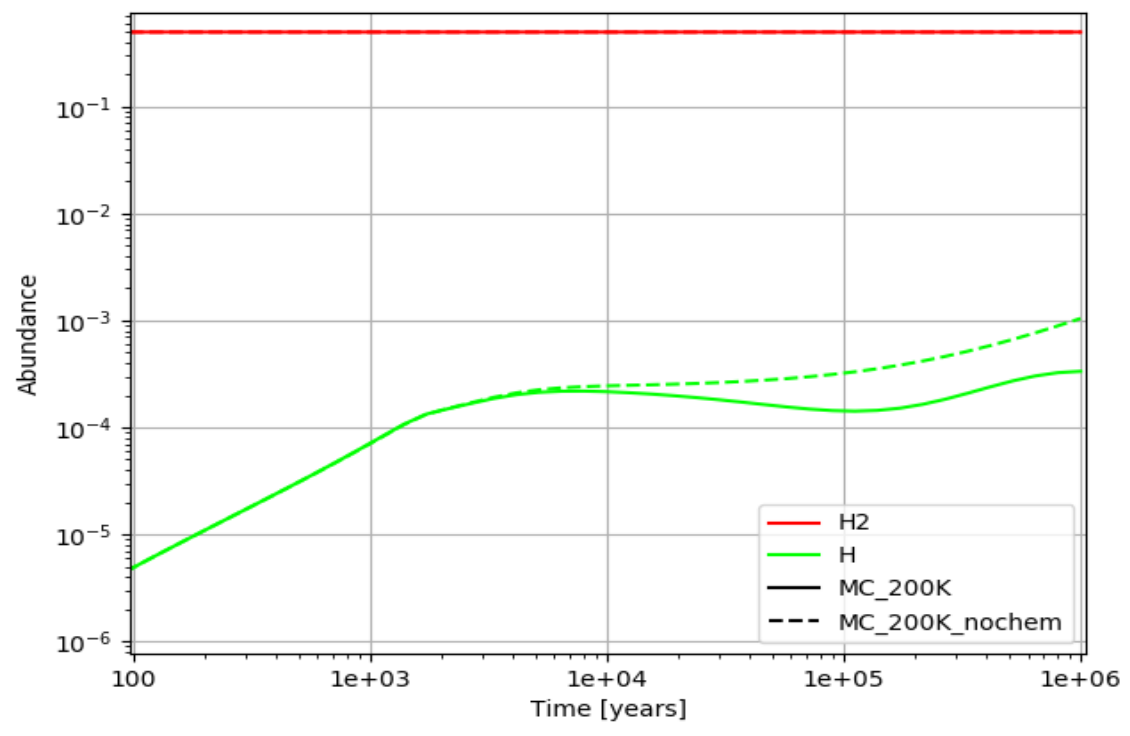

Figure 3.1: Fractional abundance of $\mathrm{H}$ and $\mathrm{H}_{2}$ with respect to $\mathrm{n}_{\mathrm{H}}$ as a function of time for a molecular cloud ran under standard conditions at 200 K. 


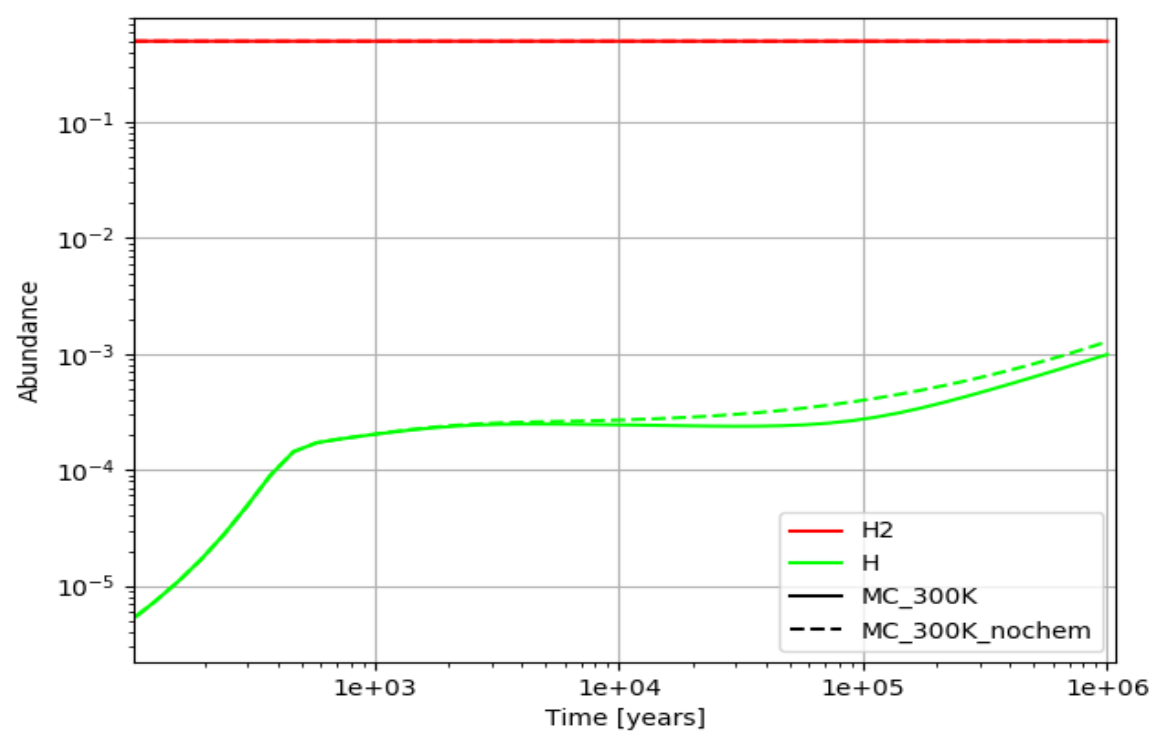

Figure 3.2: Fractional abundance of $\mathbf{H}$ and $\mathbf{H}_{2}$ with respect to $\mathbf{n}_{\mathbf{H}}$ as a function of time for a molecular cloud ran under standard conditions at 300 K. 


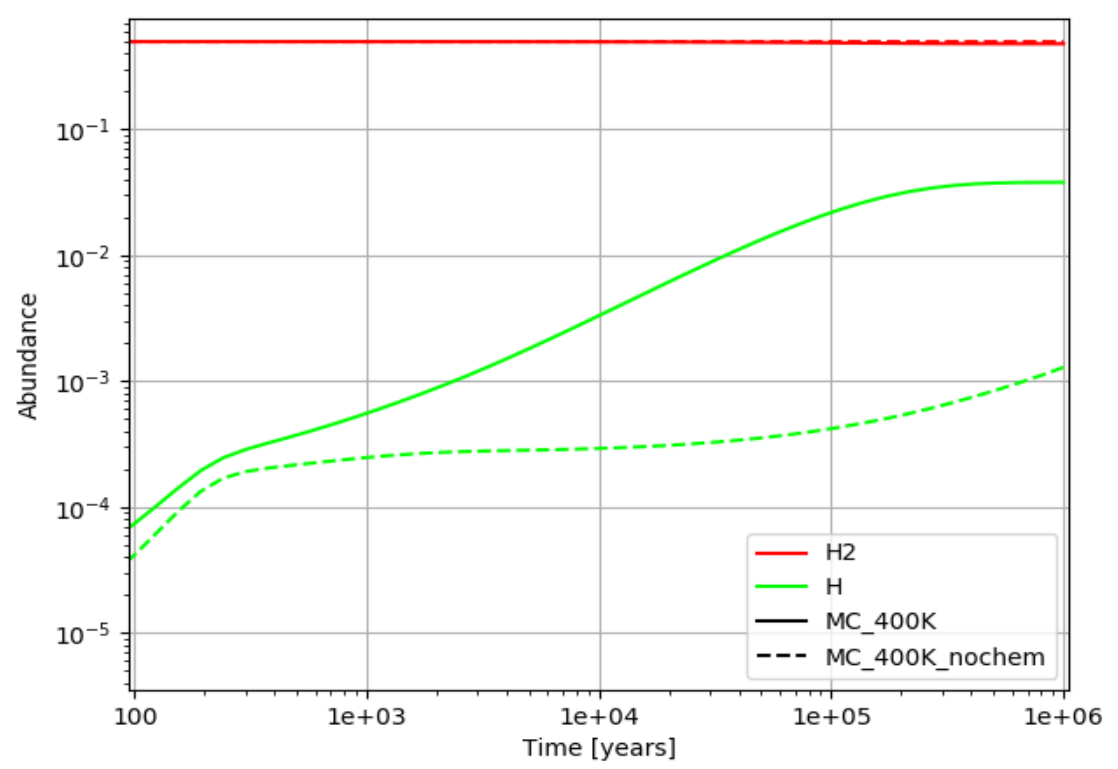

Figure 3.3: Fractional abundance of $\mathbf{H}$ and $\mathbf{H}_{2}$ with respect to $\mathbf{n}_{H}$ as a function of time for a molecular cloud ran under standard conditions at 400 K. 


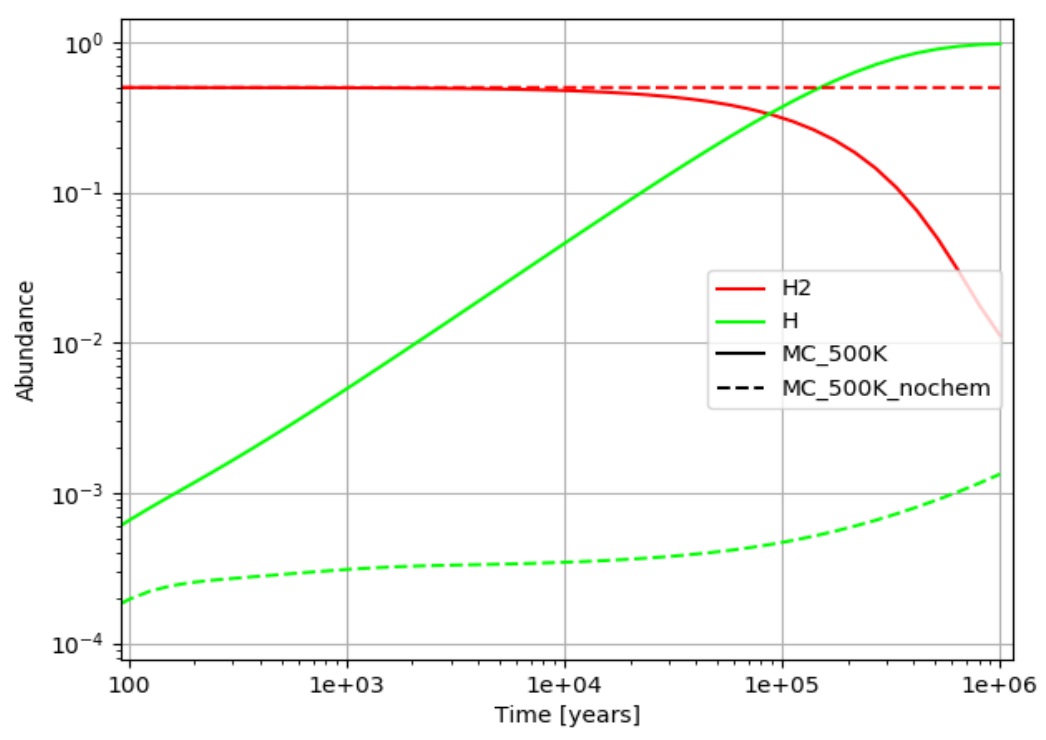

Figure 3.4: Fractional abundance of $\mathrm{H}$ and $\mathrm{H}_{2}$ with respect to $\mathbf{n}_{\mathrm{H}}$ as a function of time for a molecular cloud ran under standard conditions at 500 K. 


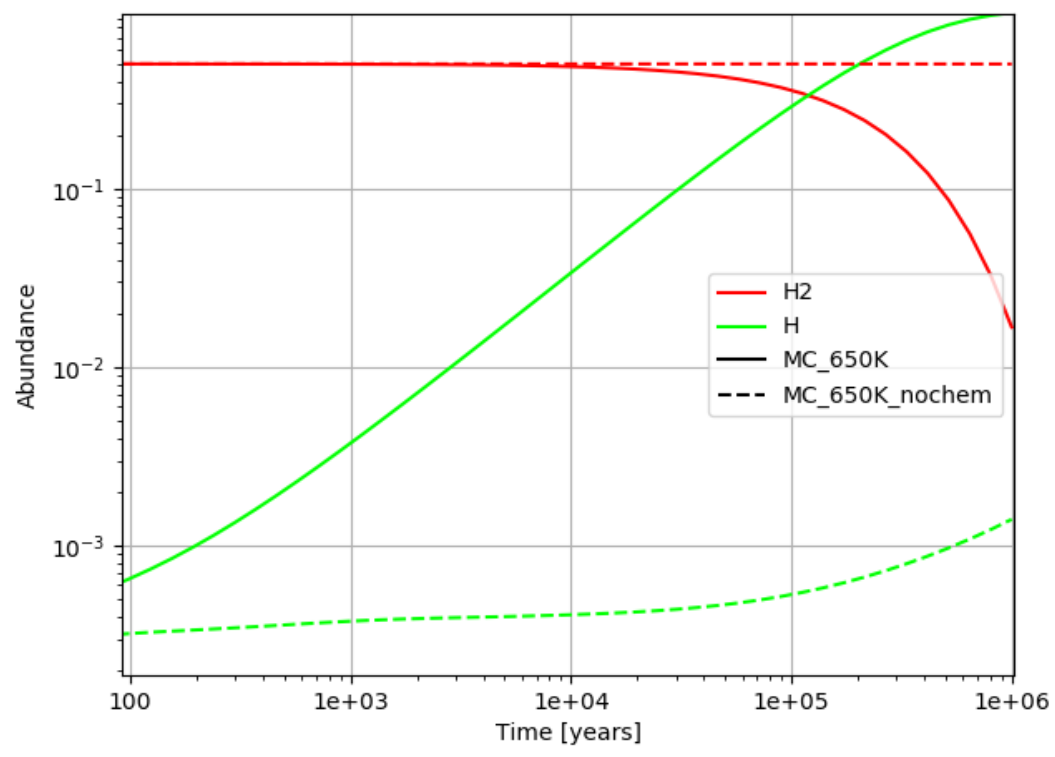

Figure 3.5: Fractional abundance of $H$ and $H_{2}$ with respect to $n_{H}$ as a function of time for a molecular cloud ran under standard conditions at 650 K. 


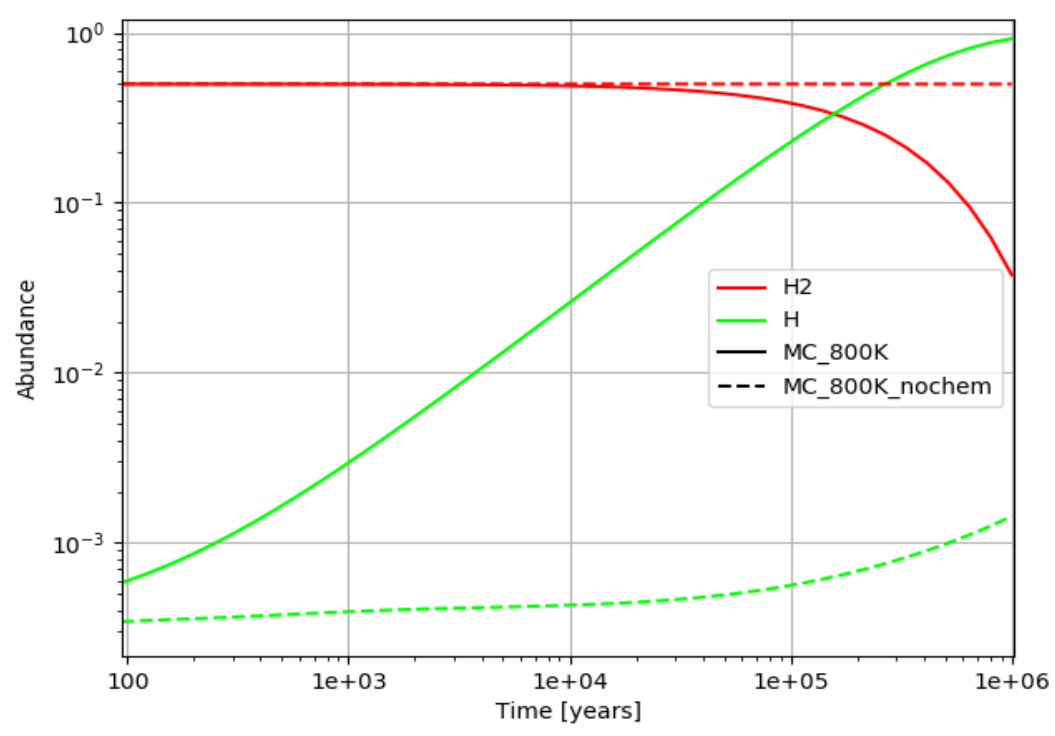

Figure 3.6: Fractional abundance of $\mathrm{H}$ and $\mathrm{H}_{2}$ with respect to $\mathbf{n}_{\mathrm{H}}$ as a function of time for a molecular cloud ran under standard conditions at 800 K. 


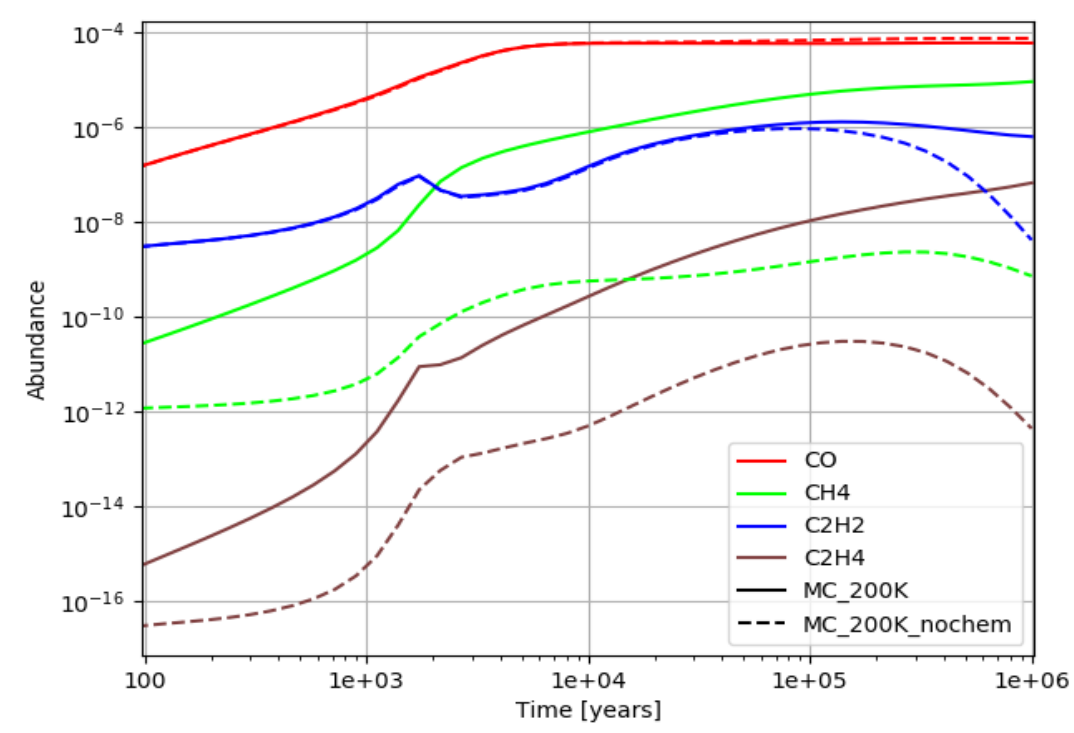

Figure 3.7: Fractional abundance of $\mathrm{CO}, \mathrm{CH}_{4}, \mathrm{C}_{2} \mathrm{H}_{2}$ and $\mathrm{C}_{2} \mathrm{H}_{4}$ with respect to $\mathrm{n}_{\mathrm{H}}$ as a function of time for a molecular cloud ran under standard conditions at $200 \mathrm{~K}$. 


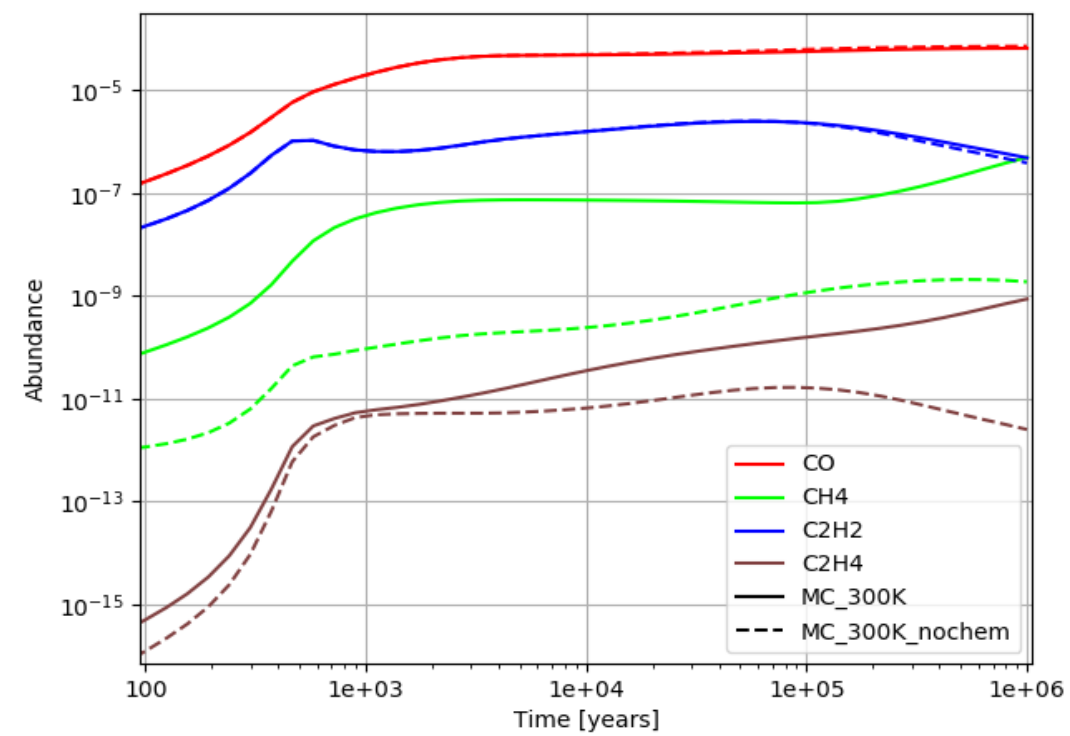

Figure 3.8: Fractional abundance of $\mathrm{CO}, \mathrm{CH}_{4}, \mathrm{C}_{2} \mathrm{H}_{2}$ and $\mathrm{C}_{2} \mathrm{H}_{4}$ with respect to $\mathrm{n}_{\mathrm{H}}$ as a function of time for a molecular cloud ran under standard conditions at $300 \mathrm{~K}$. 


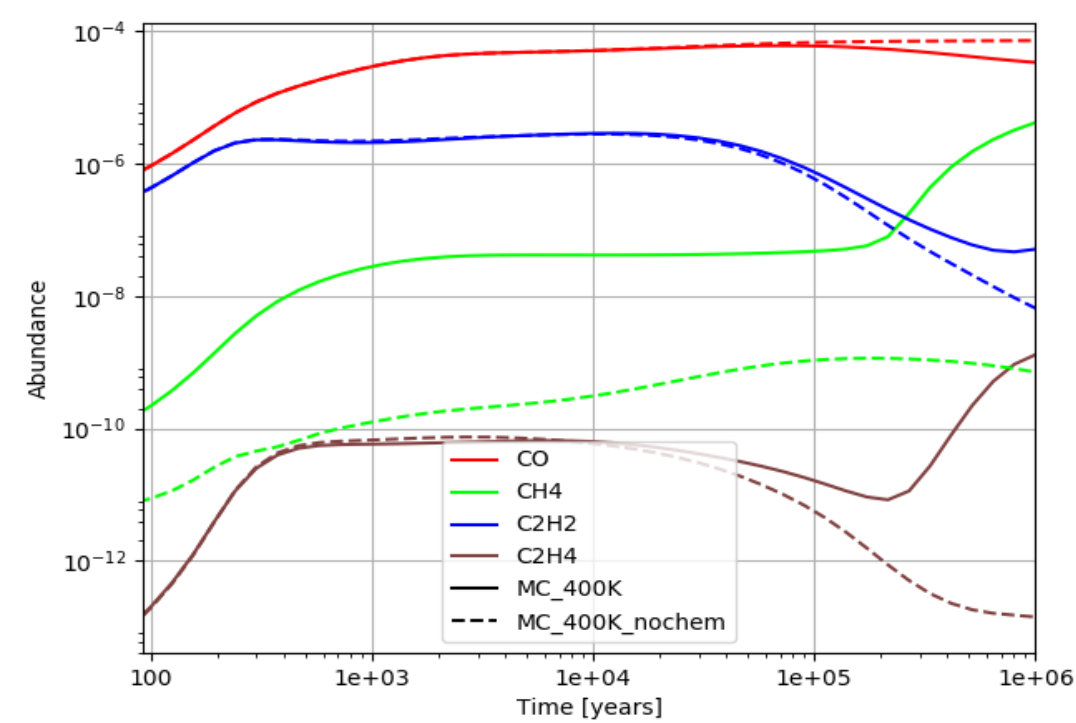

Figure 3.9: Fractional abundance of $\mathrm{CO}, \mathrm{CH}_{4}, \mathrm{C}_{2} \mathrm{H}_{2}$ and $\mathrm{C}_{2} \mathrm{H}_{4}$ with respect to $\mathrm{n}_{\mathrm{H}}$ as a function of time for a molecular cloud ran under standard conditions at $400 \mathrm{~K}$. 


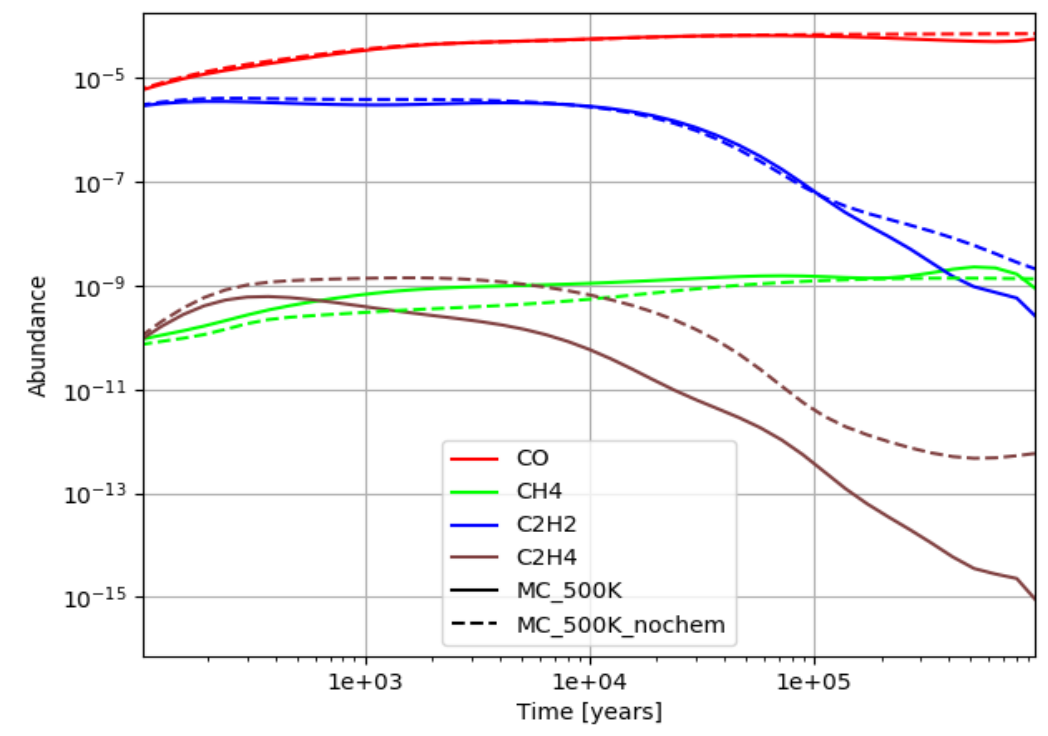

Figure 3.10: Fractional abundance of $\mathrm{CO}, \mathrm{CH}_{4}, \mathrm{C}_{2} \mathrm{H}_{2}$ and $\mathrm{C}_{2} \mathrm{H}_{4}$ with respect to $\mathrm{n}_{\mathrm{H}}$ as a function of time for a molecular cloud ran under standard conditions at $500 \mathrm{~K}$. 


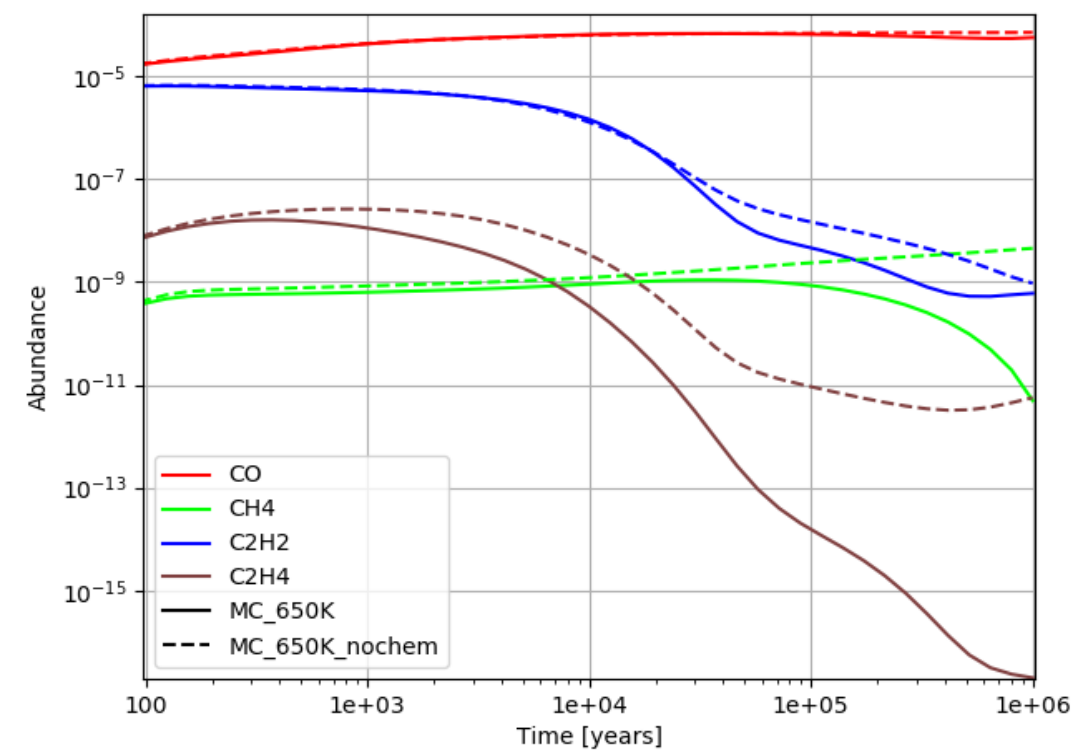

Figure 3.11: Fractional abundance of $\mathrm{CO}, \mathrm{CH}_{4}, \mathrm{C}_{2} \mathrm{H}_{2}$ and $\mathrm{C}_{2} \mathrm{H}_{4}$ with respect to $\mathrm{n}_{\mathrm{H}}$ as a function of time for a molecular cloud ran under standard conditions at $650 \mathrm{~K}$. 


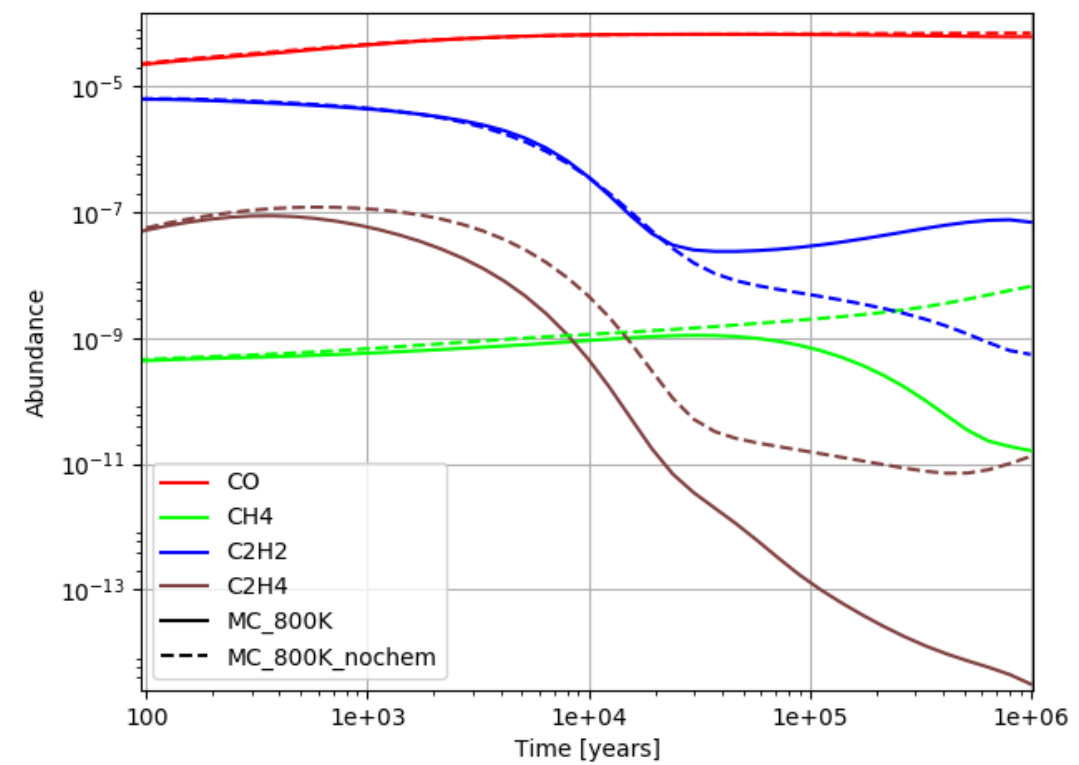

Figure 3.12: Fractional abundance of $\mathrm{CO}, \mathrm{CH}_{4}, \mathrm{C}_{2} \mathrm{H}_{2}$ and $\mathrm{C}_{2} \mathrm{H}_{4}$ with respect to $\mathrm{n}_{\mathrm{H}}$ as a function of time for a molecular cloud ran under standard conditions at $800 \mathrm{~K}$. 


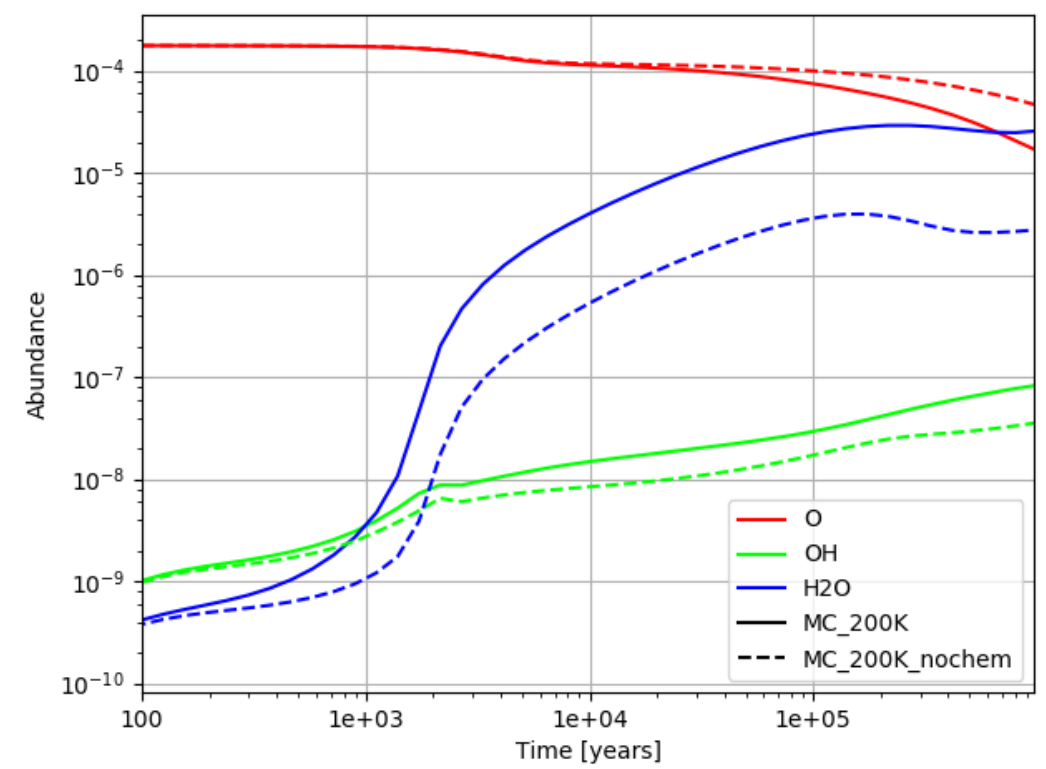

Figure 3.13: Fractional abundance of $\mathrm{O}, \mathbf{O H}$, and $\mathrm{H}_{2} \mathrm{O}$ with respect to $\mathrm{n}_{\mathrm{H}}$ as a function of time for a molecular cloud ran under standard conditions at $200 \mathrm{~K}$. 


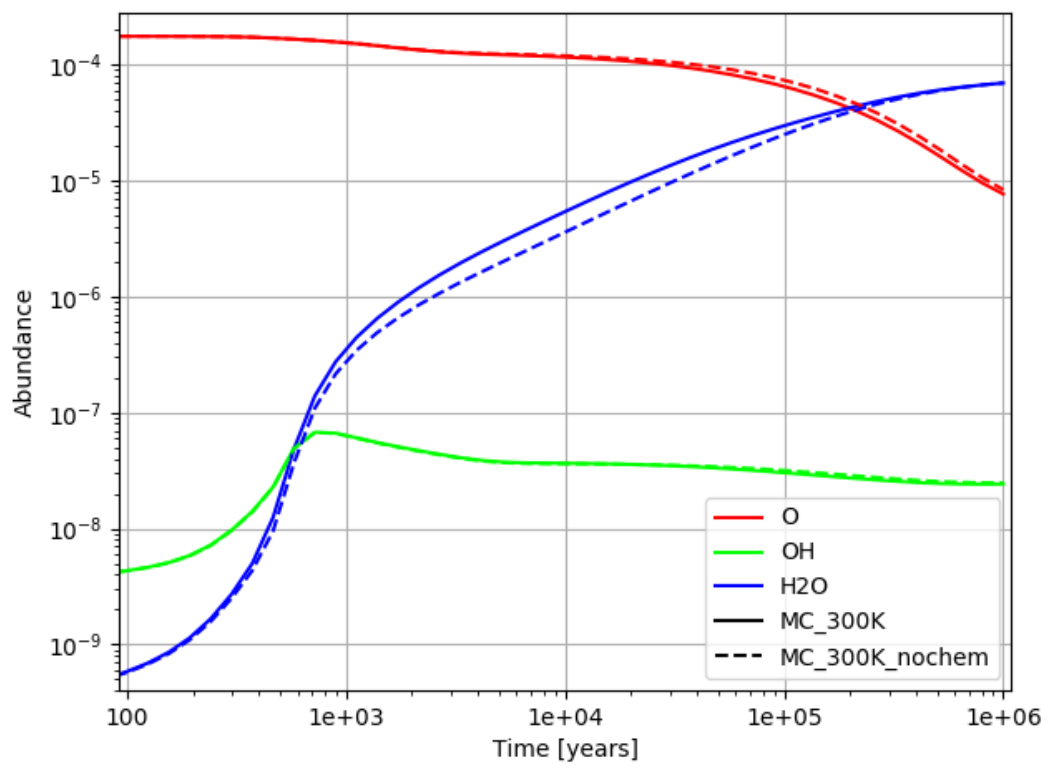

Figure 3.14: Fractional abundance of $\mathrm{O}, \mathrm{OH}$, and $\mathrm{H}_{2} \mathrm{O}$ with respect to $\mathrm{n}_{\mathrm{H}}$ as a function of time for a molecular cloud ran under standard conditions at $300 \mathrm{~K}$. 


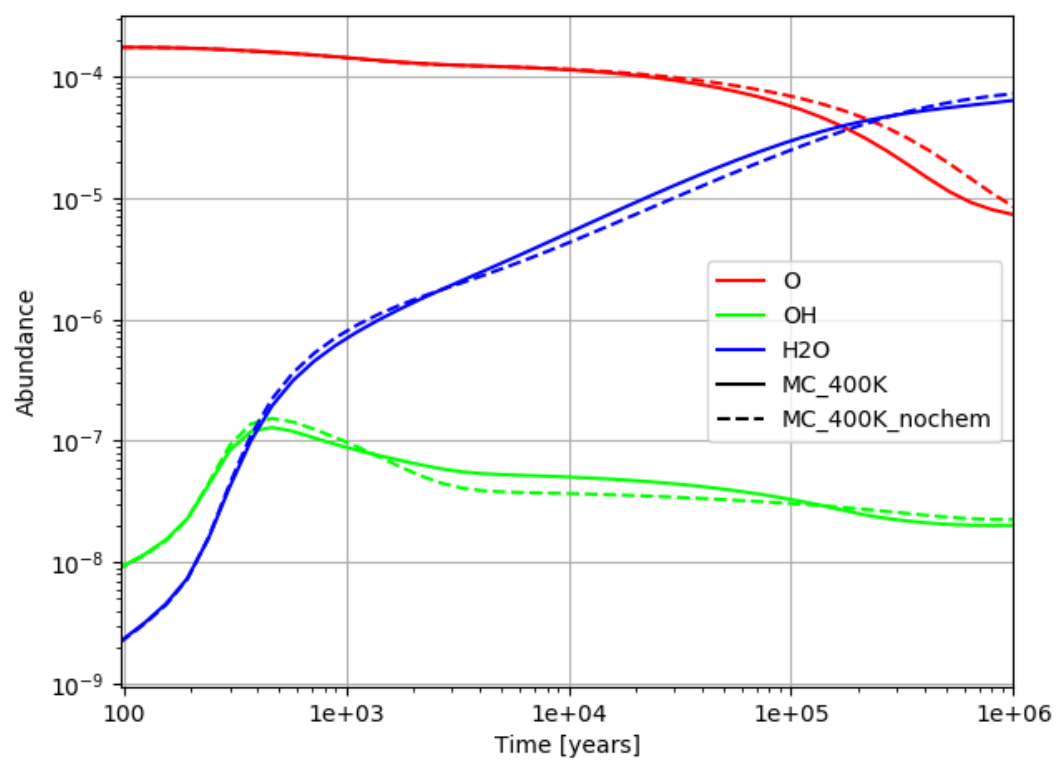

Figure 3.15: Fractional abundance of $\mathrm{O}, \mathrm{OH}$, and $\mathrm{H}_{2} \mathrm{O}$ with respect to $\mathrm{n}_{\mathrm{H}}$ as a function of time for a molecular cloud ran under standard conditions at $400 \mathrm{~K}$. 


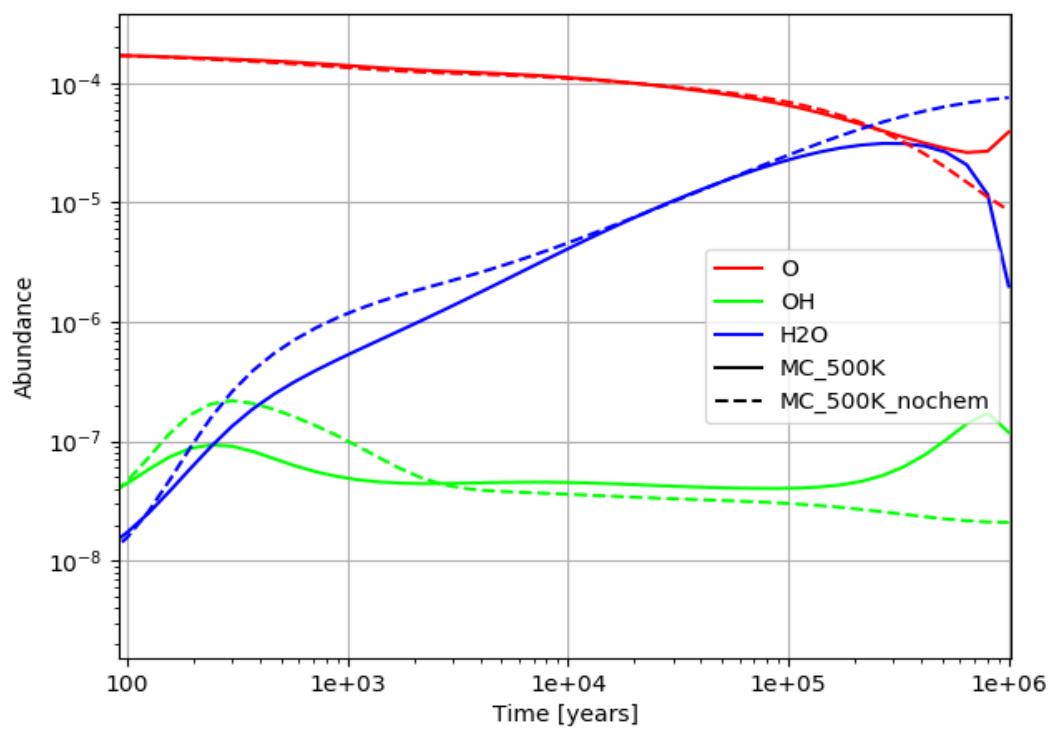

Figure 3.16: Fractional abundance of $\mathrm{O}, \mathrm{OH}$, and $\mathrm{H}_{2} \mathrm{O}$ with respect to $\mathrm{n}_{\mathrm{H}}$ as a function of time for a molecular cloud ran under standard conditions at $500 \mathrm{~K}$. 


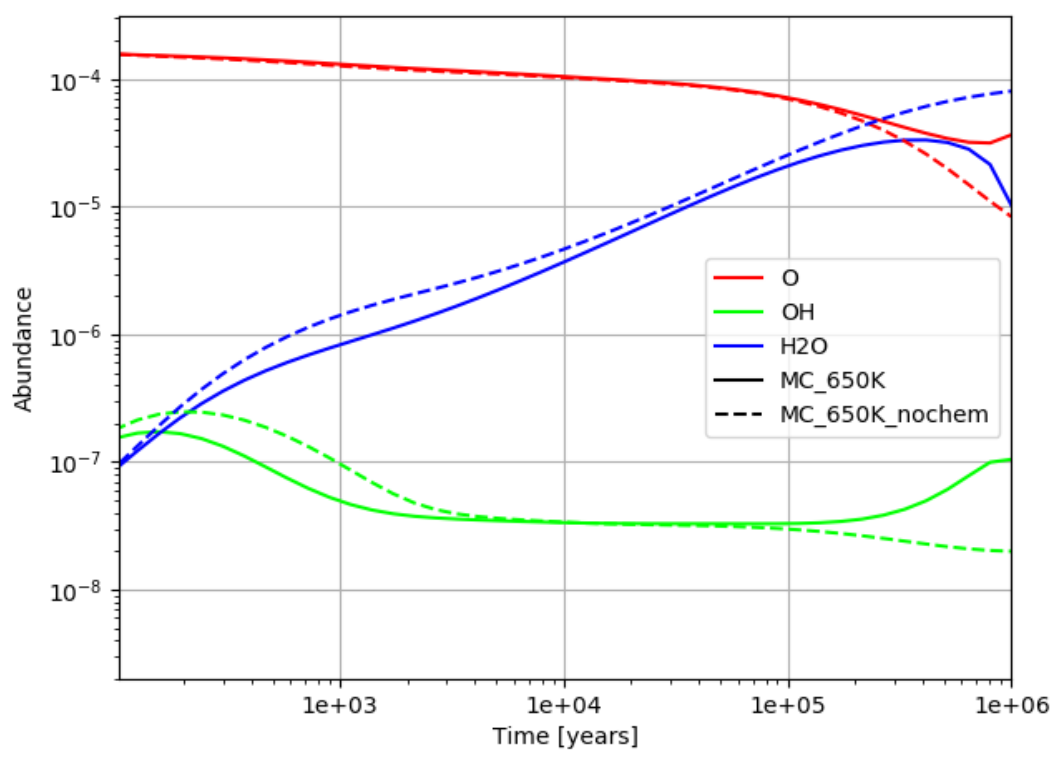

Figure 3.17: Fractional abundance of $\mathrm{O}, \mathrm{OH}$, and $\mathrm{H}_{2} \mathrm{O}$ with respect to $\mathrm{n}_{\mathrm{H}}$ as a function of time for a molecular cloud ran under standard conditions at $650 \mathrm{~K}$. 


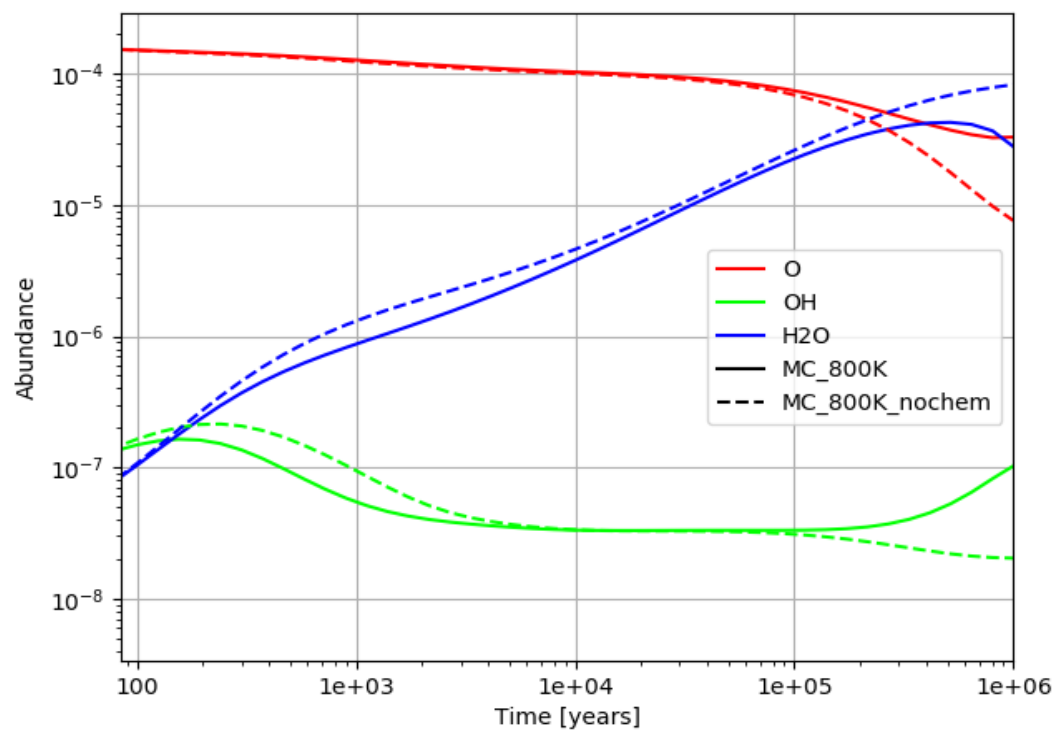

Figure 3.18: Fractional abundance of $\mathrm{O}, \mathrm{OH}$, and $\mathrm{H}_{2} \mathrm{O}$ with respect to $\mathrm{n}_{\mathrm{H}}$ as a function of time for a molecular cloud ran under standard conditions at $800 \mathrm{~K}$.

\begin{tabular}{|c|c|}
\hline $\begin{array}{c}\text { Table 3.2: Coverage on Grain Surface } \\
\text { at 10 }\end{array}$ \\
\hline & $\begin{array}{c}\text { Chears: } \\
\text { (monolayers (ML)) }\end{array}$ \\
\hline $\mathrm{T}(\mathrm{K})$ & 0.862313 \\
\hline 200 & 0.978403 \\
\hline 300 & 0.000415 \\
\hline 400 & 0.000013 \\
\hline 500 & 0.000002 \\
\hline 650 & 0.000000 \\
\hline 800 & \\
\hline
\end{tabular}


In figures 3.1-3.18 the dashed line represents fractional abundances as a function of time when the model was run without considering the new chemisorption processes (ie 'nochem' in figures). The solid line represent fractional abundances as a function of time when both chemisorption and physisorption processes are considered. In the above figures MC stands for molecular cloud. Throughout this paper ' $B$ ' represents species chemisorbed to the grain surface and ' $\mathrm{J}$ ' represents species physisorbed to the grain surface. The exceptions are $\mathrm{BH}_{2}, \mathrm{BC}_{2}, \mathrm{BO}_{2}$ and $\mathrm{BH}_{2} \mathrm{O}$ which are bound to the grain surface in a physisorption site. In the following few pages we will outline the mechanism for forming several species. In this we won't include some processes such as dissociation or reverse reaction but they have been included in the network and can be found in the appendix in Table A1. The dissociation process will be discussed in more detail in the discussion section.

When chemisorption isn't considered grain surface reactions become very inefficient in these high temperature regimes. Gas phase reactions become the main formation route for most species, especially complex organic molecules. ${ }^{34}$ This is because the binding energies are very low for most species $(\sim .1 \mathrm{eV})$ which leaves the grain surface relatively bare and most physisorption processes innefficient. However, even gas phase reactions in these high temperature regimes can be unfavorable. Considering these chemisorption processes may be important in producing some species in astrophysical environments. 
Molecular hydrogen is the most abundant molecule in the universe and dominates a lot of the chemistry in astrophysical environments. It's also a precursor in the formation of many complex organic molecules. It's therefore necessary to explore the production and destruction when considering chemisorption. Molecular hydrogen is formed most efficiently on the grain surface and through the following mechanism:

(3.1) $\mathrm{H} \rightarrow * \mathrm{H}$

(3.2) $2 * \mathrm{H} \rightarrow * \mathrm{H}_{2}\left(\mathrm{E}_{\mathrm{A}}=0.0 \mathrm{~K}\right)$

(3.3) $2 * \mathrm{H} \rightarrow \mathrm{H}_{2}$

(3.4) $* \mathrm{H}_{2} \rightarrow \mathrm{H}_{2}$

(3.5) $\mathrm{H}_{2} \rightarrow \mathrm{BH}+\mathrm{BH}$

where * is for species accreted onto the grain surface, either weakly bound $(\mathrm{J})$ or through a chemical bond (B). Dissociative adsorption (equation 3.5) only occurs when the incoming species has sufficient thermal energy to dissociate spontaneously upon contact with the grain surface.From 200-300 K (Figures 3.1-3.2) the chemisorption and physisorption processes are competitive with each other, with rates that have the same order of magnitude. The overall abundance of molecular hydrogen increases slightly in this regime because of the additional formation route for high temperature gas-grain chemistry. From $400-800 \mathrm{~K}$ by considering these additional chemisorption processes the amount of molecular hydrogen available in the gas phase decreases by up to almost two orders of magnitude from 500-800 K. From 200-400 K molecular 
hydrogen is destroyed in the same manner, dissociative adsorption or molecular adsorption. At $400 \mathrm{~K}$ the rate of destruction for dissociative adsorption and molecular adsorption increases by 2 orders of magnitude and 4 orders of magnitude, respectively, therefore decreasing the amount of molecular hydrogen available in the gas phase. From $500-800 \mathrm{~K}$ dissociative adsorption of molecular hydrogen is the dominant destruction mechanism.. From $400-800 \mathrm{~K}$ the dominant formation route for molecular hydrogen is thermal desorption of molecular hydrogen. Molecular hydrogen is weakly bound to the grain surface with a binding energy of $440 \mathrm{~K}$. The activation energy for reaction 3.2 is taken from work by Jelea et al. for the chemisorption case, for physisorption the reaction is also assumed to be barrierless. ${ }^{53}$ Molecular hydrogen has been known to be over produced in the gas phase using the rate equation approach. ${ }^{25,49-51}$ Iqbal et al. explored the formation of molecular hydrogen using the monte carlo approach, where they considered both chemisorption and physisorption sites. Considering these new chemisorption processes in this model only decreased the rate of formation roughly an order of magnitude, which is still much higher then what has been found using the Monte Carlo approach by lqbal et al. ${ }^{25}$ However, by considering chemisorption processes the abundance of molecular hydrogen decreases by almost two orders of magnitude due to these additional destruction pathways. This should have a significant impact on the gas phase chemistry since water has been known to form through hydrogenation reactions, as follows: ${ }^{54}$

(3.6) $\mathrm{O}+\mathrm{H}_{2} \rightarrow \mathrm{OH}+\mathrm{H}(\gamma=3160 \mathrm{~K})$

(3.7) $\mathrm{OH}+\mathrm{H}_{2} \rightarrow \mathrm{H}_{2} \mathrm{O}+\mathrm{H}(\gamma=1040 \mathrm{~K})$ 
When chemisorption processes aren't considered the abundance of both molecular and atomic hydrogen hits a steady state. Most the hydrogen is in the form of molecular hydrogen. By considering chemisorption processes this changes and the abundance of atomic hydrogen increases up to four orders of magnitude. The change in fractional abundance of atomic hydrogen isn't seen until $400 \mathrm{~K}$ since it binds to the grain surface with a binding energy of $14,000 \mathrm{~K}$ and remains on the grain surface until higher temperatures at which point thermal desorption becomes efficient.

Harada et al. found by only considering high temperature gas phase chemistry molecular hydrogen isn't destroyed in these high temperature regimes. ${ }^{34}$ As a result they found the production of water to be unaffected as well. This can have a huge affect on the chemistry in these environments because if oxygen is locked up in the form of water that means less atomic oxygen will be available in the gas phase. The amount of atomic oxygen in the gas phase plays an important role in the destruction of alkanes.

Atomic oxygen is also a key precursor in the production of $\mathrm{CO}$ at high temperatures. For example consider the following neutral-neutral reaction, which is the primary formation route for $\mathrm{CO}$ at $800 \mathrm{~K}$ in this model:

(3.8) $\mathrm{O}+\mathrm{CH}_{2} \rightarrow \mathrm{CO}+\mathrm{H}+\mathrm{H}$

Both water $\left(\mathrm{H}_{2} \mathrm{O}\right)$ and the hydroxyl radical $(\mathrm{OH})$ are formed most efficiently in the gas phase at these temperatures and the difference seen in their fractional abundances as a function of time is due to the change in atomic oxygen (see Figures 
3.13-3.18) from 500-800 K. Water weakly binds to the grain surface in a physisorption site with a binding energy of $5700 \mathrm{~K}$. According to Jelea et al. OH binds to the surface in a chemisorption site with a slightly higher binding energy of $6074 \mathrm{~K} .{ }^{53} \mathrm{In}$ both cases there isn't an appreciable amount of either species on the surface to make the grain-surface reaction efficient enough to compete with the gas phase reaction pathways at these temperatures. From $200-400 \mathrm{~K}$ the change in abundance of $\mathrm{OH}$ is due to the following reactive desorption reaction showing up as a secondary formation route:

(3.9) $\mathrm{BH}+\mathrm{BO} \rightarrow \mathrm{OH}$

Both atomic hydrogen and atomic oxygen bind to the grain surface in a chemisorption site so there's an appreciable amount of each species on the grain surface to make this reaction somewhat efficient before they begin to thermally desorb into the gas phase. Atomic oxygen (BO) binds to the surface of a grain with a binding energy of 29,046 K. Having this high of a binding energy causes some oxygen to be accreted onto the grain (see Figures A1-A6 in appendix for fractional abundances of species on the grain surface). From 200-400 K when considering these chemisorption processes you therefore see atomic oxygen become depleted from the gas phase. By $500 \mathrm{~K}$ oxygen begins to thermally desorb and from $500-800 \mathrm{~K}$ the abundance of oxygen is enhanced in the gas phase. When Harada et al. looked at high temperature gas phase chemistry without considering these gas-grain processes they found oxygen to be depleted from the gas phase in the form of water. ${ }^{34}$ 
Oxygen is also essential in the production of $\mathrm{OH}$ through high temperature gas phase chemistry. For example the following reaction is the main formation route for $\mathrm{OH}$ at $800 \mathrm{~K}$ :

(3.10) $\mathrm{O}+\mathrm{H}_{2} \rightarrow \mathrm{H}+\mathrm{OH}$

By increasing the reservoir of oxygen available in the gas phase you also see an increase in $\mathrm{OH}$. The decrease in abundance of water seen at high temperatures is due to an increase in destruction pathways, for example an increase in $\mathrm{H}$ and $\mathrm{C}^{+}$at $800 \mathrm{~K}$ will increase the rates of the following reactions:

(3.11) $\mathrm{H}+\mathrm{CH}^{+} \rightarrow \mathrm{H}_{2}+\mathrm{C}^{+}$

(3.12) $\mathrm{H}_{2} \mathrm{O}+\mathrm{C}^{+} \rightarrow \mathrm{H}+\mathrm{HCO}^{+}$

Carbon monoxide $(\mathrm{CO})$ is one of the most abundant molecules in astrophysical environments, second only in abundance to molecular hydrogen. ${ }^{36}$ It is the main gas phase reservoir of interstellar carbon and therefore controls much of the gas phase and grain surface chemistry in ISM, especially in the Fischer-Tropsch process which is a focus here. ${ }^{55}$ For this reason we thought it pertitent to explore it's fractional abundance when considering the new chemisorption processes added. From Figures 3.7-3.12 there doesn't appear to be a significant difference in gas phase abundances when considering this new process. This was unexpected since $\mathrm{CO}$ is a precursor to more complex molecules. The amount of carbon locked in the form of $\mathrm{CO}$ as opposed to $\mathrm{C}$ or $\mathrm{C}^{+}$will determine the abundance of small and large carbon-chain molecules. ${ }^{36,56}$ The 
slight decrease in gas phase abundance is simply due to CO accreting into a chemisorption site on the grain surface with a binding energy of 40,258 K. CO then dissociates on the grain into atomic carbon and oxygen which are bound in chemisorption sites on the grain surface. Atomic carbon begins to be enhanced in the gas phase by more then an order of magnitude past $400 \mathrm{~K}$ when comparing these results to models ran without considering chemisorption. Ionic carbon is also enhanced in the gas phase past $500 \mathrm{~K}$ by almost an order of magnitude through ion-neutral reactions due to the enhancement of atomic hydrogen in the gas phase. Carbon in both it's atomic and ionic form can act as a precursor in breaking down alkanes in these regimes.

Harada et al. found high temperature gas phase chemistry sufficient to reproduce molecular abundances around an active galactic nuclei (AGN) in some cases, but insufficient to explain CN/CO and other column density ratios. ${ }^{34}$ The new chemisorption formation rates for CO don't compete with the high temperature gas phase reactions at any of the given temperatures. However, we've only added 18 new chemisorbed species and 316 reactions, and the largest carbon chain molecule currently chemisorbed to a grain surface is ethene. The issue may just be more reactions and species need to be added, specifically complex organic molecules before this process can be thoroughly evaluated and compared with the results of Harada et al. 
In a 2001 paper Kress et al. looked at the Fischer-Tropsch catalysis in some detail under astrophysical conditions. ${ }^{3}$

Fischer-Tropsch catalysis is the conversion of $\mathrm{CO}$ and $\mathrm{H}_{2}$ into alkanes using a transition metal catalyst, which in our case is the dust grain. Kress et al. ran a rather simplistic model of 19 reactions and didn't consider gas phase chemistry in this regime. We've taken another look at this process for alkane formation in these high temperature regimes when both gas phase and grain surface chemistry is considered. Figures 3.7-3.12 show the fractional abundance of methane $\left(\mathrm{CH}_{4}\right)$, ethene $\left(\mathrm{C}_{2} \mathrm{H}_{4}\right)$ and acetylene $\left(\mathrm{C}_{2} \mathrm{H}_{2}\right)$ as a function of time at various temperatures.

The gas phase abundance of $\mathrm{CH}_{4}$ is significantly effected when considering chemisorption. The following is the mechanism of formation for $\mathrm{CH}_{4}$ on the grain surface when considering chemisorption:

$$
\begin{aligned}
& (3.13) \mathrm{C} \rightarrow \mathrm{BC} \\
& (3.14) \mathrm{H} \rightarrow \mathrm{BH} \\
& (3.15) \mathrm{BC}+\mathrm{BH} \rightarrow \mathrm{BCH}\left(\mathrm{E}_{\mathrm{A}}=0.0 \mathrm{~K}\right) \\
& (3.16) \mathrm{BC}+\mathrm{BH} \rightarrow \mathrm{CH} \\
& (3.17) \mathrm{BCH}+\mathrm{BH} \rightarrow \mathrm{BCH}_{2}\left(\mathrm{E}_{\mathrm{A}}=7.70 \mathrm{e}+03 \mathrm{~K}(\mathrm{EPR})\right) \\
& (3.18) \mathrm{BCH}+\mathrm{BH} \rightarrow \mathrm{CH}_{2} \\
& (3.19) \mathrm{BCH}_{2}+\mathrm{BH} \rightarrow \mathrm{BCH}_{3}\left(\mathrm{E}_{\mathrm{A}}=5.54 \mathrm{e}+03 \mathrm{~K}\right) \\
& (3.20) \mathrm{BCH}_{2}+\mathrm{BH} \rightarrow \mathrm{CH}_{3}
\end{aligned}
$$


(3.21) $\mathrm{BCH}_{3}+\mathrm{BH} \rightarrow \mathrm{BCH}_{4}\left(\mathrm{E}_{\mathrm{A}}=0.0 \mathrm{~K}\right)$

(3.22) $\mathrm{BCH}_{3}+\mathrm{BH} \rightarrow \mathrm{CH}_{4}$

(3.23) $\mathrm{BCH}_{4} \rightarrow \mathrm{CH}_{4}$

We've left out thermal desorption processes and other less efficient formation routes but these can be found in table A1. Due to the mobility of hydrogen this is the most efficient route on the grain surface when compared with larger radical species that are less mobile. The barrier for reaction 3.19 was taken from a paper by Rodriguez et al. ${ }^{57}$ Reactions 3.15 and 3.21 were treated in accordance with the work of Calderon et al. ${ }^{58}$ For reaction 3.17 a literature value wasn't available so the barrier was estimated using the Evan's-Polanyi Relationship. ${ }^{17}$ The drawbacks to this approach will be discussed later in this thesis.

At $200 \mathrm{~K}$ the gas phase abundance of $\mathrm{CH}_{4}$ increases up to 4 orders of magnitude at late times ( $10^{6}$ years) and 1 order of magnitude at early times (100 years). Reaction 3.24, thermal desorption of a weakly bound methane species, produces roughly half of the methane being produced at later times $\left(10^{5}-10^{6}\right.$ years, Figure 3.7):

(3.24) $\mathrm{JCH}_{4} \rightarrow \mathrm{CH}_{4}$

Initially this seems counterintuitive since the binding energy of physisorbed methane is $1300 \mathrm{~K}$. The efficiency of this process is due to the fact that the thermal velocity of species ' $i$ ' in the gas phase is proportional to the square root of the gas phase temperature (equation 2.18), making accretion an efficient process in this regime. Due to this increase in flux there's 
.000223 monolayer (ML) coverage of physiosorbed species at this temperature regime at later times. Reaction 3.25 below is a dissociative recombination reaction (section 2.1.4), and is only important in early times (1-100 years) and is a secondary formation route in this time frame:

(3.25) $\mathrm{C}_{2} \mathrm{H}_{4}^{+}+\mathrm{e}^{-} \rightarrow \mathrm{C}+\mathrm{CH}_{4}$

Dissociative recombination reactions are typically fast when compared with radiative recombination and is actually the main formation route for methane at early times when chemisorption isn't considered. The main formation route for methane at $200 \mathrm{~K}$ is reactive desorption, reaction 3.22, which is described in more detail in section 2.2 .5 above. As stated previously, to calculate the fraction of reactions resulting in desorption we assumed a value of 1.0 for the value ' $a$ ' in equation 2.29. Equation 2.29 considers the competition between the rate of desorption and the rate of energy lost to the grain surface. Equation 2.30 gives the probability of desorption to occur and is a function of the binding energies of the reactants and enthalpy of formation of the reactants. Enthalpy of formation is estimated using Benziger's approach. ${ }^{17}$ Binding energies for atomic hydrogen $(\mathrm{BH})$ and the methyl radical $(\mathrm{BCH} 3)$ were taken from literature and were $14,000 \mathrm{~K}$ and $10,259 \mathrm{~K}$, respectively. 51,58

From 300-500 $\mathrm{K}$ thermal desorption of $\mathrm{CH}_{4}$ is the main formation route (equation 3.23 above). The binding energy was taken from a paper by Liu et al $(22,070 \mathrm{~K}) .{ }^{59}$ Liu et al. studied the activation of methane on a graphite surface and looked 
at 16 different initial adsorption configurations. The value we use is from the most stable configuration. However, clearly this approach has its drawbacks, we're assuming every site on the grain where the adsorbate binds is the most stable possible configuration. In general, most species tend to be activated on defective graphite surfaces and using the rate equation approach this isn't taken into consideration in this model. This will be discussed in more detail in the discussion section. At $300 \mathrm{~K}$ the grain surface is relatively saturated with .978 monolayers (ML) of coverage (Table 3.2). At $400 \mathrm{~K}$ there's still $.000415 \mathrm{ML}$ coverage. In the appendix we've included model results of carbon species on the grain surface at various temperatures (Figures A1-A6). At $400 \mathrm{~K}$ the rate of thermal desorption dominates dissociative recombination reactions by about 3 orders of magnitude. Past $400 \mathrm{~K}$ the grain becomes relatively bare and dissociative recombination reaction become the main formation route for $\mathrm{CH}_{4}$ production. By including the new chemisorption processes the overall abundance of methane increases from $200-500 \mathrm{~K}$ and decreases at both $650 \mathrm{~K}$ and $800 \mathrm{~K}$. The decrease in abundance is due to these environments becoming essentially oxygen rich which causes an efficient breakdown of large carbon chain molecules. ${ }^{34}$ At both $650 \mathrm{~K}$ and $800 \mathrm{~K}$ The following neutral-neutral reaction becomes more efficient due to the increase in atomic oxygen in the gas phase:

(3.26) $\mathrm{O}+\mathrm{CH}_{4} \rightarrow \mathrm{OH}+\mathrm{CH}_{3}$ 
When considering the new high temperature gas-grain chemistry the fractional abundance of $\mathrm{C}_{2} \mathrm{H}_{2}$ increases from 200-400 K and at $800 \mathrm{~K}$ and decreases at $500 \mathrm{~K}$ and $650 \mathrm{~K}$ (Figures 3.7-3.12). In each situation this happens at late times $\left(10^{5}-10^{6}\right.$ years). The gas-grain formation and destruction routes aren't competitive with the high temperature gas phase reactions so the difference in abundances is due to an enhancement of atomic oxygen and molecular hydrogen in the gas phase which is most prevalent at late times (Figures 3.13-3.18 and 3.1-3.6). This is the most effective way to destroy long chain carbons and is a result of atomic oxygen being enhanced in the gas phase from 500-800 K. Atomic oxygen being enhanced in the gas phase can also affect formation routes. At $800 \mathrm{~K}$ the following reaction is the main formation route for $\mathrm{C}_{2} \mathrm{H}_{2}$ :

(3.27) $\mathrm{O}+\mathrm{CH}_{2} \mathrm{CCH} \rightarrow \mathrm{HCO}+\mathrm{C}_{2} \mathrm{H}_{2}$

There's also an enhancement of molecular hydrogen that increases the rate of the following reaction from 200-400 K:

(3.28) $\mathrm{H}_{2}+\mathrm{CCH} \rightarrow \mathrm{H}+\mathrm{C}_{2} \mathrm{H}_{2}$

The fractional abundance of $\mathrm{C}_{2} \mathrm{H}_{4}$ also changes drastically by considering these new processes. The fractional abundance of $\mathrm{C}_{2} \mathrm{H}_{4}$ increases from 200-400 K and decreases from 500-800 K (Figures 3.7-3.12). The grain-surface formation route also isn't competitive with gas phase reactions here either. The difference in abundance is once aga in due to the 
difference in gas phase oxygen which increases the rate of destruction from 500-800 K. From 200-400 K the enhancement of $\mathrm{C}_{2} \mathrm{H}_{4}$ is due to additional $\mathrm{CH}_{4}$ in the gas phase and the following reaction becoming more efficient:

(3.29) $\mathrm{CH}+\mathrm{CH}_{4} \rightarrow \mathrm{H}+\mathrm{C}_{2} \mathrm{H}_{4}$

For $\mathrm{C}_{2} \mathrm{H}_{4}$ having enhanced amounts of atomic carbon and ionic carbon in the gas phase increases destruction processes. This was touched on previously when looking at the abundance of $\mathrm{CO}$ in the gas phase. For example, the following reactions become efficient at $800 \mathrm{~K}$ :

$$
\begin{aligned}
& \text { (3.30) } \mathrm{C}^{+}+\mathrm{C}_{2} \mathrm{H}_{4} \rightarrow \mathrm{C}+\mathrm{C}_{2} \mathrm{H}_{4}^{+} \\
& \text {(3.31) } \mathrm{C}+\mathrm{C}_{2} \mathrm{H}_{4} \rightarrow \mathrm{H}+\mathrm{CH}_{2} \mathrm{CCH}
\end{aligned}
$$

Harada et al. found that when including high temperature gas phase chemistry the system becomes essentially "carbon rich" because of the lack of atomic oxygen in the gas phase, which leads to long chain carbons not being destroyed as efficiently. ${ }^{34}$ By including chemisorption processes we found an increase in atomic oxygen in the gas phase at high temperatures and a depletion of water, under the same conditions they ran their model under. 


\subsection{Eley-Rideal (ER) Processes:}

In an Eley-Rideal (ER) reaction mechanism species ' $A$ ' accretes onto a grain surface and then reacts with an incoming ' $B$ ' species from the gas phase (see Figure 1.2 and section 1.3). We ran models using the same physical conditions described previously in section 3.1. We checked the fractional abundance of $\mathrm{H}, \mathrm{H}_{2}, \mathrm{O}, \mathrm{OH}, \mathrm{H}_{2} \mathrm{O}, \mathrm{CO}, \mathrm{CH}_{4}, \mathrm{C}_{2} \mathrm{H}_{2}$ and $\mathrm{C}_{2} \mathrm{H}_{4}$ as a function of time at $200,300,400,500,650$ and $800 \mathrm{~K}$. For the first model we used a diffusion to desorption barrier of .2. When this low barrier was used the Eley-Rideal (ER) process didn't compete with the Langmuir Hinchelwood (LH) reaction mechanism for any species considered. We ran a second model and increased the diffusion to desorption barrier to .99, we were attempting to render the adsorbate ' $A$ ' relatively static on the grain surface. This should have decreased the efficiency of the $\mathrm{LH}$ reaction mechanism by rendering the diffusion process rather inefficient. By decreasing the LH reaction rate, the ER reaction rate should start to be a competitive mechanism. Although the rate of the LH reaction mechanism did decrease the rate of the ER mechanism didn't compete with it under these circumstances. There's two main driving forces in the efficiency of an ER mechanism. At high coverage, the gas phase species has a higher probability of encountering an adsorbate upon contact with the grain surface. Another deciding factor in the efficiency of an ER process is the flux, which is modeled by a Maxwellian velocity distribution which has a temperature dependence $\left(\propto \mathrm{T}^{1 / 2}\right)$. Even at low coverage the ER process may be efficient because of an increase in collisions per second. Since this network is rather incomplete, 18 species and 316 reactions have been added, the inability of the ER mechanism to compete with the LH mechanism may just be because more chemisorption 
processes and species need to be added. Once that happens the grain won't be as bare and the probability of a gas phase species encountering an adsorbate on the grain will increase. It's also worth noting we use a hydrogen atom density of $10^{4} \mathrm{~cm}^{-3}$, using a higher density would increase the flux and in theory increase the ER reaction rate. In star forming regions like protoplanetary disk a higher density is likely $\left(\sim 10^{5} \mathrm{~cm}^{-3}\right)$, so this process may be important in astrophysically relevant environments. ${ }^{60}$

\subsection{Discussion:}

In the previous section we presented results from a modified version of the three-phase gas-grain model presented in detail by Ruaud et al. 6, 21 The third phase was turned off so mantle chemistry was not considered. The modifications made were necessary to explore high temperature gas-grain chemistry and explore how coupling this with gas phase processes will affect molecular abundances of molecules in astrophysical environments. We ran a model for a molecular cloud under standard conditions and compared the results to those of a model without considering chemisorption processes, in which case the main formation and destructing route would be gas phase chemistry. We mainly wanted to explore the fractional abundances of alkanes $\left(\mathrm{CH}_{4}, \mathrm{C}_{2} \mathrm{H}_{2}, \mathrm{C}_{2} \mathrm{H}_{4}\right)$, previous work by Kress et al. claimed the Fischer Tropsch process would be the most likely formation route for alkanes at high temperatures and the most efficient way to convert CO into other carbon compounds. ${ }^{3}$ We also explored the abundances of $\mathrm{H}_{2} \mathrm{H}_{2}, \mathrm{CO}, \mathrm{O}, \mathrm{OH}$ and $\mathrm{H}_{2} \mathrm{O}$. We were expecting a change in fractional 
abundances that, when augmented with both observational data and our knowledge of astrophysical environments, would offer insight into the importance of these new processes. We didn't get a chance to compare model results to observational abundances for a couple different reasons. One was there were to many uncertainties in the model to make any drastic conclusions, which will be elaborated on in this section. The second was an issue we were having with the ordinary differential equation (ODE) solver. Nautilus uses an ODE solver called the Double Precision Livermore Solver of Ordinary Differential Equations (DLSODES). By adding these new processes, the system of differential equations becomes stiff and wouldn't converge. DLSODES uses the GEAR method to solve ODE's, we tried implementing a different ODE solver with a similar interface but that didn't alleviate the issue. Specifically, we were having issues at temperatures below $200 \mathrm{~K}$ and at higher densities $\left(>10^{6} \mathrm{~cm}^{-3}\right)$. For this reason, modelling a protoplanetary disk or hot core with varying physical conditions would have proved problematic. ${ }^{5,61}$ Since these are the environments these new processes may prove to be important this is an issue that needs to be addressed.

Although we weren't able to compare model results to observational abundances to see how important these new chemisorption processes were, this model was successful in some respects and it's important to start there. The change in fractional abundance of both molecular hydrogen and atomic oxygen seem reliable. In summary, for molecular hydrogen the abundance increased slightly from 200-300 K due to additional formation routes added, and then decreased once dissociative adsorption began to be a dominate destruction route from $400-800 \mathrm{~K}$. Since atomic hydrogen is bound to the 
grain surface in a chemisorption site, the gas-grain formation route is quite efficient. The fact that molecular hydrogen binds to a graphite surface in a physisorption site, atomic hydrogen binds in a chemisorption site and molecular hydrogen dissociatively absorbs onto the surface is well established in literature and we therefore believe these processes can be trusted. ${ }^{39,53,57-58,62}$ It's worth noting we used a value of 14,000 $\mathrm{K}$ for the binding energy of atomic hydrogen in accordance with the work of Cazaux et al. ${ }^{51}$, this value is likely quite high, Petucci et al. ${ }^{63}$ found the entrance barrier for atomic hydrogen to chemisorb on graphite to be $.2 \mathrm{eV}$ and this value seems to be well established by multiple groups and should be adjusted accordingly. ${ }^{39,53,57-58,62}$ Considering these new chemisorption processes in this model only decreased the rate of formation roughly an order of magnitude, which is still much higher then what has been found using the Monte Carlo approach by lqbal et al. ${ }^{25}$ It's important to note lqbal et al. used a different hydrogen density, varied the grain size in their model and considered that species bind in a physisorbed precursor state before diffusing to a chemisorbed site. Since the models were run under different conditions comparing the results is problematic. This change in the abundance of molecular hydrogen had drastic consequences in the efficiency of some gas phase processes. Molecular hydrogen is a precursor in the formation of water which can be the dominant form of oxygen if molecular hydrogen is overproduced. Since this is not the case we thought it pertinent to look at atomic oxygen in the gas phase, since that is a key precursor in breaking down large chain alkanes. 
We found the abundance of atomic oxygen to decrease from $200-400 \mathrm{~K}$ and then increase from $500-800 \mathrm{~K}$. There are two contributing factors for this. One is the binding energy of atomic oxygen (BO), which according to Jelea et al. binds to the surface of a grain with a binding energy of $29,046 \mathrm{~K} .{ }^{53}$ Having this high of a binding energy causes some oxygen to be accreted onto the grain (see Figures A1-A6 in appendix for fractional abundances of species on the grain surface). As a result atomic oxygen will be depleted from the gas phase until it has enough energy to thermally desorb, which seem to take place at $500 \mathrm{~K}$. The second contributing factor to the change in atomic oxygen in the gas phase has to do with gas-grain formation routes for $\mathrm{OH}$ and $\mathrm{H}_{2} \mathrm{O}$. According to Jelea et al. $\mathrm{H}_{2} \mathrm{O}$ weakly binds to the grain surface in a physisorption site with a binding energy of $5700 \mathrm{~K}$ and $\mathrm{OH}$ binds to the surface in a chemisorption site with a slightly higher binding energy of $6074 \mathrm{~K} .{ }^{53} \mathrm{In}$ both cases there isn't an appreciable amount of either species on the surface to make the grain-surface reaction efficient enough to compete with the gas phase reaction pathways from 500-800 K. However, OH forms efficiently from reactive desorption from $200-400 \mathrm{~K}$ since both atomic hydrogen and oxygen bind to the grain surface in a chemisorption site. Atomic oxygen being bound to the grain surface in a chemisorption site is well established, as is molecular oxygen being bound in a physisorption site. Moron et al. found the adsorption energy of atomic oxygen to fall within the range of 1.5-3.2 eV depending on surface coverage, and our value falls within that window. ${ }^{64}$ Atomic oxygen is a necessary precursor in the destruction of large chain alkanes so this had a substantial impact on the results. We found the abundance of $\mathrm{CH}_{4}, \mathrm{C}_{2} \mathrm{H}_{2}, \mathrm{C}_{2} \mathrm{H}_{4}$ to be significantly depleted at higher temperatures because of this. 
The gas phase abundance of $\mathrm{CO}$ didn't change significantly by adding these new chemisorption processes. $\mathrm{CO}$ is a precursor to more complex molecules so any changes in abundance would affect the chemistry in these environment significantly. The amount of carbon locked in the form of $\mathrm{CO}$ as opposed to $\mathrm{C}$ or $\mathrm{C}^{+}$will determine the abundance of small and large carbon-chain molecules. ${ }^{36,56}$ There was a minor decrease in $\mathrm{CO}$ in the gas phase which can be attributed to it accreting onto the grain surface in a chemisorption site. There was also a slight increase in abundance of atomic carbon in the gas phase past $400 \mathrm{~K}$ which can be attributed to the chemisorbed species desorbing into the gas phase in this regime. Ionic carbon is also enhanced in the gas phase past $500 \mathrm{~K}$, this is due to the significant increase in atomic hydrogen which makes ion-neutral reactions that produce $\mathrm{C}^{+}$more efficient. Both of these species can impact the destruction of alkanes in the gas phase at these temperatures, but the difference in abundance wasn't significant.

Chemisorption is very site dependent, the defect site of a grain is typically the most reactive for instance. ${ }^{59}$ It's also worth noting that some closed shell species such as methane will chemisorb to the defect site of the grain surface but physisorb everywhere else. ${ }^{59}$ In this model this site specificity is overlooked for the time being. As a result the results for methane abundance are questionable. By considering the new chemisorption processes the abundance of methane in the gas phase increased substantially, especially from $200-400 \mathrm{~K}$, where the abundance increased roughly four orders of magnitude. However, methane only binds to a grain surface in a defect site and the rate equation approach has no way to take this into consideration. As a result we're likely over producing methane in this model since we're assuming it can bind anywhere on 
the grain surface. One solution to this problem would be using a continuous-time random-walk (CTRW) Monte Carlo technique similar to what was done by lqbal et al. ${ }^{25}$ Using this approach it's possible to follow the movement of the adsorbate and one can even move from physisorption to chemisorption sites.

There's also a lot of uncertainty when estimating barriers. The surface is also important when it comes to reactivity of certain reactions. ${ }^{59}$ Typically the defect site can be considered the most reactive but dissociation energies and activation energies can differ substantially just by surface and site dependences. ${ }^{18}$ The solution to this would be again abandoning the rate equation approach and using a CTRW Monte Carlo technique to track the adsorbate on the grain surface. For dissociation energies and activation energies we used some less then ideal techniques to estimate barriers. To estimate the dissociation energy we used what's known as the BOC-MP method. This approach isn't based on first principles and the theoretical justification isn't great. It can be quite accurate in certain instances, for instance it seems to work quite well in predicting scission of $\mathrm{O}-\mathrm{H}$ or $\mathrm{C}-\mathrm{H}$ but less well for $\mathrm{C}-\mathrm{C}$ or double or triple bonds. ${ }^{17}$ To estimate activation energies we used an Evans-Polanyi relation when a literature value wasn't available. ${ }^{17}$ Like the BOC-MP method this doesn't have a great theoretical justification. Both of these methods can be quite accurate but without verifying the barriers in a lab setting or doing a more rigorous quantum mechanical approach to verify the answer it won't necessarily hold up to scrutiny. Using these approaches the barrier can be off by as much as 1-2 eV, leading to significant error. ${ }^{17-18}$ For dissociation energies there was less literature available and we had to rely on the BOC-MP method almost exclusively. From 200-300 K photodissociation 
is the dominant dissociation process but beyond $300 \mathrm{~K}$ the rate is about $20-30$ orders of magnitude smaller then the thermal dissociation process. Since these barriers aren't well constrained this can't be trusted, meaning the rate at which alkanes a re both formed and destroyed on the grain surface is questionable. To attempt to address this shortcoming a more rigorous quantum approach could be taken. There is several ways to attempt to solve the Schrodinger equation and determine the electronic structure of atoms and molecules. One approach would be using ab-initio calculations where a model is chosen for the electronic wavefunction which is used to solve the electronic Schrodinger equation. ${ }^{65}$ This approach has been carried out by Woon et al. to study astrophysical ices and neutral interstellar molecules. ${ }^{32,66}$ Another approach, and one that is more commonly used in surface science, is density functional theory (DFT). ${ }^{17,53,58,65,67}$ The basic idea behind DFT is that the the energy of an electronic system can be written in terms of the elctron probability density, $\rho$, for a system of $\mathrm{N}$ electrons. There are several advantages to this approach. One is by using the electron density rather then a wavefunction approach one is able to circumvent some of the constraints normally imposed due to permutation symmetry. A single three dimensional function for an $\mathrm{N}$ electron molecule is used. Furthermore, an $\mathrm{N}$ electron wavefunction has cusp where the electrons approach one another whereas the electron density does not involve inter-electron distances explicitly and has no such cusp. The take home message is much more care needs to be taken in estimating barriers for these results to be considered reliable. 
The fractionable abundance of both $\mathrm{C}_{2} \mathrm{H}_{2}$ and $\mathrm{C}_{2} \mathrm{H}_{4}$ was due to enhancement of atomic oxygen in the gas phase as well as $\mathrm{H}_{2}, \mathrm{CH}_{4}, \mathrm{C}$ and $\mathrm{C}^{+}$. Atomic oxygen is needed for both formation and destruction of alkanes in the gas phase, but impacted the chemistry the most by providing efficient destruction routes. The new gas-grain processes added for $\mathrm{C}_{2} \mathrm{H}_{2}$ and $\mathrm{C}_{2} \mathrm{H}_{4}$ didn't compete with the gas phase reactions at any temperature considered. For these two alkanes in particular the rates are particularly questionable because there wasn't as much literature available to attempt to constrain the barriers. For both these molecules the binding energy was treated as a free paramter, the physisorption value was multiplied by 10 to give it the correct order of magnitude. However, it's not clear both of these molecules will bind to a grain surface in a chemisorption site. $\mathrm{C}_{2} \mathrm{H}_{4}$ for instance is a closed shell speccies and if it did bind to the grain surface in a chemisorption site this would likely occur at a defect site, similar to $\mathrm{CH}_{4} \cdot{ }^{59}$ For $\mathrm{C}_{2} \mathrm{H}_{2}$ Hosseinnejad et al. found it binds to a graphite basal surface in a physisorption site with a barrier of $.02 \mathrm{~kJ} / \mathrm{mol} .{ }^{68}$ However, they claim the same thing for $\mathrm{CO}$ and according to Strange et al. CO will bind in a chemisorption site. ${ }^{69}$ Liu et al. also claimed CO will bind in a chemisorption site on defective graphite, Strange et al. studied the CO reaction in 1976 over natural graphite and its possible there was defect sites on the graphite surface causing this strong interaction. This is again something that would be better suited for the CTRW monte carlo technique. 


\subsection{Future Directions:}

For physisorption processes adsorption is believed to be a barrierless process. We've made that same assumption for the chemisorption case. Recall the following equation from section 2.2.1:

$(2.14) \mathrm{k}_{\mathrm{ads}}(\mathrm{i})=\mathrm{S}(\theta) * \sigma_{\mathrm{d}} *\langle\mathrm{v}(\mathrm{i})\rangle * \mathrm{n}(\mathrm{i}) * \mathrm{n}_{\mathrm{d}}$

Accretion is normally treated by multiplying this equation by a Boltzmann factor that's a function of adsorption energy and grain temperature. ${ }^{17-18}$ Chemisorption processes are more likely to have a barrier for adsorption so this factor should be included for molecular adsorption. Assuming dissociative adsorption has no barrier is still valid.

Before observational results can be compared to model results this model's limitations need to be addressed. Specifically, ODE's become stiff and fail to converge at low temperatures and high densities. It may be necessary to use a different ODE solver to address this shortcoming. Constraining the barriers used in these reactions is also a necessity as touched on previously.

As I hope we've made clear in this thesis chemisorption is very surface and site dependent, defect sites are substantially more reactive and will even bind some closed shell species with a strong molecular bond.$^{59}$ The rate equation approach has no way to treat this so a CTRW monte carlo technique may be better suited to follow the path of the adsorbate explicitly. ${ }^{70}$ To stick with the rate equation approach one could assume closed shell species only physisorb on the grain 
surface, so essentially thermal desorption of methane at high temperatures would be negligible since it's binding energy is so low. However, it could still form from reactive desorption. Kress et al. studied the Fischer-Tropsch process at high temperatures and assumed it could only form from reactive desorption. ${ }^{3}$

There's an additional destruction route for molecular hydrogen that can be quite important and should be added to the network. In this process molecular hydrogen accretes onto a grain surface and dissociates, ejecting a hydrogen atom into the gas phase and leaving one bound in a chemisorption site. This bond cleavage is caused by a violently exothermic reaction. Calderon et al. found this process to have a barrier of $24.7 \mathrm{~kJ} / \mathrm{mol} .{ }^{58}$ Atomic hydrogen would need a free site to accrete into, so equation 2.17 would again be needed.

The results for this model are inconclusive in the mid temperature range (200-300 K) because of how diffusion from physisorption sites to chemisorption sites is being treated. For diffusion from a physisorption site to a physisorption site, or a chemisorption site to a chemisorption site, only horizontal movement needs to be considered. ${ }^{25,51}$ However when considering diffusion between chemisorption sites and physisorption sites a vertical component needs to be considered as well. This can be important when the adsorbate accretes into a precursor state before diffusing to a chemisorption site. The lifetime of a precursor state is only about $50 \mu$ s so the temperature range we gave for when this may be important is an estimate. In a 2004 paper Cazaux et al. use what's known as a transmission coefficient to handle diffusion both thermally and 
quantum mechanically in a rate equation model when considering the interaction between chemisorption and physisorption sites: ${ }^{12,} 51$

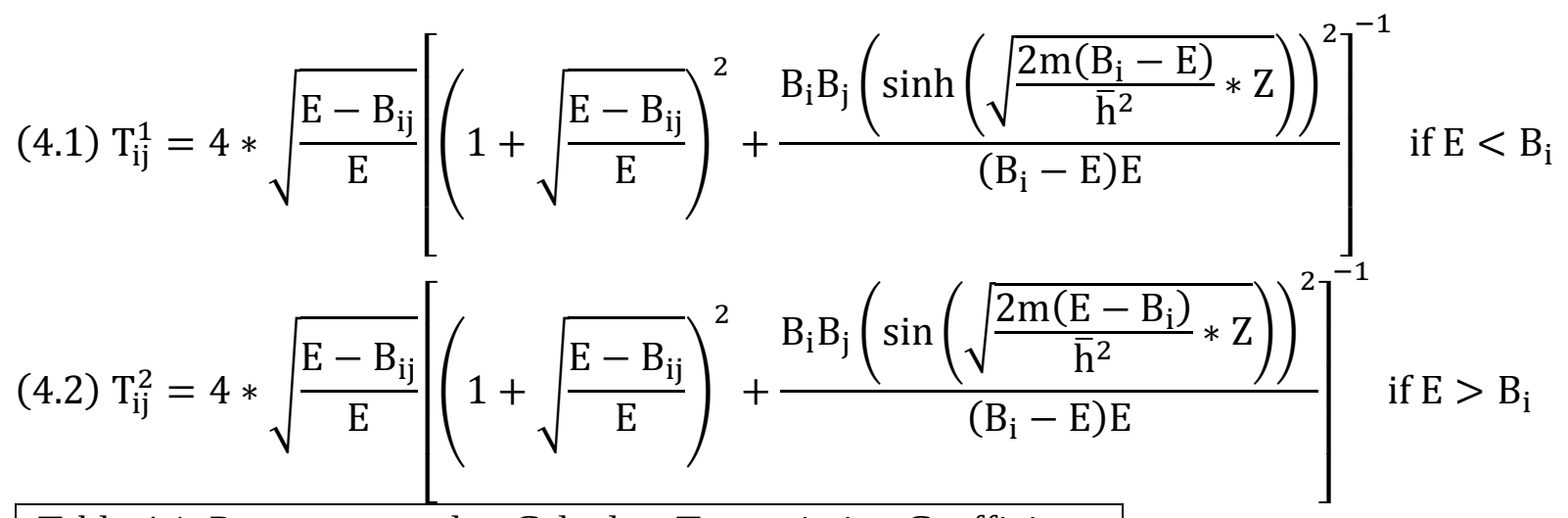

\begin{tabular}{|c|c|c|c|c|}
\hline \multicolumn{5}{|c|}{ Table 4.1: Parameter used to Calculate Transmission Coefficient: } \\
\hline $\mathrm{T}_{\mathrm{ij}}$ & $\mathrm{B}_{\mathrm{i}}$ & $\mathrm{B}_{\mathrm{j}}$ & $\mathrm{B}_{\mathrm{ij}}$ & $\mathrm{Z}$ \\
\hline $\mathrm{T}_{\mathrm{PP}}$ & $\mathrm{E}_{\mathrm{P}}(\mathrm{k})-\mathrm{E}_{\mathrm{SP}}$ & $\mathrm{E}_{\mathrm{P}}(\mathrm{k})-\mathrm{E}_{\mathrm{SP}}$ & 0 & $\mathrm{~A}$ \\
\hline $\mathrm{T}_{\mathrm{CC}}$ & $\mathrm{E}_{\mathrm{C}}(\mathrm{k})-\mathrm{E}_{\mathrm{SC}}$ & $\mathrm{E}_{\mathrm{C}}(\mathrm{k})-\mathrm{E}_{\mathrm{SC}}$ & 0 & $\mathrm{~A}$ \\
\hline $\mathrm{T}_{\mathrm{PC}}$ & $\mathrm{E}_{\mathrm{P}}(\mathrm{k})-\mathrm{E}_{\mathrm{S}}$ & $\mathrm{E}_{\mathrm{C}}(\mathrm{k})-\mathrm{E}_{\mathrm{S}}$ & $\mathrm{E}_{\mathrm{P}}(\mathrm{k})-\mathrm{E}_{\mathrm{C}}(\mathrm{k})$ & $\mathrm{a}$ \\
\hline $\mathrm{T}_{\mathrm{CP}}$ & $\mathrm{E}_{\mathrm{C}}(\mathrm{k})-\mathrm{E}_{\mathrm{S}}$ & $\mathrm{E}_{\mathrm{P}}(\mathrm{k})-\mathrm{E}_{\mathrm{S}}$ & $\mathrm{E}_{\mathrm{P}}(\mathrm{k})-\mathrm{E}_{\mathrm{C}}(\mathrm{k})$ & $\mathrm{a}$ \\
\hline
\end{tabular}

We will only briefly touch on this here but a more thorough background can be found in their 2004 paper and the corrections from 2010. ${ }^{12,51}$ The basic idea is to treat the competition between reaction and diffusion differently then what has been done in section 2.2.2. An atom adsorbed on a grain surface can visit several sites before being released into the gas phase or recombining when it encounters another atom. Currently in Nautilus this is treated with the reaction-diffusion 
competition probability described in section 2.2 .2 , but Cazaux et al. offer a different approach more suited for interactions between chemisorption and physisorption sites. The barriers used for molecular and atomic hydrogen and deuterium are outlined in Tables 1 and 2 in Cazaux et al. 2004 and the 2010 correction. ${ }^{12,51}$ The different barriers separating the two sites and defined in table 4.1 can be defined as follows. An atom ' $\mathrm{k}$ ' is defined and for physisorption the barrier width is defined by ' $a$ ', for chemisorption it's defined by ' $A$ '. Equation 4.1 is how tunneling from site ' $i$ ' to site ' $j$ ' is treated and equation 4.2 is how diffusion is treated. ' $Z$ ' is defined as the width of those barriers, $B_{i j}$ the energy of the barrier between site ' $i$ ' and ' $j$ ', $B_{i}$ is the energy of the barrier between two ' $i$ ' sites and $B_{j}$ is the energy of the barrier between two ' $j$ ' sites (see Figures 1-3 in Cazaux 2004). ${ }^{51}$ In their paper they assume only two distinct interactions between the atom and surface: physisorption and chemisorption. In the case of molecular hydrogen this approach may be valid but for more complex species that bind in defect sites it may be an oversimplification. However, clearly this is more valid then what's being considered in the current model. To extend their model to additional reactions some care would be needed in estimating barriers and we believe the best approach for this would be quantum calculations as defined above.

It's also worth noting how quantum tunneling is treated in this model. Tunneling was modelled using a square potential as in previous models (equation 2.24). We're also using a barrier width of $1 \mathrm{~A}^{\circ}$ which is what is used for physisorption sites. Tunneling is only important for light species like atomic hydrogen and deuterium and has been explored by Cazaux et al. to study the interaction between chemisorption and physisorption sites and how this effected hydrogen 
recombination. ${ }^{12,51}$ They found thermal hopping to dominate tunneling by $20-40 \mathrm{~K}$. The fact that the chemical barrier isn't well constrained in this model shouldn't lead to much error in the temperature regimes we're focused on.

Eventually additional reactions should be added, including things like the Haber Bosch process and oxo-process. 4, 8-9,

55 The current results presented were ran under constant physical conditions and standard conditions. To compare model results to observational abundances of a protoplanetary disk or hot core a few things should be considered. For instance, in the current model we're assuming the gas phase temperature is equal to the grain temperature. The grain temperature depends on the composition of the grain, the interstellar radiation field and the size of the grain. ${ }^{71}$ In Nautilus the grain temperature can be computed by assuming it's a function of UV flux and visual extinction. ${ }^{72}$ Physical conditions such as visual extinction, UV flux, hydrogen density etc. should also be adjusted accordingly. We've also assumed no X-ray radiation field which may be problematic when looking in a star forming region since the disk will be directly exposed to $\mathrm{X}$ rays and a UV field from the star. ${ }^{60}$ We assumed the unattenuated radiation field to be the same as previous models, this also may not be the case. It was found that both photodesorption and photodissociation were rather inefficient in these environments but making these adjustments this may change, especially for photodissication. In general, to extend this model to an astrophysical environment care needs to be taken in evaluating the physical parameters. 


\subsection{Conclusion:}

In this thesis chemisorption processes were added to a modified version of the three-phase gas-grain model developed by Ruaud et al. ${ }^{6,21}$ We added 316 reactions and 18 species, the final network had 11,800 reactions and 734 species. The hope was to explore how the gas phase abundances of alkanes would be affected by adding high temperature gas-grain chemistry and see if these results seemed likely based on our knowledge of astrophysical environments. The gasgrain formation route was only competitive with gas phase processes for the formation of methane, molecular hydrogen and the hydroxyl radical from 200-400 K. Most of the change in fractional abundances was due to enhancement or depletion of species in the gas phase. Although we're comfortable saying the gas phase abundance of molecular hydrogen and atomic oxygen is affected, we believe there's to many uncertainties in the barriers for different processes to say anything definitive about alkane formation. For these processes to be properly evaluated the barriers need to be constrained in a more efficient manner, possibly the quantum mechanical approach suggested earlier. It may also help to evaluate the chemisorption processes using a CTRW monte carlo technique since these barriers are sensitive to the surface structure.

\subsection{References:}

1. Herbst, E., Three milieux for interstellar chemistry: gas, dust, and ice. Phys Chem Chem Phys 2014, 16 (8), $3344-59$.

2. Drozdovskaya, M. N., Inextricable Ties between Chemical Complexity and Dynamics of Embedded Protostellar Regions. Ipskamp Printing: 2016. 
3. Monika E. Kress, A. G. G. M. T., The role of Fischer-Tropsch catalysis in solar nebula chemistry. Meteorites and Planetary Science 2001, 36, 75-91.

4. Joseph A. Nuth III, N. M. J., Steven Manning A self-perpetuating catalyst for the production of complex organic molecules in protostellar nebulae. Organic Matter in Space 2008, Proceedings IAU Symposium No. 251.

5. Garrod, W. W., Herbst, COMPLEX CHEMISTRY IN STAR-FORMING REGIONS: AN EXPANDED GAS-GRAIN WARM-UP CHEMICAL MODEL The Astrophysical Journal 2008, 682, 283-302.

6. Ruaud, M.; Wakelam, V.; Hersant, F., Gas and grain chemical composition in cold cores as predicted by the Nautilus three-phase model. Monthly Notices of the Royal Astronomical Society 2016, 459 (4), 3756-3767.

7. Duley, W., Interstellar Chemistry. Alden Press: 1984.

8. Hugh G.M. Hill, J. A. N., The Catalytic Potential of Cosmic Dust: Implications for Prebiotic Chemistry in the Solar Nebula and Other Protoplanetary Systems. Astrobiology 2003, 3 (2).

9. Nuth, J. A.; Johnson, N. M.; Ferguson, F. T.; Carayon, A., Gas/solid carbon branching ratios in surface-mediated reactions and the incorporation of carbonaceous material into planetesimals. Meteoritics \& Planetary Science 2016, 51 (7), 1310-1322.

10. Robert J. Gould, E. E. S., The Interstellar Abundance of the Hydrogen Molecule. 1. Basic Processes. ApJ 1963, 138.

11. Gould, S., The Interstellar Abundance of the Hydrogen Molecule. 1. Basic Processes. ApJ 1963.

12. Cazaux, S.; Tielens, A. G. G. M., ERRATUM: “H2FORMATION ON GRAIN SURFACES” (2004, ApJ, 604, 222). The Astrophysical Journal 2010, 715 (1), 698-699.

13. Garrod, R. T., A new modified-rate approach for gas-grain chemical simulations. Astronomy and Astrophysics 2008, 491 (1), $239-$ 251.

14. Garrod, R. T., Three-Dimensional, Off-Lattice Monte Carlo Kinetics Simulations of Interstellar Grain Chemistry and Ice Structure. The Astrophysical Journal 2013, 778 (2), 158.

15. Garrod, R. T.; Wakelam, V.; Herbst, E., Non-thermal desorption from interstellar dust grains via exothermic surface reactions. Astronomy and Astrophysics 2007, 467 (3), 1103-1115.

16. PE Curves \& Energetics of Adsorption. http://www.chem.qmul.ac.uk/surfaces/scc/scat2_4.htm.

17. Masel, R. I., Principles of Adsorption and Reaction on Solid Surfaces. Jon Wiley and Sons Inc.: 1996.

18. Christmann, K., Introduction to Surface Physical Chemistry. Springer: 1991.

19. Eric Herbst, T. J. M., The Chemistry of Cold Interstellar Cloud Cores. 2008.

20. Tatsuhiko I. Hasegawa, E. H., Models of Gas-Grain Chemistry in Dense Interstellar Clouds with Complex Organic Molecules. The Astrophysical Journal Supplement Series 1992, 82, 167-195.

21. Ruaud, M.; Loison, J. C.; Hickson, K. M.; Gratier, P.; Hersant, F.; Wakelam, V., Modelling complex organic molecules in dense regions: Eley-Rideal and complex induced reaction. Monthly Notices of the Royal Astronomical Society 2015, 447 (4), $4004-4017$.

22. Gomer, R., DIFFUSION OF ADSORBATES ON METAL-SURFACES. Rep. Prog. Phys. 1990, 53 (7), 917-1002.

23. SEKINE, S., SHIDO, YAMAMOTO,; IWASAWA, K., and MATSUI, An Experimental Study on Fischer-Tropsch Catalysis: Implications for impact phenomena and nebular chemistry. Meteorites and Planetary Science 2006, 41 (5), 715-729. 
24. Wakelam, S., Herbst, Troe, Geppert, Linnartz, Oberg, Roueff, Agundez, Pernot, Cuppen, Loison, Talbi, Reaction Networks for Interstellar Chemical Modelling: Improvements and Challenges. Space Science Review 2010, 156, 13-72.

25. Iqbal, W.; Acharyya, K.; Herbst, E., Kinetic Monte Carlo Studies of H2formation on Grain Surfaces over a Wide Temperature Range. The Astrophysical Journal 2012, 751 (1), 58.

26. Stantcheva, T.; Shematovich, V. I.; Herbst, E., On the master equation approach to diffusive grain-surface

chemistry: The H, O, CO system. Astronomy and Astrophysics 2002, 391 (3), 1069-1080.

27. Semenov, D.; Hersant, F.; Wakelam, V.; Dutrey, A.; Chapillon, E.; Guilloteau, S.; Henning, T.; Launhardt, R.; Piétu, V.; Schreyer, K., Chemistry in disks. Astronomy \& Astrophysics 2010, 522, A42.

28. $\quad$ Steinfield, F., Hase, Chemical Kinetics and Dynamics. Prentice Hall: 1999; Vol. 2.

29. Reboussin, L.; Wakelam, V.; Guilloteau, S.; Hersant, F., Grain-surface reactions in molecular clouds: the effect of cosmic rays and quantum tunnelling. Monthly Notices of the Royal Astronomical Society 2014, 440 (4), 3557-3567.

30. Tatsuhiko I. Hasegawa, E. H., Three-phase chemical models of dense interstellar clouds. Royal Astronomical Society 1993, (263), 589-606.

31. Su T, C., W.J., J Chem Phys 1982, 76 (5183).

32. Woon, D. E.; Herbst, E., Quantum Chemical Predictions of the Properties of Known and Postulated Neutral Interstellar Molecules. The Astrophysical Journal Supplement Series 2009, 185 (2), 273-288.

33. Wakelam Calculation of rate coefficients for ion-polar systems. http://kida.obs.ubordeaux1.fr/uploads/documents/ionpol_notice.pdf (accessed 9-28).

34. Harada, N.; Herbst, E.; Wakelam, V., A New Network for Higher-Temperature Gas-Phase Chemistry. I. A Preliminary Study of Accretion Disks in Active Galactic Nuclei. The Astrophysical Journal 2010, 721 (2), 1570-1578.

35. H.H. Lee, E. H., G. Pineau des Forets, and J. Le Bourlot, Photodissociation of H2 and CO and Time Dependent Chemistry in Inhomogenous Interstellar Clouds. Astronomy and Astrophysics 1996, 311, 690-707.

36. Visser, R.; van Dishoeck, E. F.; Black, J. H., The photodissociation and chemistry of CO isotopologues: applications to interstellar clouds and circumstellar disks. Astronomy \& Astrophysics 2009, 503 (2), 323-343.

37. David Hollenbach, C. F. M., Molecule Formation and Infrared Emmission in Fast Interstellar Schock. I. Physical Processes. The Astrophysical Journal Supplement Series 1979, 41, 555-592.

38. Garrod, R. T.; Pauly, T., On the Formation of Co2and Other Interstellar Ices. The Astrophysical Journal 2011,735 (1), 15.

39. Cacciatore, M.; Rutigliano, M., Dynamics of plasma-surface processes: E-R and L-H atom recombination reactions. Plasma Sources Science and Technology 2009, 18 (2).

40. Dulieu, F.; Congiu, E.; Noble, J.; Baouche, S.; Chaabouni, H.; Moudens, A.; Minissale, M.; Cazaux, S., How micron-sized dust particles determine the chemistry of our Universe. Sci Rep 2013, 3, 1338.

41. Fayolle, E. C.; Bertin, M.; Romanzin, C.; M Poderoso, H. A.; Philippe, L.; Michaut, X.; Jeseck, P.; Linnartz, H.; Öberg, K. I.; Fillion, J. H., Wavelength-dependent UV photodesorption of pure N2and O2ices. Astronomy \& Astrophysics 2013, 556, A122. 
42. Fayolle, E. C.; Öberg, K. I.; Cuppen, H. M.; Visser, R.; Linnartz, H., Laboratory H2O:CO2ice desorption data: entrapment dependencies and its parameterization with an extended three-phase model. Astronomy \& Astrophysics 2011, 529, A74.

43. Öberg, K. I.; Garrod, R. T.; van Dishoeck, E. F.; Linnartz, H., Formation rates of complex organics in UV irradiated $\mathrm{CH} 3 \mathrm{OH}$-rich ices. Astronomy and Astrophysics 2009, 504 (3), 891-913.

44. Oberg, F., Awad, Fraser, Schlemmer, Van Dischoeck, Linnartz, Photodesorption of CO Ice. The Astrophysical Journal 2007, 662, L23-L26.

45. Andersson, S.; van Dishoeck, E. F., Photodesorption of water ice. Astronomy and Astrophysics 2008, 491 (3), 907-916.

46. Shen, C. J.; Greenberg, J. M.; Schutte, W. A.; van Dishoeck, E. F., Cosmic ray induced explosive chemical desorption in dense clouds. Astronomy \& Astrophysics 2004, 415 (1), 203-215.

47. Muñoz Caro, G. M.; Jiménez-Escobar, A.; Martín-Gago, J. Á.; Rogero, C.; Atienza, C.; Puertas, S.; Sobrado, J. M.; Torres-Redondo, J., New results on thermal and photodesorption of $\mathrm{CO}$ ice using the novel InterStellar Astrochemistry Chamber (ISAC). Astronomy \& Astrophysics 2010, 522.

48. Wakelam, V.; Loison, J. C.; Herbst, E.; Pavone, B.; Bergeat, A.; Béroff, K.; Chabot, M.; Faure, A.; Galli, D.; Geppert, W. D.; Gerlich, D.; Gratier, P.; Harada, N.; Hickson, K. M.; Honvault, P.; Klippenstein, S. J.; Picard, S. D. L.; Nyman, G.; Ruaud, M.; Schlemmer, S.; Sims, I. R.; Talbi, D.; Tennyson, J.; Wester, R., The 2014 Kida Network for Interstellar Chemistry. The Astrophysical Journal Supplement Series 2015, $217(2), 20$.

49. S. Cazaux, A. G. G. M. T., Molecular Hydrogen Formation in the Interstellar Medium. The Astrophysical Journal 2002, 575, L29L32.

50. Cazaux, S.; Caselli, P.; Tielens, A. G. G. M.; Bourlot, J. L.; Walmsley, M., Molecular Hydrogen formation on grain surfaces. Journal of Physics: Conference Series 2005, 6, 155-160.

51. Cazaux, T., H2 Formation on Grain Surfaces The Astrophysical Journal 2004, 604, 222-237.

52. Gould, G., Salpeter, The Interstellar Abundance of the Hydrogen Molecule. 2. Galactic Abundance and Distribution. ApJ 1963.

53. Jelea, A.; Marinelli, F.; Ferro, Y.; Allouche, A.; Brosset, C., Quantum study of hydrogen-oxygen-graphite interactions. Carbon 2004, 42 (15), 3189-3198.

54. Edwin A. Bergin, G. J. M., David A. Neufeld, The Postshock Chemical Lifetimes of Outflow Tracers and a Possible New Mechanism to Produce Water Ice Mantles.pdf>. The Astrophysical Journal 1998, 499, 777-792.

55. Henry H. Storch, N. G., Robert B. Anderson, The Fischer-Tropsch and Related Syntheses. Jon Wiley and Sons Inc.: 1951.

56. T.J. Millar, C. M. L., E. Herbst, How Abundant are Complex Interstellar Molecules? Astronomy and Astrophysics 1987, 183, 109-

117.

57. Rodríguez, L. S.; Ruette, F.; Sánchez, M.; Mendoza, C., Interstellar catalysis: Formation of small molecules on a graphitic flake. Journal of Molecular Catalysis A: Chemical 2010, 316 (1-2), 16-22.

58. Calderón, L. A.; Chamorro, E.; Espinal, J. F., Mechanisms for homogeneous and heterogeneous formation of methane during the carbon-hydrogen reaction over zigzag edge sites. Carbon 2016, 102, 390-402. 
59. Liu, F.; Chu, W.; Sun, W.; Xue, Y.; Jiang, Q., A DFT study of methane activation on graphite surfaces with vacancy defects. Journal of Natural Gas Chemistry 2012, 21 (6), 708-712.

60. Bergin, E. A., The Chemical Evolution of Protoplanetary Disk. 2009.

61. Cleeves, O., Wilner, Huang, Andrews, Czekala, THE COUPLED PHYSICAL STRUCTURE OF GAS AND DUST IN THE IM Lup PROTOPLANETARY DISK. The Astrophysical Journal 2016.

62. Costanzo, F.; Silvestrelli, P. L.; Ancilotto, F., Physisorption, Diffusion, and Chemisorption Pathways of H2 Molecule on Graphene and on $(2,2)$ Carbon Nanotube by First Principles Calculations. J Chem Theory Comput 2012, 8 (4), 1288-94.

63. Petucci, J.; LeBlond, C.; Karimi, M.; Vidali, G., Diffusion, adsorption, and desorption of molecular hydrogen on graphene and in graphite. J Chem Phys 2013, 139 (4), 044706.

64. Morón, V.; Gamallo, P.; Sayós, R., DFT and kinetics study of O/O2 mixtures reacting over a graphite (0001) basal surface. Theoretical Chemistry Accounts 2010, 128 (4-6), 683-694.

65. Atkins, F., Molecular Quantum Mechanics. Oxford University Press: 2011; Vol. Fifth edition.

66. Woon, D. E., Ab-Initio Quantum Chemical Studies of Reactions in Astrophysical-Ices: 1 Aminolysis, Hydrolysis and Polymerization in $\mathrm{H} 2 \mathrm{CO} / \mathrm{NH} 3 / \mathrm{H} 2 \mathrm{O}$ Ices. Icarus 1999, 142, 550-556.

67. H. Bergeron, N. R., V. Sidis, M. Sizun, D. Teillet-Billy and F. Aguillon, OH Formation from $\mathrm{O}$ and $\mathrm{H}$ Atoms Physisorbed on a Graphitic Surface through the Langmuir-Hinshelwood Mechanism A Quasi-Classical Approach. Journal of Physical Chemistry 2008, 112, 11921-11930.

68. Hosseinnejad, T.; Abdullah Mirzaei, R.; Nazari, F.; Karimi-Jafari, M. H., Adsorption behavior of Co and C2H2 on the graphite basal surface: A quantum chemistry study. Journal of Structural Chemistry 2013, 54 (5), 850-856.

69. Strange, W. J., Carbon-carbon dioxide reaction Langmuir-Hinshelwood kinetics at intermediate pressures. Carbon 1976, 14, 345350.

70. Chang, Q.; Cuppen, H. M.; Herbst, E., Continuous-time random-walk simulation of H\$ \mathsf\{_\{2\}\}\$ formation on interstellar grains. Astronomy \& Astrophysics 2005, 434 (2), 599-611.

71. Iqbal, W.; Acharyya, K.; Herbst, E., H2formation in Diffuse Clouds: A New Kinetic Monte Carlo Study. The Astrophysical Journal 2014, $784(2), 139$.

72. Draine, B. T., Physics of the Interstellar and Intergalactic Medium. Princeton Series in Astrophysics: 2011.

73. Shustorovich, E., CHEMISORPTION THEORY - IN SEARCH OF THE ELEPHANT. Accounts Chem. Res. 1988, 21 (5), $183-189$.

74. S.C. Xu, S. I., D.G. Musaev, and M.C. Lin, Quantum Chemical Prediction of Reaction Pathways and Rate Constants for Dissociative Adsorption of COx and NOx on the Graphite (0001) Surface. Journal of Physical Chemistry 2006, 110, 21135-21144.

75. Yu, S. N.; Nejat, T. V., On the hydrogenation-dehydrogenation of graphene-layer-nanostructures: Relevance to the hydrogen onboard storage problem. International Journal of Physical Sciences 2015, 10 (2), 54-89. 


\subsection{Appendix:}

\subsection{Additional Equations Used:}

A carbonaceous grain was treated as a graphite surface and activation energies and dissociation energies were taken from the literature. However, in some instances a literature value wasn't available and a different approach was needed. To estimate activation energies an Evans-Polanyi Relation can be used: ${ }^{17}$

(A1) $\mathrm{E}_{\mathrm{A}}=\mathrm{E}_{0}+\mathrm{m}(\Delta \mathrm{H})$

The Evans-Polanyi relation states the barrier height of two similar reactions is inversely proportional to the enthalpy change of the reaction. It's a way to relate a third reaction to two similar reactions (assuming their energy barrier and heat of formation is known). The key to using this approach to estimate unknown activation energies is to make sure the reactions we're using have similar catalytic behavior to the dust grain being considered.

To estimate dissociation energies when a literature value isn't available the BOC-MP Model was used. ${ }^{17,73}$ Shustorovich developed a model in the $80^{\prime}$ s to predict dissociation energies for species that are chemisorbed to a surface. ${ }^{73}$ This model is based on bond order conservation (BOC) and makes use of Morse potentials (MP). Since we're dealing with adsorbates that are primarily chemisorbed to the surface this is the preferred method for predicting dissociation energies. Consider a simple reaction where an atom ' $\mathrm{A}$ ', is transferred from an adsorbed B-A molecule to a surface ' $\mathrm{S}$ ': 
(A2) $\mathrm{B}-\mathrm{A}_{\mathrm{ads}}+\mathrm{S} \rightarrow \mathrm{B}_{\mathrm{ads}}+\mathrm{A}_{\mathrm{ads}}$

It's assumed that all of the atomic interactions in this reaction can be modelled by a Morse potential. For example, the B-A potential can be modelled as follows:

(A3) $V_{B A}\left(r_{B A}\right)=D_{B A}\left[\left(\exp \left[-\alpha_{B A}\left(r_{B A}-r_{B A}^{o}\right)\right]-1\right)^{2}-1\right]$

where $D_{B A}$ is the bond dissociation energy of the $B-A$ bond, $r_{B A}$ is the length of the $B-A$ bond, $r_{B A}^{o}$ is the equilibrium bond length and $\alpha_{B A}$ is related to the force constant for the B-A bond. The B-surface and A-surface bonds are also assumed to follow a Morse potential:

(A4) $V_{B S}\left(r_{B S}\right)=Q_{B}\left[\left(\exp \left[-\alpha_{B S}\left(r_{B S}-r_{B S}^{o}\right)\right]-1\right)^{2}-1\right]$

(A5) $\mathrm{V}_{\mathrm{AS}}\left(\mathrm{r}_{\mathrm{AS}}\right)=\mathrm{Q}_{\mathrm{A}}\left[\left(\exp \left[-\alpha_{\mathrm{AS}}\left(\mathrm{r}_{\mathrm{AS}}-\mathrm{r}_{\mathrm{AS}}^{\mathrm{o}}\right)\right]-1\right)^{2}-1\right]$

where $\mathrm{V}_{\mathrm{BS}}$ and $\mathrm{V}_{\mathrm{AS}}$ are the energies of the B-S and $\mathrm{A}-\mathrm{S}$ bonds, $\mathrm{r}_{\mathrm{BS}}$ and $\mathrm{r}_{\mathrm{AS}}$ are the bond lengths, $\mathrm{r}_{\mathrm{BS}}^{\mathrm{o}}$ and $\mathrm{r}_{\mathrm{AS}}^{\mathrm{o}}$ are equilibrium bond lengths, and $\mathrm{Q}_{\mathrm{B}}$ and $\mathrm{Q}_{\mathrm{A}}$ are the strengths of the $\mathrm{B}$-surface and $\mathrm{A}$-surface bonds.

Shustrovich also makes the assumption that bond order is conserved in reaction A2. After doing considerable algebra which is described in more detail in Shustrovich's original paper, ${ }^{73}$ we arrive at an expression for the dissociation energy: 
(A6) $\mathrm{E}_{\mathrm{A}}^{\mathrm{BA}_{\mathrm{ads}} \rightarrow \mathrm{B}_{\mathrm{ads}}+\mathrm{A}_{\mathrm{ads}}}=\frac{1}{2}\left(\mathrm{D}_{\mathrm{BA}}+\frac{\mathrm{Q}_{\mathrm{A}} \mathrm{Q}_{\mathrm{B}}}{\mathrm{Q}_{\mathrm{A}}+\mathrm{Q}_{\mathrm{B}}}+\mathrm{Q}_{\mathrm{BA}}-\mathrm{Q}_{\mathrm{A}}-\mathrm{Q}_{\mathrm{B}}\right)$

where $\mathrm{Q}_{\mathrm{BA}}$ is the heat of adsorption of the $\mathrm{BA}$ molecule and every other term is as previously defined. The heat of reaction can be defined as follows:

(A7) $\Delta \mathrm{H}_{\mathrm{r}}=\mathrm{D}_{\mathrm{BA}}+\mathrm{Q}_{\mathrm{BA}}-\mathrm{Q}_{\mathrm{A}}-\mathrm{Q}_{\mathrm{B}}$

Therefor equation $\mathrm{A} 6$ is equivalent to a Polanyi relationship:

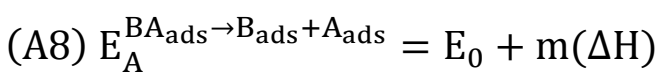

Assuming $\Delta \mathrm{G}_{\mathrm{r}}$ is small $\mathrm{m}$ should be close to $.5 \cdot{ }^{17} \Delta \mathrm{H}$ is heat of reaction which can either be found in literature or estimated using the Benziger approach, and $\mathrm{E}_{0}$ is defined as follows:

(A9) $\mathrm{E}_{0}=\frac{1}{2} * \frac{\mathrm{Q}_{\mathrm{A}} \mathrm{Q}_{\mathrm{B}}}{\mathrm{Q}_{\mathrm{A}}+\mathrm{Q}_{\mathrm{B}}}$

where $\mathrm{Q}_{\mathrm{A}}$ and $\mathrm{Q}_{\mathrm{B}}$ were defined previously.

Finding literature values for heat of adsorption proved to be difficult. These were needed for the BOC-MP method and for reactive desorption as defined previously in section 2. Benziger has a simple approach to estimate the heat of adsorption of reactive fragments. ${ }^{17}$ The basic idea is to add up the energies of all the bonds in the molecule to estimate a 
heat of adsorption. Consider an adsorbed fragment $\mathrm{RX}_{\mathrm{ads}}$, using Benziger's approach one can estimate the heat of formation of the adsorbed species as follows:

$\left(\right.$ A10) $\Delta \mathrm{H}_{\mathrm{f}}\left(\mathrm{RX}_{\mathrm{ads}}\right)=\Delta \mathrm{H}_{\mathrm{f}}(\mathrm{RX} \cdot)+\frac{\mathrm{n}_{\mathrm{sx}}}{\mathrm{n}_{\mathrm{x}}} \mathrm{D}\left(\mathrm{S}-\mathrm{RX}_{\mathrm{ads}}\right)$

where $\Delta \mathrm{H}_{\mathrm{f}}(\mathrm{RX} \cdot)$ is the heat of formation of the $\mathrm{RX} \cdot$ radical in the gas phase, $\mathrm{D}(\mathrm{S}-\mathrm{RX}$ ads $)$ is the strength of the $\mathrm{S}-\mathrm{RX}$ ads bond (where $\mathrm{S}$ is the surface), $\mathrm{n}_{\mathrm{Sx}}$ is the number of bonds between the surface and adsorbed molecule $\mathrm{RX}_{\mathrm{ads}}$, and $\mathrm{n}_{\mathrm{x}}$ is the number of bonds to atom $X$.

\subsection{Additional Figures and Tables:}

\begin{tabular}{|ll|c|}
\hline \multicolumn{3}{|l|}{ Table A1: Full list of reactions added to network: } \\
\hline \multicolumn{3}{|l|}{ reactants } \\
products
\end{tabular}




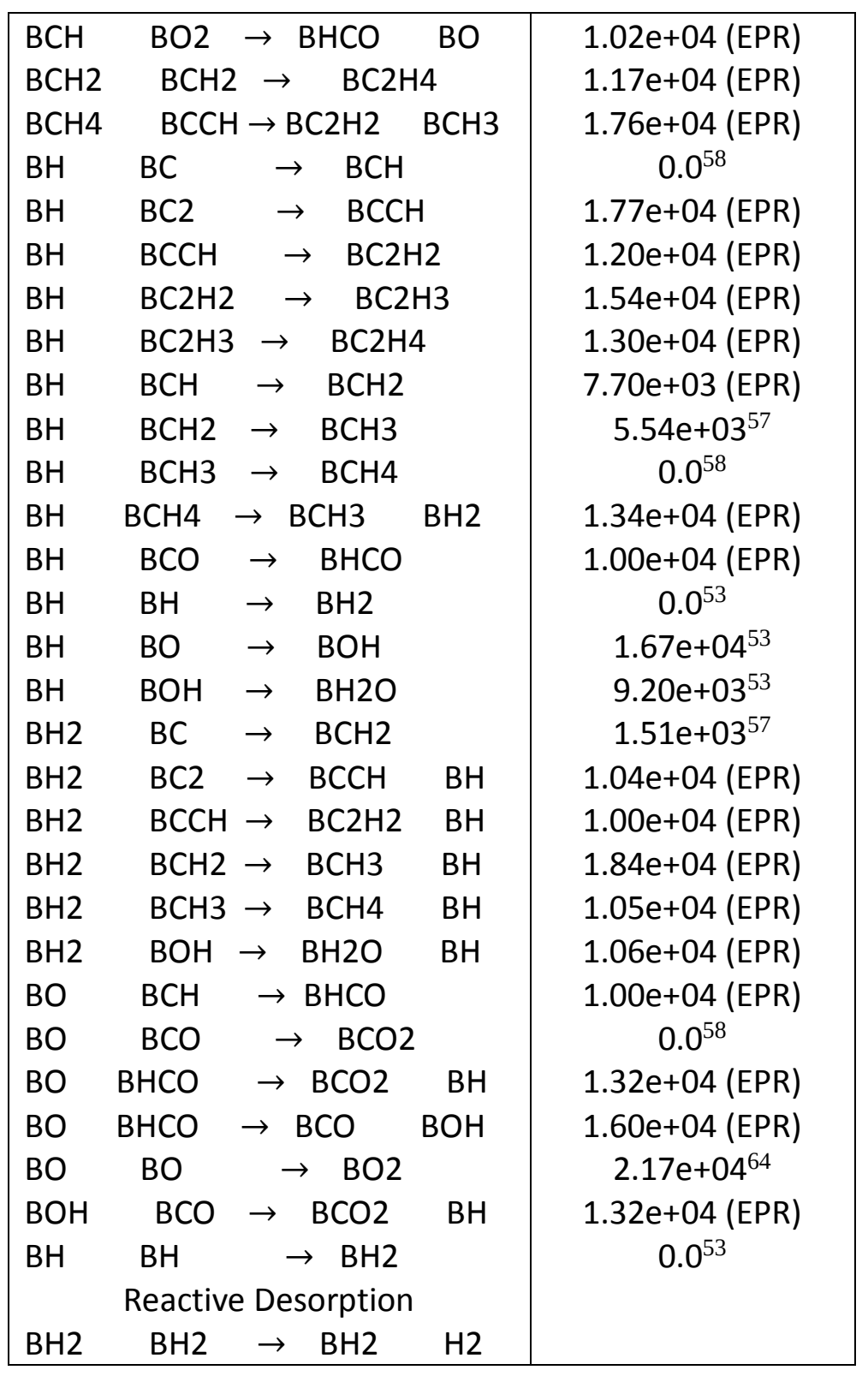




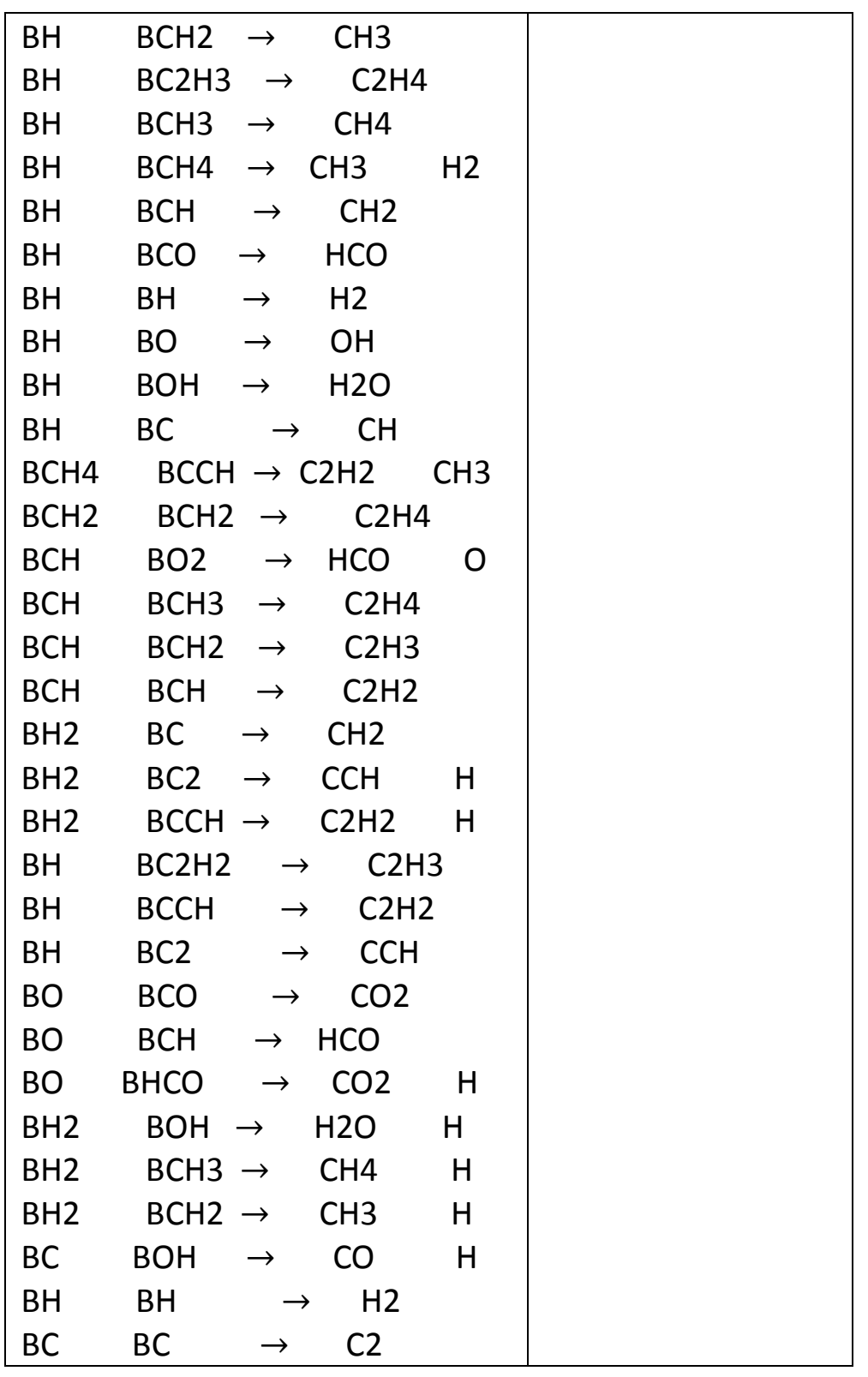




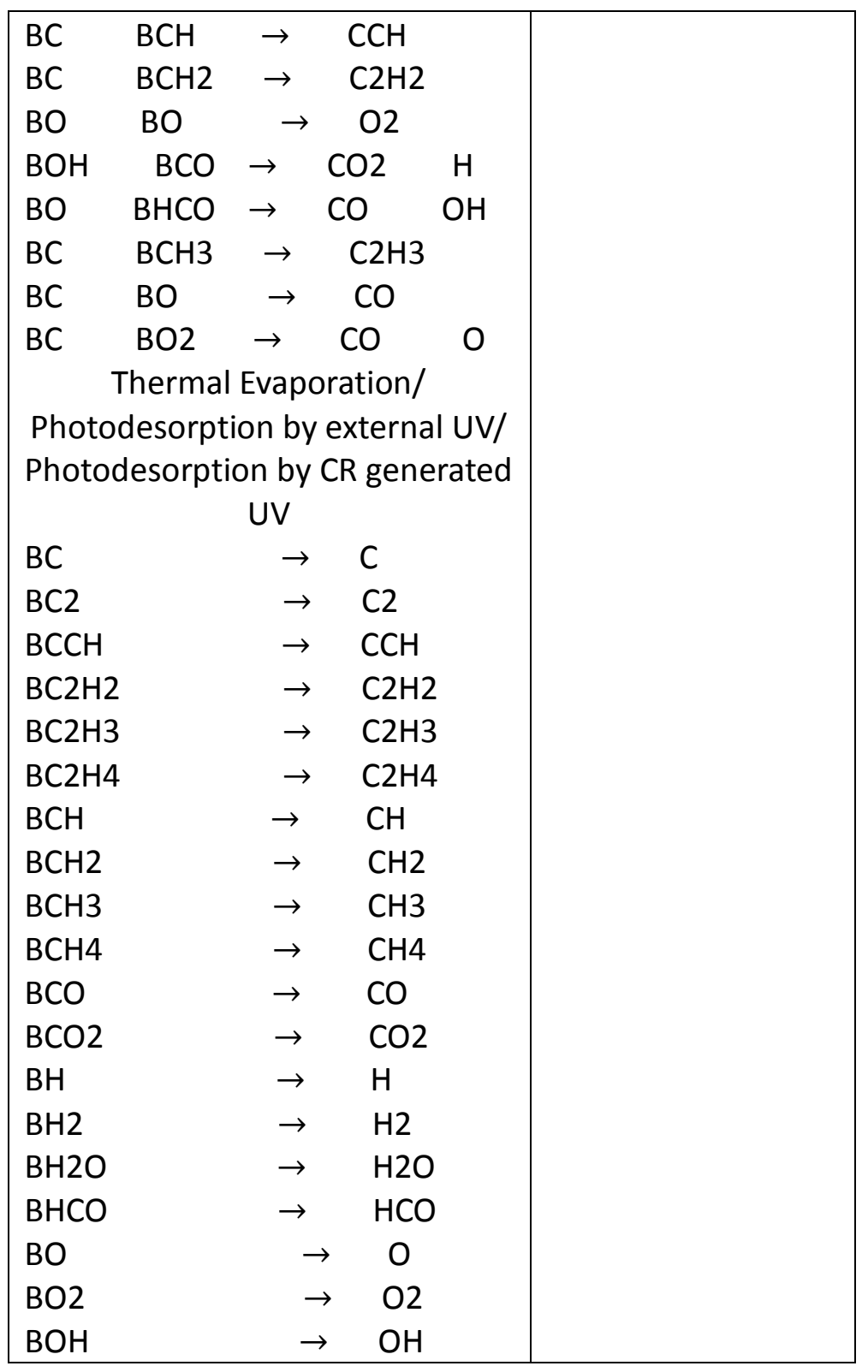




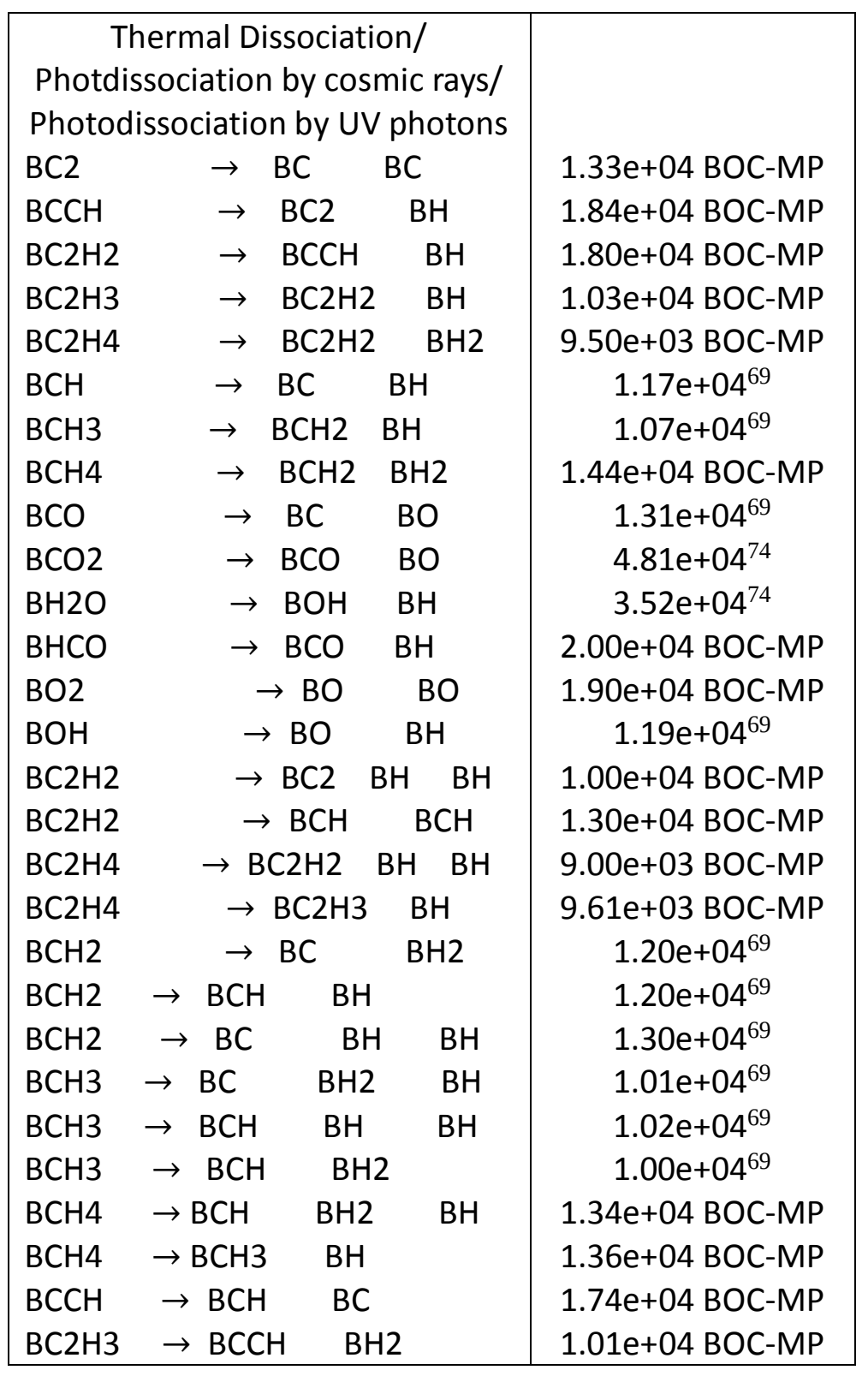




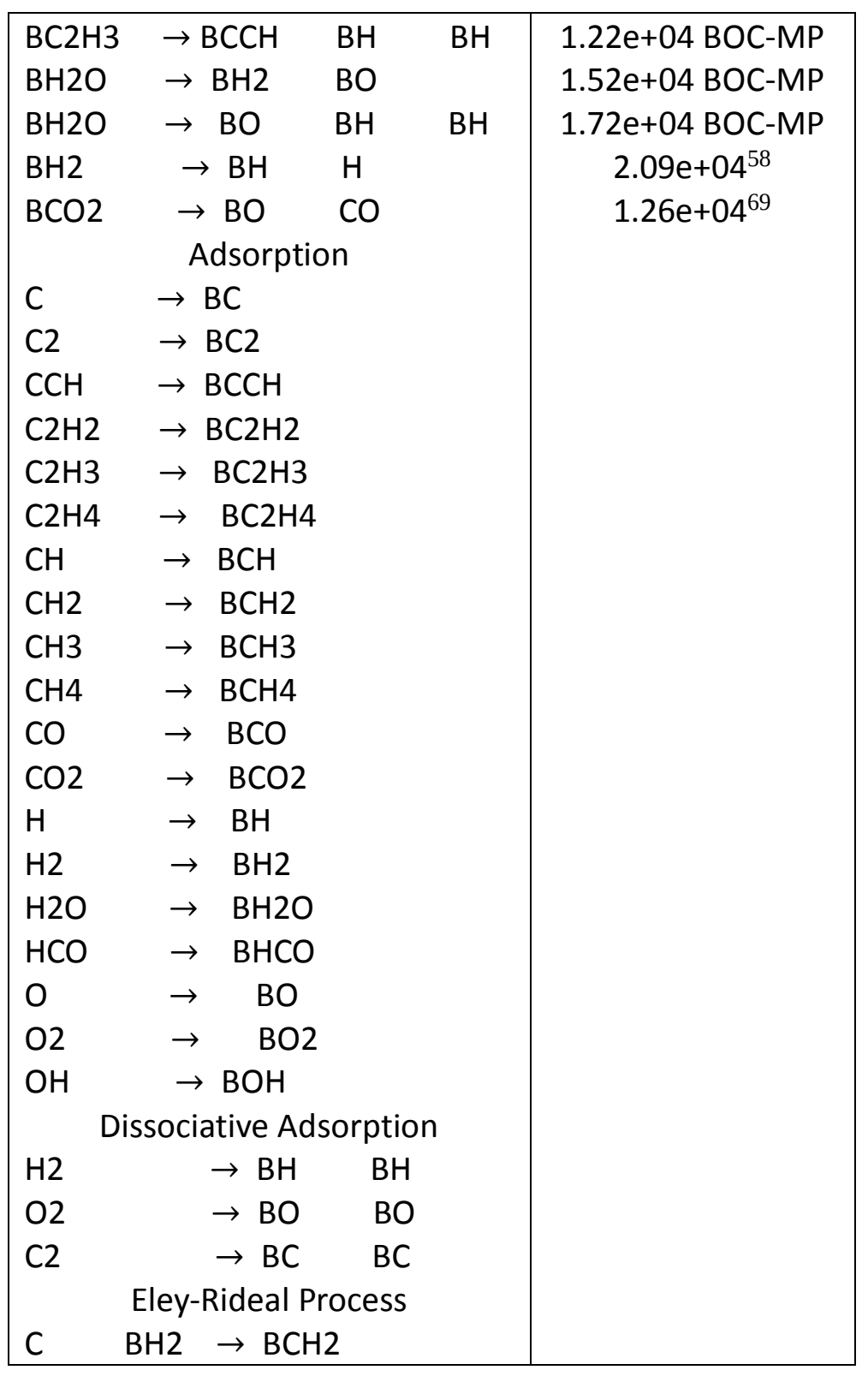




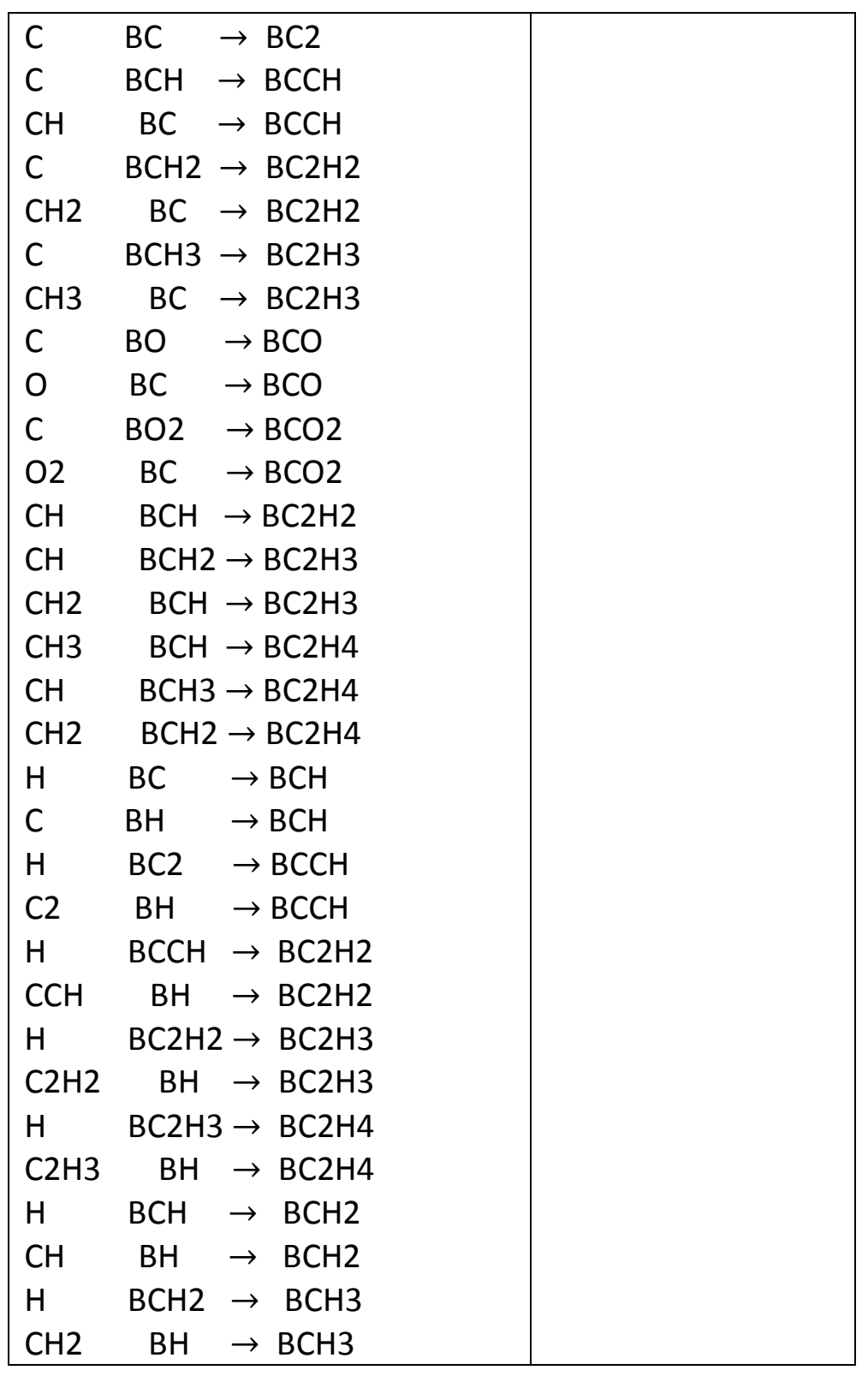




\begin{tabular}{|lcl|l|}
\hline $\mathrm{H}$ & $\mathrm{BCH} 3$ & $\rightarrow \mathrm{BCH} 4$ & \\
$\mathrm{CH} 3$ & $\mathrm{BH}$ & $\rightarrow \mathrm{BCH} 4$ & \\
$\mathrm{H}$ & $\mathrm{BCO}$ & $\rightarrow \mathrm{BHCO}$ & \\
$\mathrm{CO}$ & $\mathrm{BH}$ & $\rightarrow \mathrm{BHCO}$ & \\
$\mathrm{H}$ & $\mathrm{BO}$ & $\rightarrow \mathrm{BOH}$ & \\
$\mathrm{O}$ & $\mathrm{BH}$ & $\rightarrow \mathrm{BOH}$ & \\
$\mathrm{H}$ & $\mathrm{BOH}$ & $\rightarrow \mathrm{BH} 2 \mathrm{O}$ & \\
$\mathrm{OH}$ & $\mathrm{BH}$ & $\rightarrow \mathrm{BH} 2 \mathrm{O}$ & \\
$\mathrm{H} 2$ & $\mathrm{BC}$ & $\rightarrow \mathrm{BCH} 2$ & \\
$\mathrm{C}$ & $\mathrm{BH} 2$ & $\rightarrow \mathrm{BCH} 2$ & \\
$\mathrm{O}$ & $\mathrm{BCH}$ & $\rightarrow \mathrm{BHCO}$ \\
$\mathrm{CH}$ & $\mathrm{BO}$ & $\rightarrow \mathrm{BHCO}$ & \\
$\mathrm{O}$ & $\mathrm{BCO}$ & $\rightarrow \mathrm{BCO} 2$ & \\
$\mathrm{CO}$ & $\mathrm{BO}$ & $\rightarrow \mathrm{BCO} 2$ \\
$\mathrm{O}$ & $\mathrm{BO}$ & $\rightarrow \mathrm{BO} 2$ \\
$\mathrm{H}$ & $\mathrm{BH}$ & $\rightarrow \mathrm{BH} 2$ & \\
\hline
\end{tabular}

* In Table A1 there's two processes that will have barriers, grain surface reactions and the new thermal dissociation process. The carbon grain is assumed to have similar catalytic properties to a graphite surface so we attempted to use literature values. This wasn't always possible so for activation energies an Evans-Polanyi relation was used to estimate barriers, and for dissociation the BOC-MP Method. ${ }^{17,73}$

\begin{tabular}{|c|c|c|c|}
\hline \multicolumn{4}{|c|}{ Table A2: Grain species added to network and } \\
\multicolumn{3}{|c|}{ corresponding desorption energies and enthalpy of } \\
\hline $\begin{array}{l}\text { Species } \\
\text { added: }\end{array}$ & $\mathrm{E}_{\text {Des }}(\mathrm{K}):$ & $\Delta \mathrm{H}_{\mathrm{f}}\left(\frac{\mathrm{kcal}}{\mathrm{mol}}\right)$ & $\begin{array}{c}\text { Comments } \\
\left(\mathrm{E}_{\text {Des }} / \Delta \mathrm{H}_{\mathrm{f}}\right)\end{array}$ \\
\hline BC & 57231 & 285.01 & $\begin{array}{c}\text { Nechaev et al. } \\
(2015)^{75} / \\
\text { Benziger's }\end{array}$ \\
& & & $\begin{array}{c}\text { approach (BA) } \\
\text { (B) }\end{array}$ \\
\hline BC2 & 1600 & 196.60 & Cacciatore 2009 \\
\hline
\end{tabular}




\begin{tabular}{|c|c|c|c|}
\hline & & & $\begin{array}{c}(\mathrm{C} 9)^{39}, \text { Physisorbed } \\
\text { values (PV) }\end{array}$ \\
\hline $\mathrm{BCCH}$ & 21370 & 135.23 & $\mathrm{BA}$ \\
\hline $\mathrm{BC} 2 \mathrm{H} 2$ & 25870 & 71.32 & $\mathrm{BA}$ \\
\hline BO2 & 1393 & 0.00 & Moron $2010^{64}, \mathrm{PV}$ \\
\hline $\mathrm{BO}$ & 29046 & 117.27 & $\begin{array}{c}\text { Jelea } 2004(\mathrm{~J} 4)^{53} \\
\text { BA }\end{array}$ \\
\hline $\mathrm{BC} 2 \mathrm{H} 3$ & 30370 & 91.12 & $\mathrm{BA}$ \\
\hline $\mathrm{BC} 2 \mathrm{H} 4$ & 34870 & 35.64 & $\mathrm{BA}$ \\
\hline $\mathrm{BCH}$ & 71231 & 212.77 & $\mathrm{BA}$ \\
\hline $\mathrm{BCH} 2$ & 85231 & 148.80 & BA \\
\hline $\mathrm{BOH}$ & 6074 & 15.35 & $\mathrm{~J} 4^{53}, \mathrm{BA}$ \\
\hline $\mathrm{BH} 2 \mathrm{O}$ & 5700 & -57.10 & $\mathrm{~J} 4^{53}, \mathrm{PV}$ \\
\hline $\mathrm{BCH} 3$ & 10259 & 39.92 & $\begin{array}{c}\text { Calderon } 2016 \\
(\mathrm{C} 16)^{58}, \mathrm{BA}\end{array}$ \\
\hline $\mathrm{BCH} 4$ & 13108 & -15.90 & $\begin{array}{c}\text { Liu } 2012(\mathrm{~L} 12)^{59}, \\
\text { BA }\end{array}$ \\
\hline $\mathrm{BCO}$ & 40258 & 13.58 & $\begin{array}{c}\text { Strange } 1976^{69}, \\
\text { BA }\end{array}$ \\
\hline $\mathrm{BCO} 2$ & 19604 & -93.96 & $\mathrm{~L} 12^{59}, \mathrm{BA}$ \\
\hline $\mathrm{BH}$ & 14000 & 79.92 & Cazaux $2004^{51}, \mathrm{BA}$ \\
\hline $\mathrm{BH} 2$ & 440 & 0.00 & $(\mathrm{C} 9)^{39}, \mathrm{PV}$ \\
\hline
\end{tabular}

*In Table A2, in the comments section, you'll find references for where the desorption energies were taken from. The carbonaceous grain was assumed to behave similar to graphite and in some circumstances a literature value wasn't available, in which case the binding energy was treated as a free parameter. Enthalpy of formation was estimated using Benziger's Approach. ${ }^{17}$ Some species, particularly closed shell molecules like molecular hydrogen, won't chemisorb on a grain surface (PV stands for physisorbed value and previous values were used for both the binding energy and enthalpy of formation). 


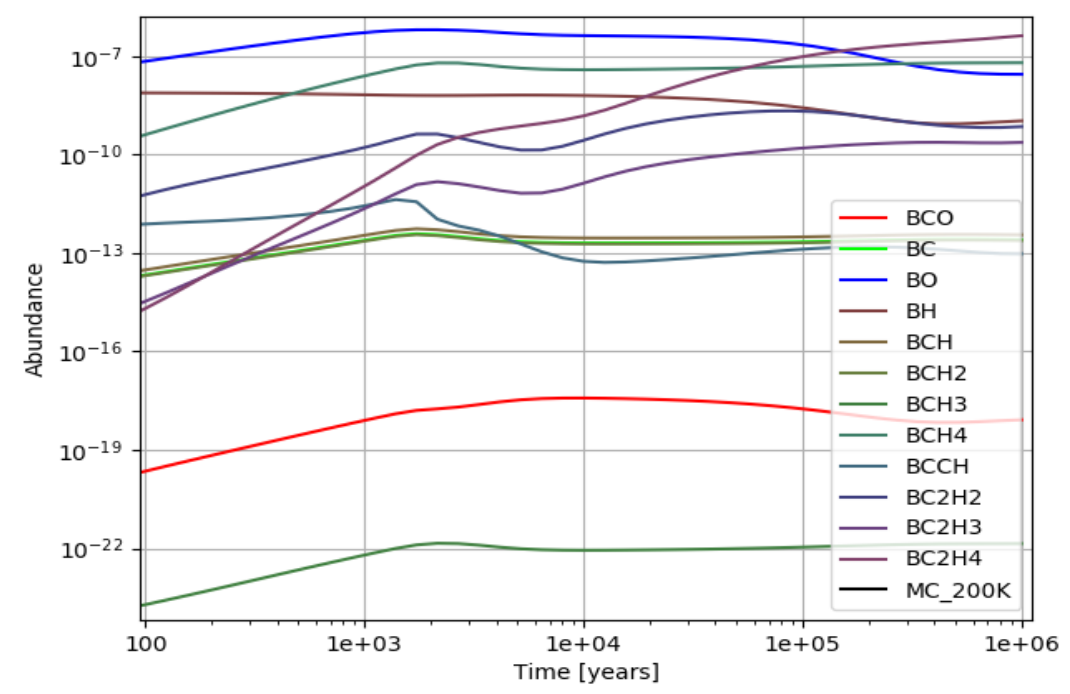

Figure A.1: Fractional abundance of $\mathrm{BCO}, \mathrm{BC}, \mathrm{BO}, \mathrm{BH}, \mathrm{BCCH}, \mathrm{BCH}_{2}, \mathrm{BCH}_{3}, \mathrm{BCH}_{4}, \mathrm{BC}_{2} \mathrm{H}_{2}, \mathrm{BC}_{2} \mathrm{H}_{3}$ and $\mathrm{BC}_{2} \mathrm{H}_{4}$ with respect to $\mathrm{n}_{\mathrm{H}}$ as a function of time for a molecular cloud ran under standard conditions at $200 \mathrm{~K}$. 


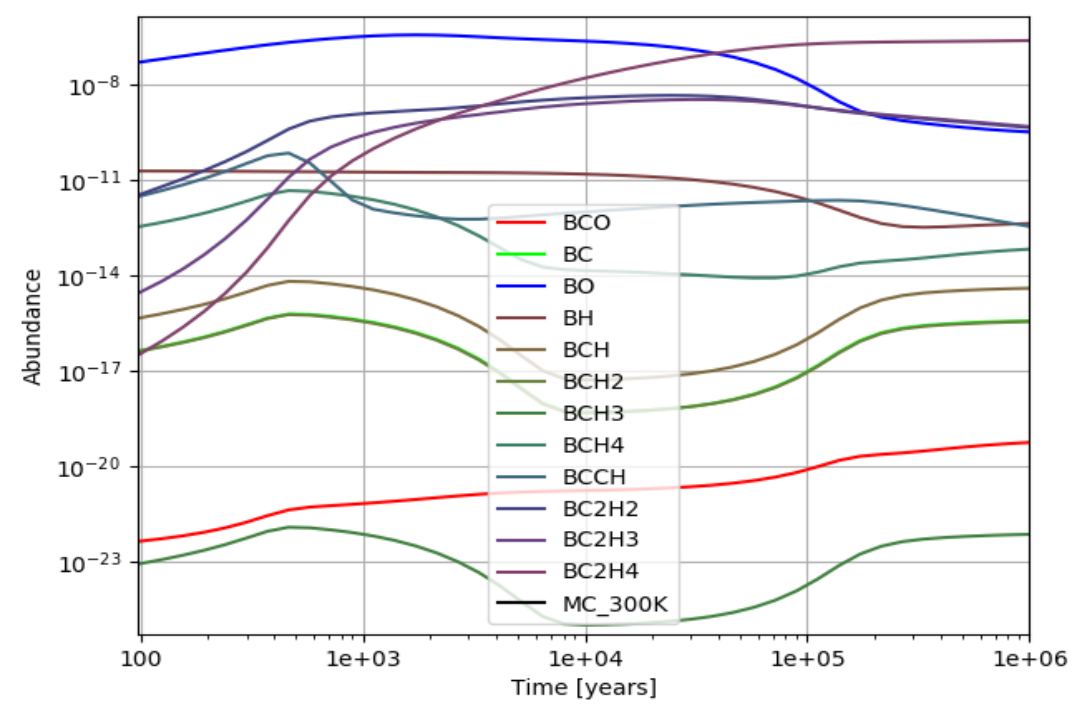

Figure A.2: Fractional abundance of $\mathrm{BCO}, \mathrm{BC}, \mathrm{BO}, \mathrm{BH}, \mathrm{BCCH}, \mathrm{BCH}_{2}, \mathrm{BCH}_{3}, \mathrm{BCH}_{4}, \mathrm{BC}_{2} \mathrm{H}_{2}, \mathrm{BC}_{2} \mathrm{H}_{3}$ and $\mathrm{BC}_{2} \mathrm{H}_{4}$ with respect to $\mathrm{n}_{\mathrm{H}}$ as a function of time for a molecular cloud ran under standard conditions at $300 \mathrm{~K}$. 


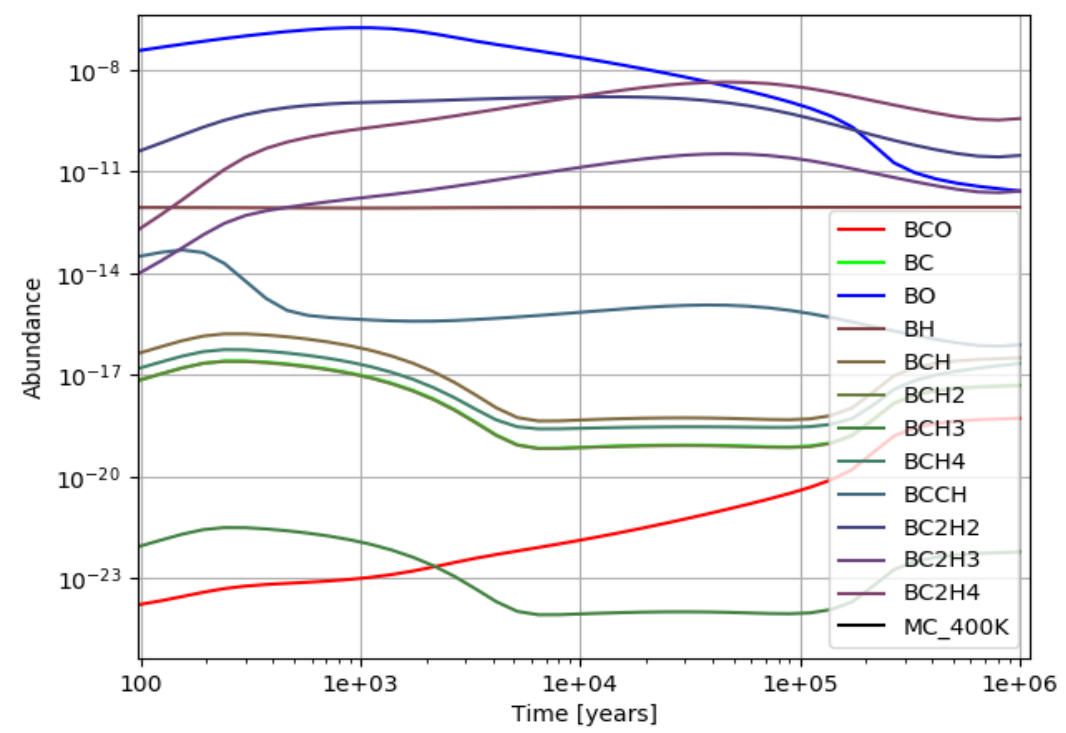

Figure A.3: Fractional abundance of $\mathrm{BCO}, \mathrm{BC}, \mathrm{BO}, \mathrm{BH}, \mathrm{BCCH}, \mathrm{BCH}_{2}, \mathrm{BCH}_{3}, \mathrm{BCH}_{4}, \mathrm{BC}_{2} \mathrm{H}_{2}, \mathrm{BC}_{2} \mathrm{H}_{3}$ and $\mathrm{BC}_{2} \mathrm{H}_{4}$ with respect to $\mathrm{n}_{\mathrm{H}}$ as a function of time for a molecular cloud ran under standard conditions at $400 \mathrm{~K}$. 


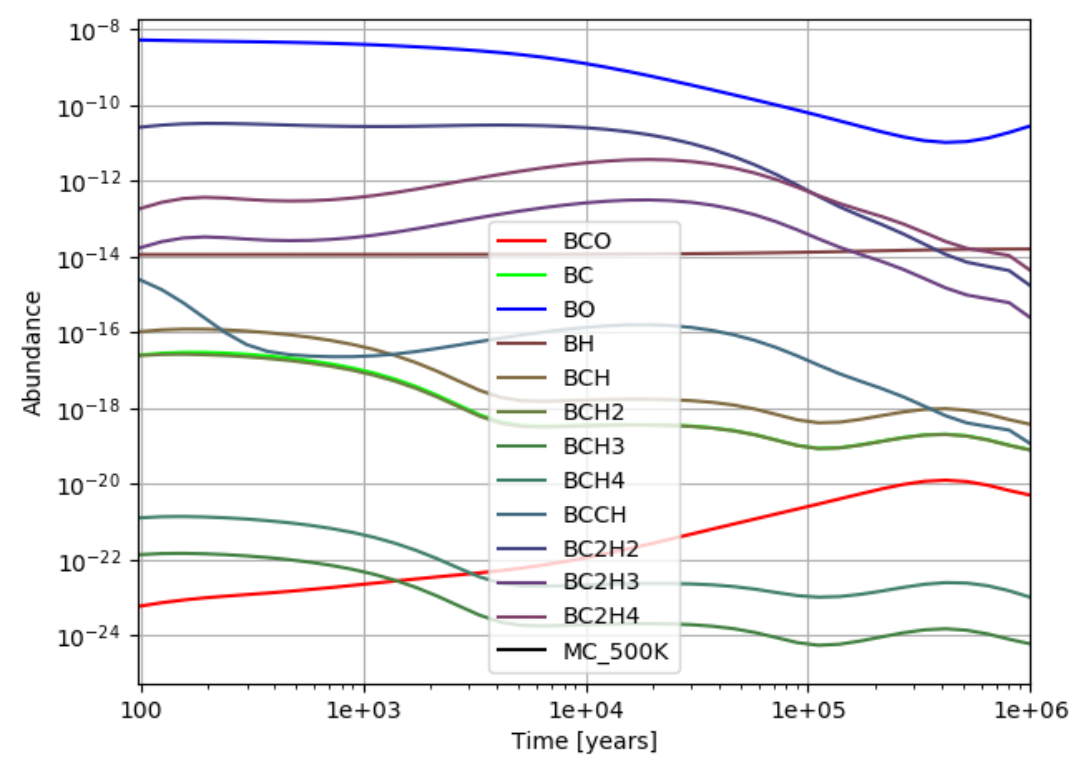

Figure A.4: Fractional abundance of $\mathrm{BCO}, \mathrm{BC}, \mathrm{BO}, \mathrm{BH}, \mathrm{BCCH}, \mathrm{BCH}_{2}, \mathrm{BCH}_{3}, \mathrm{BCH}_{4}, \mathrm{BC}_{2} \mathrm{H}_{2}, \mathrm{BC}_{2} \mathrm{H}_{3}$ and $\mathrm{BC}_{2} \mathrm{H}_{4}$ with respect to $\mathrm{n}_{\mathrm{H}}$ as a function of time for a molecular cloud ran under standard conditions at $500 \mathrm{~K}$. 


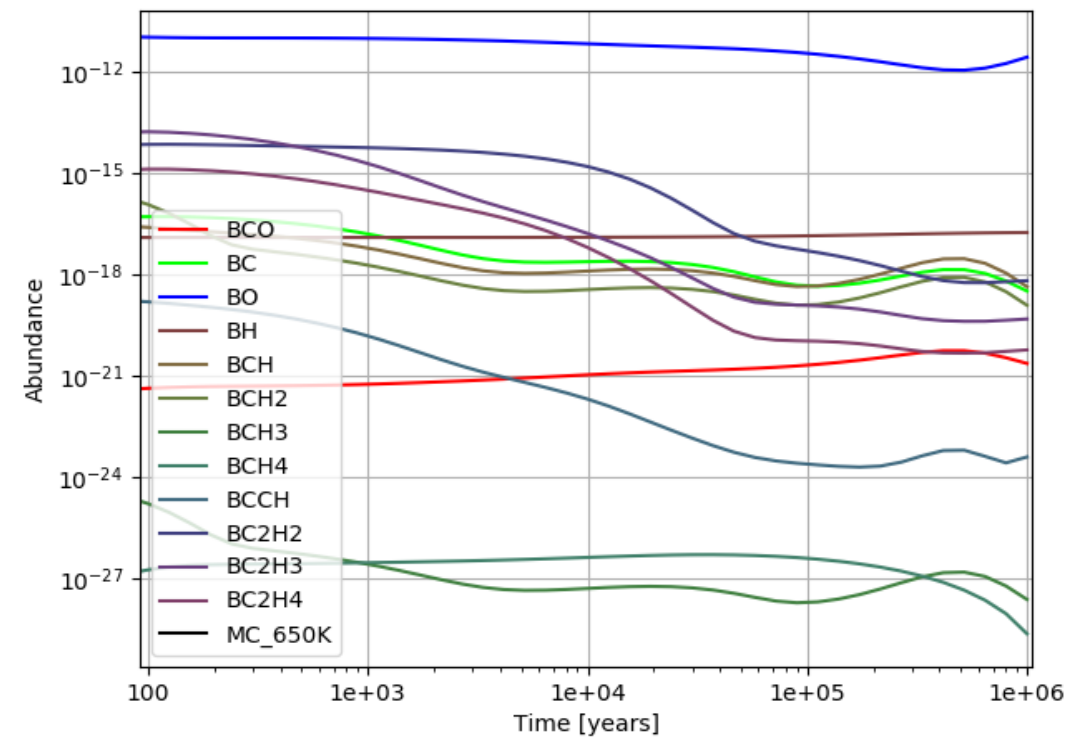

Figure A.5: Fractional abundance of $\mathrm{BCO}, \mathrm{BC}, \mathrm{BO}, \mathrm{BH}, \mathrm{BCCH}, \mathrm{BCH}_{2}, \mathrm{BCH}_{3}, \mathrm{BCH}_{4}, \mathrm{BC}_{2} \mathrm{H}_{2}, \mathrm{BC}_{2} \mathrm{H}_{3}$ and $\mathrm{BC}_{2} \mathrm{H}_{4}$ with respect to $\mathrm{n}_{\mathrm{H}}$ as a function of time for a molecular cloud ran under standard conditions at $650 \mathrm{~K}$. 


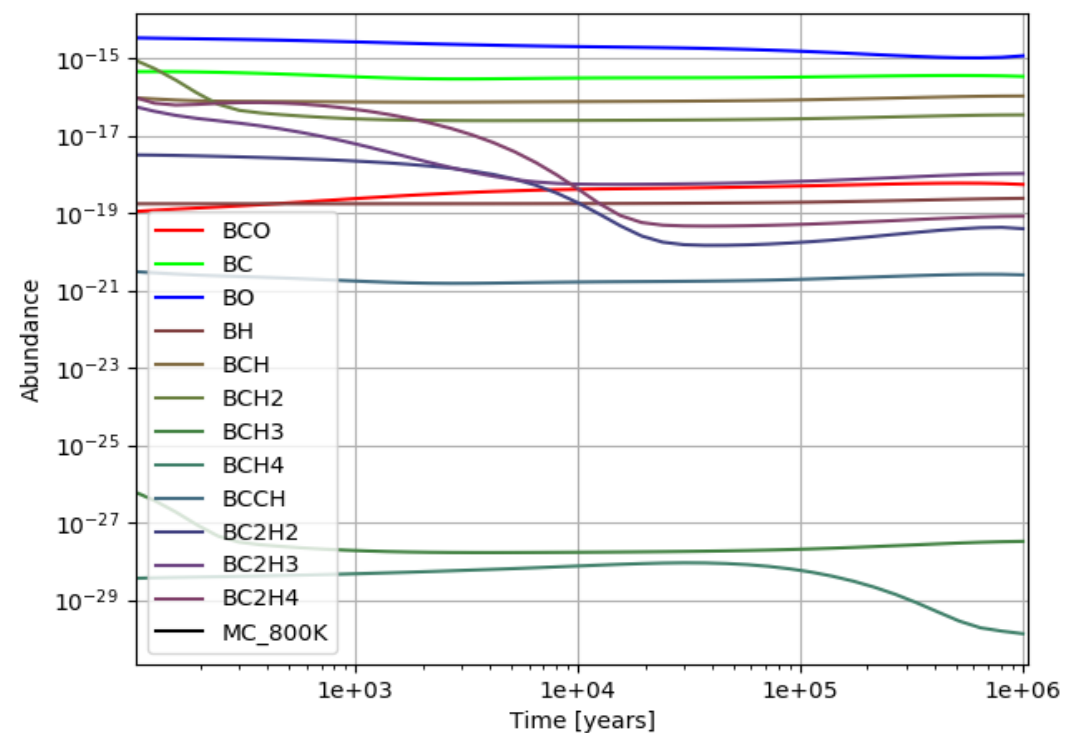

Figure A.6: Fractional abundance of $\mathrm{BCO}, \mathrm{BC}, \mathrm{BO}, \mathrm{BH}, \mathrm{BCCH}, \mathrm{BCH}_{2}, \mathrm{BCH}_{3}, \mathrm{BCH}_{4}, \mathrm{BC}_{2} \mathrm{H}_{2}, \mathrm{BC}_{2} \mathrm{H}_{3}$ and $\mathrm{BC}_{2} \mathrm{H}_{4}$ with respect to $\mathrm{n}_{\mathrm{H}}$ as a function of time for a molecular cloud ran under standard conditions at $800 \mathrm{~K}$. 